\title{
INTRINSIC DIFFERENTIABILITY AND INTRINSIC REGULAR SURFACES IN CARNOT GROUPS
}

\author{
DANIELA DI DONATO
}

\begin{abstract}
A Carnot group $\mathbb{G}$ is a connected, simply connected, nilpotent Lie group with stratified Lie algebra. Intrinsic regular surfaces in Carnot groups play the same role as $\mathbb{C}^{1}$ surfaces in Euclidean spaces. As in Euclidean spaces, intrinsic regular surfaces can be locally defined in different ways: e.g. as non critical level sets or as continuously intrinsic differentiable graphs. The equivalence of these natural definitions is the problem that we are studying. Precisely our aim is to generalize the results on 2] valid in Heisenberg groups to the more general setting of Carnot groups.
\end{abstract}

\section{INTRODUCTION}

In the last years a systematic attempt to develop a good notion of rectifiable sets in metric space and in particular inside Carnot groups, has become the object of many studies. For a general theory of rectifiable sets in Euclidean spaces one can see [9, 8, 23] while a general theory in metric spaces can be found in [1].

Rectifiable sets are classically defined as contained in the countable union of $\mathbb{C}^{1}$ submanifolds. In this paper we focus our attention on the natural notion of $\mathbb{C}^{1}$ surface, inside a special class of metric spaces i.e. the Carnot groups $\mathbb{G}$ of step $\kappa$. A short description of Carnot groups is in Section 2. Here we simply recall that they are connected, simply connected Lie group whose Lie algebra $\mathfrak{g}$ admits a step $\kappa$ stratification. Through the exponential map, a Carnot group $\mathbb{G}$ can be identified with $\mathbb{R}^{N}$, for a certain $N>0$, endowed with a non commutative group operation.

Euclidean spaces are commutative Carnot groups and are the only commutative ones. The simplest but, at the same time, non-trivial instances of non-Abelian Carnot groups are provided by the Heisenberg groups $\mathbb{H}^{n}$ (see for instance [6]).

A Carnot group $\mathbb{G}$ is endowed with a natural left-invariant metric $d$. Non commutative Carnot groups, endowed with their left invariant metric are not Riemannian manifolds not even locally. In fact they are particular instances of so called sub Riemannian manifolds.

Main objects of study in this paper are the notions of regular surfaces and of intrinsic graphs and their link.

Intrinsic regular surfaces in Carnot groups should play the same role as $\mathbb{C}^{1}$ surfaces in Euclidean spaces. In Euclidean spaces, $\mathbb{C}^{1}$ surfaces can be locally defined in different ways: e.g. as non critical level sets of $\mathbb{C}^{1}$ functions or, equivalently, as graphs of $\mathbb{C}^{1}$ maps between complementary linear subspaces. In Carnot groups the equivalence of these definitions is not true any more. One of the main aim of this paper is to find the additional assumptions in order that these notions are equivalent in $\mathbb{G}$. Precisely we want to generalize the results on [2] valid in Heisenberg groups to the more general setting of Carnot groups.

Here by the word intrinsic and regular we want to emphasize a privileged role played by group translations and dilations, and its differential structure as Carnot-Carathéodory manifold in a sense we will precise below.

Date: December 16, 2019.

2010 Mathematics Subject Classification. Primary 35R03; Secondary 28A75, 53C17.

Key words and phrases. Carnot groups, intrinsic differentiability, intrinsic regular surfaces, intrinsic graphs.

D.D.D. is supported by University of Trento, Italy. 
We begin recalling that an intrinsic regular hypersurface (i.e. a topological codimension 1 surface) $S \subset \mathbb{G}$ is locally defined as a non critical level set of a $\mathbb{C}^{1}$ intrinsic function. More precisely, there exists a continuous function $f: \mathbb{G} \rightarrow \mathbb{R}$ such that locally $S=\{P \in \mathbb{G}: f(P)=0\}$ and the intrinsic gradient $\nabla_{\mathbb{G}} f=\left(X_{1} f, \ldots, X_{m} f\right)$ exists in the sense of distributions and it is continuous and non vanishing on $S$. In a similar way, a $k$-codimensional regular surface $S \subset \mathbb{G}$ is locally defined as a non critical level set of a $\mathbb{C}^{1}$ intrinsic vector function $F: \mathbb{G} \rightarrow \mathbb{R}^{k}$.

On the other hand, the intrinsic graphs came out naturally in [12, while studying level sets of Pansu differentiable functions from $\mathbb{H}^{n}$ to $\mathbb{R}$. The simple idea of intrinsic graph is the following one: let $\mathbb{M}$ and $\mathbb{W}$ be complementary subgroups of $\mathbb{G}$, i.e. homogeneous subgroups such that $\mathbb{W} \cap \mathbb{M}=\{0\}$ and $\mathbb{G}=\mathbb{W} \cdot \mathbb{M}$ (here $\cdot$ indicates the group operation in $\mathbb{G}$ and 0 is the unit element), then the intrinsic left graph of $\phi: \mathbb{W} \rightarrow \mathbb{M}$ is the set

$$
\operatorname{graph}(\phi):=\{A \cdot \phi(A) \mid A \in \mathbb{W}\} .
$$

Hence the existence of intrinsic graphs depends on the possibility of splitting $\mathbb{G}$ as a product of complementary subgroups hence it depends on the structure of the algebra $\mathfrak{g}$.

By Implicit Function Theorem, proved in [12] for the Heisenberg group and in [13] for a general Carnot group (see also Theorem 1.3, [22]) it follows

a $\mathbb{G}$-regular surface $S$ locally is an intrinsic graph of a suitable function $\phi$.

Consequently, given an intrinsic graph $S=\operatorname{graph}(\phi) \subset \mathbb{G}$, the main aim of this paper is to find necessary and sufficient assumptions on $\phi$ in order that the opposite implication is true.

Following [27], in Section 3 we define an appropriate notion of differentiability for a map acting between complementary subgroups of $\mathbb{G}$ here denoted as uniform intrinsic differentiability (see Definition [3.3). In Theorem 4.1 we characterize $\mathbb{G}$-regular intrinsic graphs as graphs of uniformly intrinsic differentiable functions $\phi: \mathcal{E} \subset \mathbb{W} \rightarrow \mathbb{M}$ where $\mathbb{G}$ is a step $\kappa$ Carnot group, $\mathbb{W}, \mathbb{M}$ complementary subgroups, with $\mathbb{M}$ horizontal and $k$ dimensional. This result generalizes Theorem 1.2 in 2] proved in Heisenberg groups (see also [28]). As a corollary of this result and Theorem 3.3.5 in [18], we get a comparison between the Reifenberg vanishing flat set and the uniformly intrinsic differentiable map (see Theorem 2.2).

When $\mathbb{M}$ is one dimensional we can identify $\phi: \mathcal{E} \subset \mathbb{W} \rightarrow \mathbb{M}$ with a real valued continuous function defined on an one codimensional homogeneous subgroup of $\mathbb{G}$ (see Remark 5.3). In this case in Heisenberg groups, it is known after the results in [2,5] that the intrinsic differentiability of $\phi$ is equivalent to the existence and continuity of suitable 'derivatives' $D_{j}^{\phi} \phi$ of $\phi$. The non linear first order differential operators $D_{j}^{\phi}$ were introduced by Serra Cassano et al. in the context of Heisenberg groups $\mathbb{H}^{n}$ (see [27] and the references therein). Following the notations in [27] the operators $D_{j}^{\phi}$ are denoted as intrinsic derivatives of $\phi$ and $D^{\phi} \phi$, the vector of the intrinsic derivatives of $\phi$, is the intrinsic gradient of $\phi$. In the first Heisenberg group $\mathbb{H}^{1}$ the intrinsic derivative $D^{\phi} \phi$ reduces to the classical Burgers' equation.

In [2, 4, 5], the authors introduce and investigate some suitable notions of weak solution for the non-linear first order PDEs' system

$$
D^{\phi} \phi=w \quad \text { in } \mathcal{O}
$$

being $w$ a prescribed continuous function and $\mathcal{O} \subset \mathbb{R}^{N-1}$. In [2] and [5] $\phi$ and $w$ are continuous functions, while in [4] $w$ is only a bounded measurable function.

In particular in 22 it was introduced the concept of broad* solution of the system (11) (see Definition 5.3). In $\mathbb{H}^{1}$ this notion extends the classical notion of broad solution for Burgers' equation through characteristic curves provided $\phi$ and $w$ are locally Lipschitz continuous. In our case $\phi$ and $w$ are supposed to be only continuous then the classical theory breaks down. On the other hand broad* $^{*}$ solutions of the system (11) can be constructed with a continuous datum $w$. 
In Section 5 we extend the results in 2 using the notion of broad* solution of the system (1). We study real valued functions defined on an one codimensional homogeneous subgroup of a Carnot group of step 2. We define the appropriate notion of intrinsic derivative in this setting and we extend Theorem 1.3 and Theorem 5.7 in 2 proved in $\mathbb{H}^{n}$. Indeed we analyze the $\mathbb{G}$-regular hypersurfaces in a subclass of step two Carnot groups including the Heisenberg groups. This class is shown in [6] (see Section 5.1). Precisely, in Theorem 5.8 we prove that the intrinsic graph of continuous map $\phi$ is a regular surface if and only if $\phi$ is broad* solution of (1D) and it is $1 / 2$-little Hölder continuous (see Definition 17). We also show that these assumptions are equivalent to the fact that $\phi$ and its intrinsic gradient can be uniformly approximated by $\mathbb{C}^{1}$ functions.

Acknowledgements. We wish to express our gratitude to R.Serapioni and F.Serra Cassano, for having signaled us this problem and for many invaluable discussions during our $\mathrm{PhD}$ at University of Trento. We thank A.Pinamonti for important suggestions on the subject. We also thank B.Franchi e D.Vittone for a careful reading of our $\mathrm{PhD}$ thesis and of this paper.

\section{Notations AND PRELIMINARY RESUlts}

2.1. Carnot groups. We begin by recalling briefly the definition of Carnot groups. For a general account see e.g. [6, 11, 19, 27].

A Carnot group $\mathbb{G}=\left(\mathbb{G}, \cdot, \delta_{\lambda}\right)$ of step $\kappa$ is a connected and simply connected Lie group whose Lie algebra $\mathfrak{g}$ admits a stratification, i.e. a direct sum decomposition $\mathfrak{g}=V_{1} \oplus V_{2} \oplus \cdots \oplus V_{\kappa}$. The stratification has the further property that the entire Lie algebra $\mathfrak{g}$ is generated by its first layer $V_{1}$, the so called horizontal layer, that is

$$
\left\{\begin{array}{l}
{\left[V_{1}, V_{i-1}\right]=V_{i} \quad \text { if } 2 \leq i \leq \kappa} \\
{\left[V_{1}, V_{\kappa}\right]=\{0\}}
\end{array}\right.
$$

We denote by $N$ the dimension of $\mathfrak{g}$ and by $n_{s}$ the dimension of $V_{s}$.

The exponential map exp $: \mathfrak{g} \rightarrow \mathbb{G}$ is a global diffeomorphism from $\mathfrak{g}$ to $\mathbb{G}$. Hence, if we choose a basis $\left\{X_{1}, \ldots, X_{N}\right\}$ of $\mathfrak{g}$, any $P \in \mathbb{G}$ can be written in a unique way as $P=\exp \left(p_{1} X_{1}+\cdots+p_{N} X_{N}\right)$ and we can identify $P$ with the $N$-tuple $\left(p_{1}, \ldots, p_{N}\right) \in \mathbb{R}^{N}$ and $\mathbb{G}$ with $\left(\mathbb{R}^{N}, \cdot, \delta_{\lambda}\right)$. The identity of $\mathbb{G}$ is the origin of $\mathbb{R}^{N}$.

For any $\lambda>0$, the (non isotropic) dilation $\delta_{\lambda}: \mathbb{G} \rightarrow \mathbb{G}$ are automorhisms of $\mathbb{G}$ and are defined as

$$
\delta_{\lambda}\left(p_{1}, \ldots, p_{N}\right)=\left(\lambda^{\alpha_{1}} p_{1}, \ldots, \lambda^{\alpha_{N}} p_{N}\right)
$$

where $\alpha_{i} \in \mathbb{N}$ is called homogeneity of the variable $p_{i}$ in $\mathbb{G}$ and is given by $\alpha_{i}=j$ whenever $m_{j-1}<$ $i \leq m_{j}$ with $m_{s}-m_{s-1}=n_{s}$ and $m_{0}=0$. Hence $1=\alpha_{1}=\cdots=\alpha_{m_{1}}<\alpha_{m_{1}+1}=2 \leq \cdots \leq \alpha_{N}=\kappa$.

The explicit expression of the group operation · is determined by the Campbell-Hausdorff formula. It has the form

$$
P \cdot Q=P+Q+\mathcal{Q}(P, Q) \text { for all } P, Q \in \mathbb{G} \equiv \mathbb{R}^{N},
$$

where $\mathcal{Q}=\left(\mathcal{Q}_{1}, \ldots, \mathcal{Q}_{N}\right): \mathbb{R}^{N} \times \mathbb{R}^{N} \rightarrow \mathbb{R}^{N}$ and every $\mathcal{Q}_{i}$ is a homogeneous polynomial of degree $\alpha_{i}$ with respect to the intrinsic dilations of $\mathbb{G}$, i.e.

$$
\mathcal{Q}_{i}\left(\delta_{\lambda} P, \delta_{\lambda} Q\right)=\lambda^{\alpha_{i}} \mathcal{Q}_{i}(P, Q) \text { for all } P, Q \in \mathbb{G} \text { and } \lambda>0 .
$$

We collect now further properties of $\mathcal{Q}$ following from Campbell-Hausdorff formula. First of all $\mathcal{Q}$ is antisymmetric, that is

$$
\mathcal{Q}_{i}(P, Q)=-\mathcal{Q}_{i}(-P,-Q) \text { for all } P, Q \in \mathbb{G} .
$$

Moreover

$$
\mathcal{Q}_{i}(P, 0)=\mathcal{Q}_{i}(0, P)=0 \quad \text { and } \mathcal{Q}_{i}(P, P)=\mathcal{Q}_{i}(P,-P)=0
$$


and each $\mathcal{Q}_{i}(P, Q)$ depend only on the first components of $P$ and $Q$. More precisely if $m_{i-1}<s \leq m_{i}$ and $i \geq 2$,

$$
\begin{aligned}
& \mathcal{Q}_{1}(P, Q)=\cdots=\mathcal{Q}_{m_{1}}(P, Q)=0 \\
& \mathcal{Q}_{s}(P, Q)=\mathcal{Q}_{s}\left(\left(p_{1}, \ldots, p_{m_{i-1}}\right),\left(q_{1}, \ldots, q_{m_{i-1}}\right)\right) .
\end{aligned}
$$

Observe also that $\mathbb{G}=\mathbb{G}^{1} \oplus \mathbb{G}^{2} \oplus \cdots \oplus \mathbb{G}^{\kappa}$ where $\mathbb{G}^{i}=\exp \left(V_{i}\right)=\mathbb{R}^{n_{i}}$ is the $i^{\text {th }}$ layer of $\mathbb{G}$ and to write $P \in \mathbb{G}$ as $\left(P^{1}, \ldots, P^{\kappa}\right)$ with $P^{i} \in \mathbb{G}^{i}$. According to this

(3) $P \cdot Q=\left(P^{1}+Q^{1}, P^{2}+Q^{2}+\mathcal{Q}^{2}\left(P^{1}, Q^{1}\right), \ldots, P^{\kappa}+Q^{\kappa}+\mathcal{Q}^{\kappa}\left(\left(P^{1}, \ldots, P^{\kappa-1}\right),\left(Q^{1}, \ldots, Q^{\kappa-1}\right)\right)\right.$ for every $P=\left(P^{1}, \ldots, P^{\kappa}\right), Q=\left(Q^{1}, \ldots, Q^{\kappa}\right) \in \mathbb{G}$. In particular $P^{-1}=\left(-P^{1}, \ldots,-P^{\kappa}\right)$.

The norm of $\mathbb{R}^{n_{s}}$ is denoted with the symbol $|\cdot| \mathbb{R}^{n_{s}}$.

For any $P \in \mathbb{G}$ the intrinsic left translation $\tau_{P}: \mathbb{G} \rightarrow \mathbb{G}$ are defined as

$$
Q \mapsto \tau_{P} Q:=P \cdot Q=P Q .
$$

A homogeneous norm on $\mathbb{G}$ is a nonnegative function $P \mapsto\|P\|$ such that for all $P, Q \in \mathbb{G}$ and for all $\lambda \geq 0$

$$
\begin{gathered}
\|P\|=0 \quad \text { if and only if } P=0 \\
\left\|\delta_{\lambda} P\right\|=\lambda\|P\|, \quad\|P \cdot Q\| \leq\|P\|+\|Q\| .
\end{gathered}
$$

There is a particular homogeneous norm defined in Theorem 5.1 in [15], as following

$$
\left\|\left(P^{1}, \ldots, P^{\kappa}\right)\right\|:=\max _{s=1, \ldots, \kappa}\left\{\epsilon_{s}\left|P^{s}\right|_{\mathbb{R}^{n_{s}}}^{1 / s}\right\} \quad \text { for all }\left(P^{1}, \ldots, P^{\kappa}\right) \in \mathbb{G}
$$

with $\epsilon_{1}=1$, and $\epsilon_{s} \in(0,1]$ depending on the structure of the group for $s=2, \ldots, \kappa$. This homogeneous norm is symmetric, i.e. $\|P\|=\left\|P^{-1}\right\|$ for all $P \in \mathbb{G}$ and such that

$$
\left\|\left(P^{1}, 0 \ldots, 0\right)\right\|=\left|P^{1}\right|_{\mathbb{R}^{m_{1}}},
$$

for all $P^{1} \in \mathbb{R}^{m_{1}}$.

W.l.o.g. we consider the homogeneous norm defined in (44). Indeed from the fact that all the homogeneous norms are equivalent, all the estimates we will use hold with an arbitrary homogeneous norm.

Given any homogeneous norm $\|\cdot\|$, it is possible to introduce a distance in $\mathbb{G}$ given by

$$
d(P, Q)=d\left(P^{-1} Q, 0\right)=\left\|P^{-1} Q\right\| \quad \text { for all } P, Q \in \mathbb{G} .
$$

We observe that any distance $d$ obtained in this way is always equivalent with the Carnot-Carathéodory's distance $d_{c c}$ of the group (see Proposition 5.1.4 and Theorem 5.2.8 [6]).

The distance $d$ is well behaved with respect to left translations and dilations, i.e. for all $P, Q, Q^{\prime} \in$ $\mathbb{G}$ and $\lambda>0$,

$$
d\left(P \cdot Q, P \cdot Q^{\prime}\right)=d\left(Q, Q^{\prime}\right), \quad d\left(\delta_{\lambda} Q, \delta_{\lambda} Q^{\prime}\right)=\lambda d\left(Q, Q^{\prime}\right)
$$

Moreover, by Proposition 5.15.1 in [6], for any bounded subset $\Omega \subset \mathbb{G}$ there exist positive constants $c_{1}=c_{1}(\Omega), c_{2}=c_{2}(\Omega)$ such that for all $P, Q \in \Omega$

$$
c_{1}|P-Q|_{\mathbb{R}^{N}} \leq d(P, Q) \leq c_{2}|P-Q|_{\mathbb{R}^{N}}^{1 / \kappa}
$$

and, in particular, the topology induced on $\mathbb{G}$ by $d$ is the Euclidean topology.

We also define the distance dist $_{d}$ between two set $\Omega_{1}, \Omega_{2} \subset \mathbb{G}$ by putting

$$
\operatorname{dist}_{d}\left(\Omega_{1}, \Omega_{2}\right):=\max \left\{\sup _{Q^{\prime} \in \Omega_{2}} d\left(\Omega_{1}, Q^{\prime}\right), \sup _{Q \in \Omega_{1}} d\left(Q, \Omega_{2}\right)\right\},
$$

where $d\left(\Omega_{1}, Q^{\prime}\right):=\inf \left\{d\left(Q, Q^{\prime}\right): Q \in \Omega_{1}\right\}$.

The Hausdorff dimension of $(\mathbb{G}, d)$ as a metric space is denoted homogeneous dimension of $\mathbb{G}$ and it can be proved to be the integer $\sum_{j=1}^{N} \alpha_{j}=\sum_{i=1}^{\kappa} i \operatorname{dim} V_{i} \geq N$ (see [24]). 
The subbundle of the tangent bundle $T \mathbb{G}$, spanned by the vector fields $X_{1}, \ldots, X_{m_{1}}$ plays a particularly important role in the theory, and is called the horizontal bundle $H \mathbb{G}$; the fibers of $H \mathbb{G}$ are

$$
H \mathbb{G}_{P}=\operatorname{span}\left\{X_{1}(P), \ldots, X_{m_{1}}(P)\right\}, \quad P \in \mathbb{G} .
$$

A sub Riemannian structure is defined on $\mathbb{G}$, endowing each fiber of $H \mathbb{G}$ with a scalar product $\langle\cdot, \cdot\rangle_{P}$ and a norm $|\cdot|_{P}$ making the basis $X_{1}(P), \ldots, X_{m_{1}}(P)$ an orthonormal basis. Hence, if $v=\sum_{i=1}^{m_{1}} v_{i} X_{i}(P)=v^{1}$ and $w=\sum_{i=1}^{m_{1}} w_{i} X_{i}(P)=w^{1}$ are in $H \mathbb{G}$, then $\langle v, w\rangle_{P}:=\sum_{i=1}^{m_{1}} v_{i} w_{i}$ and $|v|_{P}^{2}:=\langle v, v\rangle_{P}$. We will write, with abuse of notation, $\langle\cdot, \cdot\rangle$ meaning $\langle\cdot, \cdot\rangle_{P}$ and $|\cdot|$ meaning $|\cdot|_{P}$.

The sections of $H \mathbb{G}$ are called horizontal sections, a vector of $H \mathbb{G}_{P}$ is an horizontal vector while any vector in $T \mathbb{G}_{P}$ that is not horizontal is a vertical vector.

The Haar measure of the group $\mathbb{G}=\mathbb{R}^{N}$ is the Lebesgue measure $d \mathcal{L}^{N}$. It is left (and right) invariant. Various Lebesgue spaces on $\mathbb{G}$ are meant always with respect to the measure $d \mathcal{L}^{N}$ and are denoted as $\mathcal{L}^{p}(\mathbb{G})$.

2.2. $\mathbb{C}_{\mathbb{G}}^{1}$ functions, $\mathbb{G}$-regular surfaces, Caccioppoli sets. (See [21, 27]). In [25] Pansu introduced an appropriate notion of differentiability for functions acting between Carnot groups. We recall this definition in the particular instance that is relevant here.

Let $\mathcal{U}$ be an open subset of a Carnot group $\mathbb{G}$. A function $f: \mathcal{U} \rightarrow \mathbb{R}^{k}$ is Pansu differentiable or more simply $\mathrm{P}$-differentiable in $A_{0} \in \mathcal{U}$ if there is a homogeneous homomorphism

$$
d_{\mathbf{P}} f\left(A_{0}\right): \mathbb{G} \rightarrow \mathbb{R}^{k}
$$

the Pansu differential of $f$ in $A_{0}$, such that, for $B \in \mathcal{U}$,

$$
\lim _{r \rightarrow 0^{+}} \sup _{0<\left\|A_{0}^{-1} B\right\|<r} \frac{\left|f(B)-f\left(A_{0}\right)-d_{\mathbf{P}} f\left(A_{0}\right)\left(A_{0}^{-1} B\right)\right|_{\mathbb{R}^{k}}}{\left\|A_{0}^{-1} B\right\|}=0 .
$$

Saying that $d_{\mathbf{P}} f\left(A_{0}\right)$ is a homogeneous homomorphism we mean that $d_{\mathbf{P}} f\left(A_{0}\right): \mathbb{G} \rightarrow \mathbb{R}^{k}$ is a group homomorphism and also that $d_{\mathbf{P}} f\left(A_{0}\right)\left(\delta_{\lambda} B\right)=\lambda d_{\mathbf{P}} f\left(A_{0}\right)(B)$ for all $B \in \mathbb{G}$ and $\lambda \geq 0$.

Observe that, later on in Definition 3.2, we give a different notion of differentiability for functions acting between subgroups of a Carnot group and we reserve the notation $d f$ or $d f\left(A_{0}\right)$ for that differential.

We denote $\mathbb{C}_{\mathbb{G}}^{1}\left(\mathcal{U}, \mathbb{R}^{k}\right)$ the set of functions $f: \mathcal{U} \rightarrow \mathbb{R}^{k}$ that are P-differentiable in each $A \in \mathcal{U}$ and such that $d_{\mathbf{P}} f(A)$ depends continuously on $A$.

It can be proved that $f=\left(f_{1}, \ldots, f_{k}\right) \in \mathbb{C}_{\mathbb{G}}^{1}\left(\mathcal{U}, \mathbb{R}^{k}\right)$ if and only if the distributional horizontal derivatives $X_{i} f_{j}$, for $i=1 \ldots, m_{1}, j=1, \ldots, k$, are continuous in $\mathcal{U}$. Remember that $\mathbb{C}^{1}(\mathcal{U}, \mathbb{R}) \subset$ $\mathbb{C}_{\mathbb{G}}^{1}(\mathcal{U}, \mathbb{R})$ with strict inclusion whenever $\mathbb{G}$ is not abelian (see Remark 6 in [12]).

The horizontal Jacobian (or the horizontal gradient if $k=1$ ) of $f: \mathcal{U} \rightarrow \mathbb{R}^{k}$ in $A \in \mathcal{U}$ is the matrix

$$
\nabla_{\mathbb{G}} f(A):=\left[X_{i} f_{j}(A)\right]_{i=1 \ldots m_{1}, j=1 \ldots k}
$$

when the partial derivatives $X_{i} f_{j}$ exist. Hence $f=\left(f_{1}, \ldots, f_{k}\right) \in \mathbb{C}_{\mathbb{G}}^{1}\left(\mathcal{U}, \mathbb{R}^{k}\right)$ if and only if its horizontal Jacobian exists and is continuous in $\mathcal{U}$. The horizontal divergence of $\phi:=\left(\phi_{1}, \ldots, \phi_{m_{1}}\right)$ : $\mathcal{U} \rightarrow \mathbb{R}^{m_{1}}$ is defined as

$$
\operatorname{div}_{\mathbb{G}} \phi:=\sum_{j=1}^{m_{1}} X_{j} \phi_{j}
$$

if $X_{j} \phi_{j}$ exist for $j=1, \ldots, m_{1}$.

The following proposition shows that the P-differential of a P-differentiable map $f$ is represented by horizontal gradient $\nabla_{\mathbb{G}} f$ : 
Proposition 2.1. If $f: \mathcal{U} \subset \mathbb{G} \rightarrow \mathbb{R}$ is P-differentiable at a point $P$ and $d_{\mathbf{P}} f(P)$ is $P$-differential of $f$ at $P$, then

$$
d_{\mathbf{P}} f(P)(Q)=\nabla_{\mathbb{G}} f(P) Q^{1}, \quad \text { for all } Q=\left(Q^{1}, \ldots, Q^{\kappa}\right) \in \mathcal{U} .
$$

In the setting of Carnot groups, there is a natural definition of bounded variation functions and of finite perimeter sets (see [16] or [27] and the bibliography therein).

We say that $f: \mathcal{U} \rightarrow \mathbb{R}$ is of bounded $\mathbb{G}$-variation in an open set $\mathcal{U} \subset \mathbb{G}$ and we write $f \in B V_{\mathbb{G}}(\mathcal{U})$, if $f \in \mathcal{L}^{1}(\mathcal{U})$ and

$$
\left\|\nabla_{\mathbb{G}} f\right\|(\mathcal{U}):=\sup \left\{\int_{\mathcal{U}} f \operatorname{div}_{\mathbb{G}} \phi d \mathcal{L}^{N}: \phi \in \mathbb{C}_{c}^{1}(\mathcal{U}, H \mathbb{G}),|\phi(P)| \leq 1 \text { for all } P \in \mathcal{U}\right\}<+\infty .
$$

The space $B V_{\mathbb{G}, l o c}(\mathcal{U})$ is defined in the usual way. A set $\mathcal{E} \subset \mathbb{G}$ has locally finite $\mathbb{G}$-perimeter, or is a $\mathbb{G}$-Caccioppoli set, if $\chi_{\mathcal{E}} \in B V_{\mathbb{G}, l o c}(\mathbb{G})$, where $\chi_{\mathcal{E}}$ is the characteristic function of the set $\mathcal{E}$. In this case the measure $\left\|\nabla_{\mathbb{G}} \chi_{\mathcal{E}}\right\|$ is called the $\mathbb{G}$-perimeter measure of $\mathcal{E}$ and is denoted by $|\partial \mathcal{E}|_{\mathbb{G}}$.

Now we define co-abelian intrinsic submanifold as in Definition 3.3.4 [18]. Following the terminology of [14], we call these objects $k$-codimensional $\mathbb{G}$-regular surfaces.

Definition 2.1. $S \subset \mathbb{G}$ is a $k$-codimensional $\mathbb{G}$-regular surface if for every $P \in S$ there are a neighbourhood $\mathcal{U}$ of $P$ and a function $f=\left(f_{1}, \ldots, f_{k}\right) \in \mathbb{C}_{\mathbb{G}}^{1}\left(\mathcal{U}, \mathbb{R}^{k}\right)$ such that

$$
S \cap \mathcal{U}=\{Q \in \mathcal{U}: f(Q)=0\}
$$

and $d_{\mathbf{P}} f(Q)$ is surjective, or equivalently if the $\left(k \times m_{1}\right)$ matrix $\nabla_{\mathbb{G}} f(Q)$ has rank $k$, with $k<m_{1}$, for all $Q \in \mathcal{U}$.

The class of $\mathbb{G}$-regular surfaces is different from the class of Euclidean regular surfaces. In [17], the authors give an example of $\mathbb{H}^{1}$-regular surfaces, in $\mathbb{H}^{1}$ identified with $\mathbb{R}^{3}$, that are (Euclidean) fractal sets. Conversely, there are continuously differentiable 2 -submanifolds in $\mathbb{R}^{3}$ that are not $\mathbb{H}^{1}$-regular surfaces (see [12] Remark 6.2 and [2] Corollary 5.11).

Recall that a homogeneous subgroup $\mathbb{W}$ of $\mathbb{G}$ is a Lie subgroup such that $\delta_{\lambda} A \in \mathbb{W}$ for every $A \in \mathbb{W}$ and for all $\lambda>0$, we can give the following result about $\mathbb{G}$-regular surface:

Theorem 2.2 (see [18], Theorem 3.3.5). Let $S \subset \mathbb{G}$ be a closed connected set. The following conditions are equivalent:

(1) $S$ is a $k$-codimensional $\mathbb{G}$-regular surface

(2) $S$ is Reifenberg vanishing flat with respect to a family of closed homogeneous subsets $\left\{\mathbb{W}_{P}\right.$ : $P \in S\}$ such that $\mathbb{W}_{P}$ is a vertical subgroup of codimension $k$ for some $P \in S$, i.e. for every relatively compact subset $S^{\prime} \Subset S$ there is an increasing function $\beta:(0, \infty) \rightarrow(0, \infty)$, with $\beta(t) \rightarrow 0^{+}$when $t \rightarrow 0^{+}$, such that

$$
\operatorname{dist}_{d}\left(\mathcal{U}(P, r) \cap S, \mathcal{U}(P, r) \cap\left(P \cdot \mathbb{W}_{P}\right)\right) \leq \beta(r) r, \quad r>0
$$

for any $P \in S^{\prime}$. then

In particular, if (1) or equivalently (2) hold, i.e. $S$ is locally level set of a certain $f \in \mathbb{C}_{\mathbb{G}}^{1}\left(\mathbb{G}, \mathbb{R}^{k}\right)$

$$
\mathbb{W}_{P}=\operatorname{ker}\left(d_{\mathbf{P}} f(P)\right), \quad \forall P \in S .
$$

\subsection{Complementary subgroups and graphs.}

Definition 2.2. We say that $\mathbb{W}$ and $\mathbb{M}$ are complementary subgroups in $\mathbb{G}$ if $\mathbb{W}$ and $\mathbb{M}$ are homogeneous subgroups of $\mathbb{G}$ such that $\mathbb{W} \cap \mathbb{M}=\{0\}$ and

$$
\mathbb{G}=\mathbb{W} \cdot \mathbb{M} \text {. }
$$

By this we mean that for every $P \in \mathbb{G}$ there are $P_{\mathbb{W}} \in \mathbb{W}$ and $P_{\mathbb{M}} \in \mathbb{M}$ such that $P=P_{\mathbb{W}} P_{\mathbb{M}}$. 
The elements $P_{\mathbb{W}} \in \mathbb{W}$ and $P_{\mathbb{M}} \in \mathbb{M}$ such that $P=P_{\mathbb{W}} \cdot P_{\mathbb{M}}$ are unique because of $\mathbb{W} \cap \mathbb{M}=\{0\}$ and are denoted components of $P$ along $\mathbb{W}$ and $\mathbb{M}$ or projections of $P$ on $\mathbb{W}$ and $\mathbb{M}$. The projection maps $\mathbf{P}_{\mathbb{W}}: \mathbb{G} \rightarrow \mathbb{W}$ and $\mathbf{P}_{\mathbb{M}}: \mathbb{G} \rightarrow \mathbb{M}$ defined

$$
\mathbf{P}_{\mathbb{W}}(P)=P_{\mathbb{W}}, \quad \mathbf{P}_{\mathbb{M}}(P)=P_{\mathbb{M}}, \quad \text { for all } P \in \mathbb{G}
$$

are polynomial functions (see Proposition 2.2.14 in [11]) if we identify $\mathbb{G}$ with $\mathbb{R}^{N}$, hence are $\mathbb{C}^{\infty}$. Nevertheless in general they are not Lipschitz maps, when $\mathbb{W}$ and $\mathbb{M}$ are endowed with the restriction of the left invariant distance $d$ of $\mathbb{G}$ (see Example 2.2.15 in [11]).

Remark 2.3. The stratification of $\mathbb{G}$ induces a stratifications on the complementary subgroups $\mathbb{W}$ and $\mathbb{M}$. If $\mathbb{G}=\mathbb{G}^{1} \oplus \cdots \oplus \mathbb{G}^{\kappa}$ then also $\mathbb{W}=\mathbb{W}^{1} \oplus \cdots \oplus \mathbb{W}^{\kappa}, \mathbb{M}=\mathbb{M}^{1} \oplus \cdots \oplus \mathbb{M}^{\kappa}$ and $\mathbb{G}^{i}=\mathbb{W}^{i} \oplus \mathbb{M}^{i}$. A subgroup is horizontal if it is contained in the first layer $\mathbb{G}^{1}$. If $\mathbb{M}$ is horizontal then the complementary subgroup $\mathbb{W}$ is normal.

Proposition 2.4 (see [3], Proposition 3.2). If $\mathbb{W}$ and $\mathbb{M}$ are complementary subgroups in $\mathbb{G}$ there is $c_{0}=c_{0}(\mathbb{W}, \mathbb{M}) \in(0,1)$ such that for each $P_{\mathbb{W}} \in \mathbb{W}$ and $P_{\mathbb{M}} \in \mathbb{M}$

$$
c_{0}\left(\left\|P_{\mathbb{W}}\right\|+\left\|P_{\mathbb{M}}\right\|\right) \leq\left\|P_{\mathbb{W}} P_{\mathbb{M}}\right\| \leq\left\|P_{\mathbb{W}}\right\|+\left\|P_{\mathbb{M}}\right\|
$$

Definition 2.3. We say that $S \subset \mathbb{G}$ is a left intrinsic graph or more simply a intrinsic graph if there are complementary subgroups $\mathbb{W}$ and $\mathbb{M}$ in $\mathbb{G}$ and $\phi: \mathcal{E} \subset \mathbb{W} \rightarrow \mathbb{M}$ such that

$$
S=\operatorname{graph}(\phi):=\{A \phi(A): A \in \mathcal{E}\} .
$$

Observe that, by uniqueness of the components along $\mathbb{W}$ and $\mathbb{M}$, if $S=\operatorname{graph}(\phi)$ then $\phi$ is uniquely determined among all functions from $\mathbb{W}$ to $\mathbb{M}$.

Proposition 2.5 (see Proposition 2.2.18 in [11]). If $S$ is a intrinsic graph then, for all $\lambda>0$ and for all $Q \in \mathbb{G}, Q \cdot S$ and $\delta_{\lambda} S$ are intrinsic graphs. In particular, if $S=\operatorname{graph}(\phi)$ with $\phi: \mathcal{E} \subset \mathbb{W} \rightarrow \mathbb{M}$, then

(1) For all $\lambda>0$,

$$
\delta_{\lambda}(\operatorname{graph}(\phi))=\operatorname{graph}\left(\phi_{\lambda}\right)
$$

where $\phi_{\lambda}: \delta_{\lambda} \mathcal{E} \subset \mathbb{W} \rightarrow \mathbb{M}$ and $\phi_{\lambda}(A):=\delta_{\lambda} \phi\left(\delta_{1 / \lambda} A\right)$, for $A \in \delta_{\lambda} \mathcal{E}$.

(2) For any $Q \in \mathbb{G}$,

$$
Q \cdot \operatorname{graph}(\phi)=\operatorname{graph}\left(\phi_{Q}\right)
$$

where $\phi_{Q}: \mathcal{E}_{Q} \subset \mathbb{W} \rightarrow \mathbb{M}$ is defined as $\phi_{Q}(A):=\left(\mathbf{P}_{\mathbb{M}}\left(Q^{-1} A\right)\right)^{-1} \phi\left(\mathbf{P}_{\mathbb{W}}\left(Q^{-1} A\right)\right)$, for all $A \in \mathcal{E}_{Q}:=\left\{A: \mathbf{P}_{\mathbb{W}}\left(Q^{-1} A\right) \in \mathcal{E}\right\}$.

The following notion of intrinsic Lipschitz function appeared for the first time in [12] and was studied, more diffusely, in [4, 5, 10, 11, 14, 26]. Intrinsic Lipschitz functions play the same role as Lipschitz functions in Euclidean context.

Definition 2.4. Let $\mathbb{W}, \mathbb{M}$ be complementary subgroups in $\mathbb{G}, \phi: \mathcal{E} \subset \mathbb{W} \rightarrow \mathbb{M}$. We say that $\phi$ is intrinsic $C_{L}$-Lipschitz in $\mathcal{E}$, or simply intrinsic Lipschitz, if there is $C_{L} \geq 0$ such that

$$
\left\|\mathbf{P}_{\mathbb{M}}\left(Q^{-1} Q^{\prime}\right)\right\| \leq C_{L}\left\|\mathbf{P}_{\mathbb{W}}\left(Q^{-1} Q^{\prime}\right)\right\|, \quad \text { for all } Q, Q^{\prime} \in \operatorname{graph}(\phi) .
$$

$\phi: \mathcal{E} \rightarrow \mathbb{M}$ is locally intrinsic Lipschitz in $\mathcal{E}$ if $\phi$ is intrinsic Lipschitz in $\mathcal{E}^{\prime}$ for every $\mathcal{E}^{\prime} \Subset \mathcal{E}$.

Remark 2.6. In this paper we are interested mainly in the special case when $\mathbb{M}$ is a horizontal subgroup and consequently $\mathbb{W}$ is a normal subgroup. Under these assumptions, for all $P=A \phi(A), Q=$ $B \phi(B) \in \operatorname{graph}(\phi)$ we have

$$
\mathbf{P}_{\mathbb{M}}\left(P^{-1} Q\right)=\phi(A)^{-1} \phi(B), \quad \mathbf{P}_{\mathbb{W}}\left(P^{-1} Q\right)=\phi(A)^{-1} A^{-1} B \phi(A) .
$$

Hence, if $\mathbb{M}$ is a horizontal subgroup, $\phi: \mathcal{E} \subset \mathbb{W} \rightarrow \mathbb{M}$ is intrinsic Lipschitz if

$$
\left\|\phi(A)^{-1} \phi(B)\right\| \leq C_{L}\left\|\phi(A)^{-1} A^{-1} B \phi(A)\right\| \quad \text { for all } A, B \in \mathcal{E} .
$$


Moreover, if $\phi$ is intrinsic Lipschitz then $\left\|\phi(A)^{-1} A^{-1} B \phi(A)\right\|$ is comparable with $\left\|P^{-1} Q\right\|$. Indeed from (5)

$$
\begin{aligned}
c_{0}\left\|\phi(A)^{-1} A^{-1} B \phi(A)\right\| & \leq\left\|P^{-1} Q\right\| \\
& \leq\left\|\phi(A)^{-1} A^{-1} B \phi(A)\right\|+\left\|\phi(A)^{-1} \phi(B)\right\| \\
& \leq\left(1+C_{L}\right)\left\|\phi(A)^{-1} A^{-1} B \phi(A)\right\| .
\end{aligned}
$$

The quantity $\left\|\phi(A)^{-1} A^{-1} B \phi(A)\right\|$, or better a symmetrized version of it, can play the role of a $\phi$ dependent, quasi distance on $\mathcal{E}$. See e.g. [2].

In Euclidean spaces, i.e. when $\mathbb{G}$ is $\mathbb{R}^{N}$ and the group operation is the usual Euclidean sum of vectors, intrinsic Lipschitz functions are the same as Lipschitz functions. On the contrary, when $\mathbb{G}$ is a general non commutative Carnot group and $\mathbb{W}$ and $\mathbb{M}$ are complementary subgroups, the class of intrinsic Lipschitz functions from $\mathbb{W}$ to $\mathbb{M}$ is different from the class Lipschitz functions (see Example 2.3.9 in [10]). More precisely, if $\phi: \mathbb{W} \rightarrow \mathbb{M}$ is intrinsic Lipschitz then in general does not exists a constant $C$ such that

$$
\left\|\phi(A)^{-1} \phi(B)\right\| \leq C\left\|A^{-1} B\right\| \quad \text { for } A, B \in \mathbb{W}
$$

not even locally. Nevertheless the following weaker result holds true:

Proposition 2.7 (see Proposition 3.1.8 in [1]). Let $\mathbb{W}, \mathbb{M}$ be complementary subgroups in a step $\kappa$ Carnot group $\mathbb{G}$. Let $\phi: \mathcal{E} \subset \mathbb{W} \rightarrow \mathbb{M}$ be an intrinsic $C_{L}$-Lipschitz function. Then, for all $r>0$,

(1) there is $C_{1}=C_{1}(\phi, r)>0$ such that

$$
\|\phi(A)\| \leq C_{1} \quad \text { for all } A \in \mathcal{E} \text { with }\|A\| \leq r
$$

(2) there is $C_{2}=C_{2}\left(C_{L}, r\right)>0$ such that $\phi$ is locally $1 / \kappa$-Hölder continuous i.e.

$$
\left\|\phi(A)^{-1} \phi(B)\right\| \leq C_{2}\left\|A^{-1} B\right\|^{1 / \kappa} \quad \text { for all } A, B \text { with }\|A\|,\|B\| \leq r .
$$

\section{INTRINSIC DIFFERENTIABILITY}

The notion of $P$-differentiability makes sense and can be introduced also for functions acting between complementary subgroups of a Carnot group $\mathbb{G}$. Nevertheless $P$-differentiability does not seem to be the right notion in this context. Indeed P-differentiability is a property that can be lost after a function is shifted as in Proposition 2.5. Here we recall a different notion of differentiability, the so called intrinsic differentiability that is, by its very definition, invariant under translations. A function is intrinsic differentiable if it is locally well approximated by intrinsic linear functions that are functions whose graph is a homogeneous subgroup in $\mathbb{G}$.

Definition 3.1. Let $\mathbb{W}$ and $\mathbb{M}$ be complementary subgroups in $\mathbb{G}$. Then $\ell: \mathbb{W} \rightarrow \mathbb{M}$ is intrinsic linear if $\ell$ is defined on all of $\mathbb{W}$ and if graph $(\ell)$ is a homogeneous subgroup of $\mathbb{G}$.

Intrinsic linear functions can be algebraically caracterized as follows.

Proposition 3.1 (see Propositions 3.1.3 and 3.1.6 in [10]). Let $\mathbb{W}$ and $\mathbb{M}$ be complementary subgroups in $\mathbb{G}$. Then $\ell: \mathbb{W} \rightarrow \mathbb{M}$ is intrinsic linear if and only if

$$
\begin{aligned}
& \ell\left(\delta_{\lambda} A\right)=\delta_{\lambda}(\ell(A)), \quad \text { for all } A \in \mathbb{W} \text { and } \lambda \geq 0 \\
& \ell(A B)=\left(\mathbf{P}_{\mathbb{H}}\left(\ell(A)^{-1} B\right)\right)^{-1} \ell\left(\mathbf{P}_{\mathbb{W}}\left(\ell(A)^{-1} B\right)\right), \quad \text { for all } A, B \in \mathbb{W} .
\end{aligned}
$$

Moreover any intrinsic linear function $\ell$ is a polynomial function and it is intrinsic Lipschitz with Lipschitz constant $C_{L}:=\sup \{\|\ell(A)\|:\|A\|=1\}$. Note that $C_{L}<+\infty$ because $\ell$ is continuous. Moreover

$$
\|\ell(A)\| \leq C_{L}\|A\|, \quad \text { for all } A \in \mathbb{W} .
$$


In particular, if $\mathbb{W}$ is normal in $\mathbb{G}$ then $\ell: \mathbb{W} \rightarrow \mathbb{M}$ is intrinsic linear if and only if

$$
\begin{aligned}
& \ell\left(\delta_{\lambda} A\right)=\delta_{\lambda}(\ell(A)), \quad \text { for all } A \in \mathbb{W} \text { and } \lambda \geq 0 \\
& \ell(A B)=\ell(A) \ell\left(\ell(A)^{-1} B \ell(A)\right), \quad \text { for all } A, B \in \mathbb{W} .
\end{aligned}
$$

We use intrinsic linear functions to define intrinsic differentiability as in the usual definition of differentiability.

Definition 3.2. Let $\mathbb{W}$ and $\mathbb{M}$ be complementary subgroups in $\mathbb{G}$ and let $\phi: \mathcal{O} \subset \mathbb{W} \rightarrow \mathbb{M}$ with $\mathcal{O}$ open in $\mathbb{W}$. For $A \in \mathcal{O}$, let $P:=A \cdot \phi(A)$ and $\phi_{P^{-1}}: \mathcal{O}_{P^{-1}} \subset \mathbb{W} \rightarrow \mathbb{M}$ be the shifted function defined in Proposition 2.5. We say that $\phi$ is intrinsic differentiable in $A$ if the shifted function $\phi_{P^{-1}}$ is intrinsic differentiable in 0, i.e. if there is a intrinsic linear $d \phi_{A}: \mathbb{W} \rightarrow \mathbb{M}$ such that

$$
\lim _{r \rightarrow 0^{+}} \sup _{0<\|B\|<r} \frac{\left\|d \phi_{A}(B)^{-1} \phi_{P^{-1}}(B)\right\|}{\|B\|}=0 .
$$

The function $d \phi_{A}$ is the intrinsic differential of $\phi$ at $A$.

Remark 3.2. Definition 3.2 is a natural one because of the following observations.

(i) If $\phi$ is intrinsic differentiable in $A \in \mathcal{O}$, there is a unique intrinsic linear function $d \phi_{A}$ satisfying (77). Moreover $\phi$ is continuous at $A$. (See Theorem 3.2.8 and Proposition 3.2.3 in [10]).

(ii) The notion of intrinsic differentiability is invariant under group translations. Precisely, let $P:=A \phi(A), Q:=B \phi(B)$, then $\phi$ is intrinsic differentiable in $A$ if and only if $\phi_{Q P^{-1}}:=\left(\phi_{P^{-1}}\right)_{Q}$ is intrinsic differentiable in $B$.

(iii) The analytic definition of intrinsic differentiability of Definition 3.2 has an equivalent geometric formulation. Indeed intrinsic differentiability in one point is equivalent to the existence of a tangent subgroup to the graph (see [10], Theorem 3.2.8). Let $\phi: \mathbb{W} \rightarrow \mathbb{M}$ be such that $\phi(0)=0$. We say that an homogeneous subgroup $\mathbb{T}$ of $\mathbb{G}$ is a tangent subgroup to graph $(\phi)$ in 0 if

(1) $\mathbb{T}$ is a complementary subgroup of $\mathbb{M}$

(2) in any compact subset of $\mathbb{G}$

$$
\lim _{\lambda \rightarrow \infty} \delta_{\lambda}(\operatorname{graph}(\phi))=\mathbb{T}
$$

in the sense of Hausdorff convergence.

Moreover in [10], Theorem 3.2.8 the authors show that $\phi$ is intrinsic differentiable in 0 if and only if graph $(\phi)$ has a tangent subgroup $\mathbb{T}$ in 0 and in this case $\mathbb{T}=\operatorname{graph}\left(d \phi_{0}\right)$.

In addition to pointwise intrinsic differentiability, we are interested in an appropriate notion of continuously intrinsic differentiable functions. For functions acting between complementary subgroups, one possible way is to introduce a stronger, i.e. uniform, notion of intrinsic differentiability in the general setting of Definition 3.2.

Definition 3.3. Let $\mathbb{W}$ and $\mathbb{M}$ be complementary subgroups in $\mathbb{G}$ and $\phi: \mathcal{O} \subset \mathbb{W} \rightarrow \mathbb{M}$ with $\mathcal{O}$ open in $\mathbb{W}$. For any $A \in \mathcal{O}$, let $P:=A \cdot \phi(A)$ and $\phi_{P^{-1}}: \mathcal{O}_{P^{-1}} \subset \mathbb{W} \rightarrow \mathbb{M}$ be the shifted function defined in Proposition 2.5. We say that $\phi$ is uniformly intrinsic differentiable in $A_{0} \in \mathcal{O}$ or $\phi$ is u.i.d. in $A_{0}$ if there exist a intrinsic linear function $d \phi_{A_{0}}: \mathbb{W} \rightarrow \mathbb{M}$ such that

$$
\lim _{r \rightarrow 0^{+}} \sup _{\left\|A_{0}^{-1} A\right\|<r} \sup _{0<\|B\|<r} \frac{\left\|d \phi_{A_{0}}(B)^{-1} \phi_{P^{-1}}(B)\right\|}{\|B\|}=0 .
$$

Analogously, $\phi$ is u.i.d. in $\mathcal{O}$ if it is u.i.d. in every point of $\mathcal{O}$.

Remark 3.3. We recall that in Definition 3.16 in [3] the authors give another notion of uniformly intrinsic differentiable map. It is possible compare these two notions; indeed, for example, Proposition 3.7 (3) is in the definition of u.i.d. for [3]. Moreover, it is clear, taking $A=A_{0}$ in (8), that if $\phi$ is uniformly intrinsic differentiable in $A_{0}$ then it is intrinsic differentiable in $A_{0}$ and $d \phi_{A_{0}}$ is the intrinsic differential of $\phi$ at $A_{0}$ (that is the first point of the definition for [3]). 
From now on we restrict our setting studying the notions of intrinsic differentiability and of uniform intrinsic differentiability for functions $\phi: \mathbb{W} \rightarrow \mathbb{H}$ when $\mathbb{H}$ is a horizontal subgroup. When $\mathbb{H}$ is horizontal, $\mathbb{W}$ is always a normal subgroup since, as observed in Remark 2.3 , it contains the whole strata $\mathbb{G}^{2}, \ldots, \mathbb{G}^{\kappa}$. In this case, the more explicit form of the shifted function $\phi_{P^{-1}}$ allows a more explicit form of equations (77) and (8).

First we observe that, when the target space is horizontal, intrinsic linear functions are Euclidean linear functions from the first layer of $\mathbb{W}$ to $\mathbb{H}$. The analogous of the following proposition is Proposition 3.23, 3] in the Heisenberg groups.

Proposition 3.4. Let $\mathbb{W}$ and $\mathbb{H}$ be complementary subgroups in $\mathbb{G}$ with $\mathbb{H}$ horizontal. Then a intrinsic linear function $\ell: \mathbb{W} \rightarrow \mathbb{H}$ depends only on the variables in the first layer $\mathbb{W}^{1}:=\mathbb{W} \cap \mathbb{G}^{1}$ of $\mathbb{W}$. That is

$$
\ell(A)=\ell\left(A^{1}, 0, \ldots, 0\right), \quad \text { for all } A=\left(A^{1}, \ldots, A^{\kappa}\right) \in \mathbb{W} .
$$

Moreover there is $C_{L} \geq 0$ such that, for all $A \in \mathbb{W}$,

$$
\|\ell(A)\| \leq C_{L}\left\|\left(A^{1}, 0, \ldots, 0\right)\right\|
$$

and $\ell_{\mathbb{W}^{1}}: \mathbb{W}^{1} \rightarrow \mathbb{H}$ is Euclidean linear.

Finally if $k<m_{1}$ is the dimension of $\mathbb{H}$ and if, without loss of generality, we assume that

$$
\mathbb{H}=\left\{P=\left(p_{1}, \ldots, p_{N}\right): p_{k+1}=\cdots=p_{N}=0\right\}, \quad \mathbb{W}=\left\{P=\left(p_{1}, \ldots, p_{N}\right): p_{1}=\cdots=p_{k}=0\right\}
$$

then there is a $k \times\left(m_{1}-k\right)$ matrix $\mathcal{L}$ such that

$$
\ell(A)=\left(\mathcal{L}\left(a_{k+1}, \ldots, a_{m_{1}}\right)^{T}, 0, \ldots, 0\right)
$$

for all $A=\left(a_{1}, \ldots, a_{N}\right) \in \mathbb{W}$.

Proof. In order to prove (9) first we prove that for all $P=\left(P^{1}, \ldots, P^{\kappa}\right) \in \mathbb{W}$, where $P^{i} \in \mathbb{R}^{n_{i}}$,

$$
\ell\left(P^{1}, \ldots, P^{\kappa}\right)=\ell\left(P^{1}, \ldots, P^{\kappa-1}, 0\right) .
$$

Let $A_{\kappa}:=\left(0, \ldots, 0, P^{\kappa}\right) \in \mathbb{W}$. From (2) we have $\ell\left(A_{\kappa}\right)^{-1} \cdot A_{\kappa} \cdot \ell\left(A_{\kappa}\right)=A_{\kappa}$ and by (6)

$$
\ell\left(A_{\kappa} \cdot A_{\kappa}\right)=\ell\left(A_{\kappa}\right) \cdot \ell\left(\ell\left(A_{\kappa}\right)^{-1} \cdot A_{\kappa} \cdot \ell\left(A_{\kappa}\right)\right)=\ell\left(A_{\kappa}\right) \cdot \ell\left(A_{\kappa}\right)=2 \ell\left(A_{\kappa}\right) .
$$

Because $A_{\kappa} \cdot A_{\kappa}=\left(0, \ldots, 0,2 P^{\kappa}\right)=\delta_{2^{1 / \kappa}} A_{\kappa}$, then $2 \ell\left(A_{\kappa}\right)=\ell\left(A_{\kappa} \cdot A_{\kappa}\right)=\ell\left(\delta_{2^{1 / \kappa}} A_{\kappa}\right)=2^{1 / \kappa} \ell\left(A_{\kappa}\right)$; hence

$$
\ell\left(A_{\kappa}\right)=0 .
$$

Because $\left(P^{1}, \ldots, P^{\kappa}\right)=A_{\kappa} \cdot\left(P^{1}, \ldots, P^{\kappa-1}, 0\right)$, from (66) and (13) we get

$$
\begin{aligned}
\ell\left(P^{1}, \ldots, P^{\kappa}\right) & =\ell\left(A_{\kappa} \cdot\left(P^{1}, \ldots, P^{\kappa-1}, 0\right)\right)=\ell\left(A_{\kappa}\right) \cdot \ell\left(\ell\left(A_{\kappa}\right)^{-1} \cdot\left(P^{1}, \ldots, P^{\kappa-1}, 0\right) \cdot \ell\left(A_{\kappa}\right)\right) \\
& =\ell\left(P^{1}, \ldots, P^{\kappa-1}, 0\right),
\end{aligned}
$$

and (12) is proved.

In the next step we prove that

$$
\ell\left(P^{1}, \ldots, P^{\kappa}\right)=\ell\left(P^{1}, \ldots, P^{\kappa-2}, 0,0\right)
$$

Let $A_{\kappa-1}:=\left(0, \ldots, 0, P^{\kappa-1}, 0\right) \in \mathbb{W}$. From (3) , there is $\hat{P}^{\kappa}$, depending on $\ell\left(A_{\kappa-1}\right)$ and $A_{\kappa-1}$, such that

$$
\ell\left(A_{\kappa-1}\right)^{-1} \cdot A_{\kappa-1} \cdot \ell\left(A_{\kappa-1}\right)=\left(0, \ldots, 0, P^{\kappa-1}, \hat{P}^{\kappa}\right)
$$


From (66), (13) and the fact that $\left(0, \ldots, 0, P^{\kappa-1}, \hat{P}^{\kappa}\right)=\left(0, \ldots, 0, \hat{P}^{\kappa}\right) \cdot A_{\kappa-1}$ we get

$$
\begin{aligned}
\ell\left(0, \ldots, 0, P^{\kappa-1}, \hat{P}^{\kappa}\right) & =\ell\left(\left(0, \ldots, 0, \hat{P}^{\kappa}\right) \cdot A_{\kappa-1}\right) \\
& =\ell\left(0, \ldots, 0, \hat{P}^{\kappa}\right) \cdot \ell\left(\ell\left(0, \ldots, 0, \hat{P}^{\kappa}\right)^{-1} \cdot A_{\kappa-1} \cdot \ell\left(0, \ldots, 0, \hat{P}^{\kappa}\right)\right) \\
& =\ell\left(A_{\kappa-1}\right)
\end{aligned}
$$

and consequently

$$
\ell\left(A_{\kappa-1} \cdot A_{\kappa-1}\right)=\ell\left(A_{\kappa-1}\right) \cdot \ell\left(\ell\left(A_{\kappa-1}\right)^{-1} \cdot A_{\kappa-1} \cdot \ell\left(A_{\kappa-1}\right)\right)=\ell\left(A_{\kappa-1}\right) \cdot \ell\left(A_{\kappa-1}\right) .
$$

Because $A_{\kappa-1} \cdot A_{\kappa-1}=\left(0, \ldots, 0,2 P^{\kappa-1}, 0\right)=\delta_{2^{1 /(\kappa-1)}} A_{\kappa-1}$ we have

$$
2 \ell\left(A_{\kappa-1}\right)=\ell\left(A_{\kappa-1}\right) \cdot \ell\left(A_{\kappa-1}\right)=\ell\left(A_{\kappa-1} \cdot A_{\kappa-1}\right)=\ell\left(\delta_{2^{1 /(\kappa-1)}} A_{\kappa-1}\right)=2^{1 /(\kappa-1)} \ell\left(A_{\kappa-1}\right) .
$$

Then

$$
\ell\left(A_{\kappa-1}\right)=\ell\left(0, \ldots, 0, P^{\kappa-1}, \hat{P}^{\kappa}\right)=0 .
$$

Because $\left(P^{1}, \ldots, P^{\kappa}\right)=\left(P^{1}, \ldots, P^{\kappa-2}, 0,0\right) \cdot\left(0, \ldots, 0, P^{\kappa-1}, \bar{P}^{\kappa}\right)$ for appropriate $\bar{P}^{\kappa}$ and $\ell\left(0, \ldots, 0, P^{\kappa-1}, \bar{P}^{\kappa}\right)=0$, 11 we obtain (14) from (6). This procedure can be iterated to get (9)).

Now it is easy to see that $\ell$ is Euclidean linear. Indeed for all $A, B \in \mathbb{W}$ and $\lambda>0$

$$
\ell\left(\left(\lambda A^{1}, 0 \ldots, 0\right)\right)=\ell\left(\left(\delta_{\lambda} A\right)^{1}, 0 \ldots, 0\right)=\ell\left(\delta_{\lambda} A\right)=\delta_{\lambda} \ell(A)=\lambda \ell\left(A^{1}, 0 \ldots, 0\right)
$$

and

$$
\begin{aligned}
& \ell\left(\left(A^{1}, 0 \ldots, 0\right)+\left(B^{1}, 0 \ldots, 0\right)\right)=\ell\left((A B)^{1}, 0 \ldots, 0\right)=\ell(A B) \\
& =\ell(A) \ell\left(\ell(A)^{-1} B \ell(A)\right) \\
& \left.=\ell\left(A^{1}, 0 \ldots, 0\right) \ell\left(\left(\ell(A)^{-1} B \ell(A)\right)^{1}, 0, \ldots, 0\right)\right) \\
& =\ell\left(A^{1}, 0 \ldots, 0\right) \ell\left(B^{1}, 0, \ldots, 0\right)=\ell\left(A^{1}, 0 \ldots, 0\right)+\ell\left(B^{1}, 0, \ldots, 0\right) .
\end{aligned}
$$

Keeping in mind this special form of intrinsic linear functions we obtain the following special form of intrinsic differentiability. The reader can see Proposition 3.25 (ii) and Proposition 3.26 (ii) in [3] for the Heisenberg groups.

Proposition 3.5. Let $\mathbb{H}$ and $\mathbb{W}$ be complementary subgroups of $\mathbb{G}, \mathcal{O}$ open in $\mathbb{W}$ and $\mathbb{H}$ horizontal. Then $\phi: \mathcal{O} \subset \mathbb{W} \rightarrow \mathbb{H}$ is intrinsic differentiable in $A_{0} \in \mathcal{O}$ if and only if there is a intrinsic linear $d \phi_{A_{0}}: \mathbb{W} \rightarrow \mathbb{H}$ such that

$$
\lim _{r \rightarrow 0^{+}} \sup _{0<\left\|A_{0}^{-1} B\right\|<r} \frac{\left\|\phi(B)-\phi\left(A_{0}\right)-d \phi_{A_{0}}\left(A_{0}^{-1} B\right)\right\|}{\left\|\phi\left(A_{0}\right)^{-1} A_{0}^{-1} B \phi\left(A_{0}\right)\right\|}=0 .
$$

Analogously, $\phi$ is uniformly intrinsic differentiable in $A_{0} \in \mathcal{O}$ if there is a intrinsic linear $d \phi_{A_{0}}$ : $\mathbb{W} \rightarrow \mathbb{H}$ such that

$$
\lim _{r \rightarrow 0^{+}} \sup _{\left\|A_{0}^{-1} A\right\|<r} \sup _{0<\left\|A^{-1} B\right\|<r} \frac{\left\|\phi(B)-\phi(A)-d \phi_{A_{0}}\left(A^{-1} B\right)\right\|}{\left\|\phi(A)^{-1} A^{-1} B \phi(A)\right\|}=0
$$

where $r$ is small enough so that $\mathcal{U}\left(A_{0}, 2 r\right) \subset \mathcal{O}$.

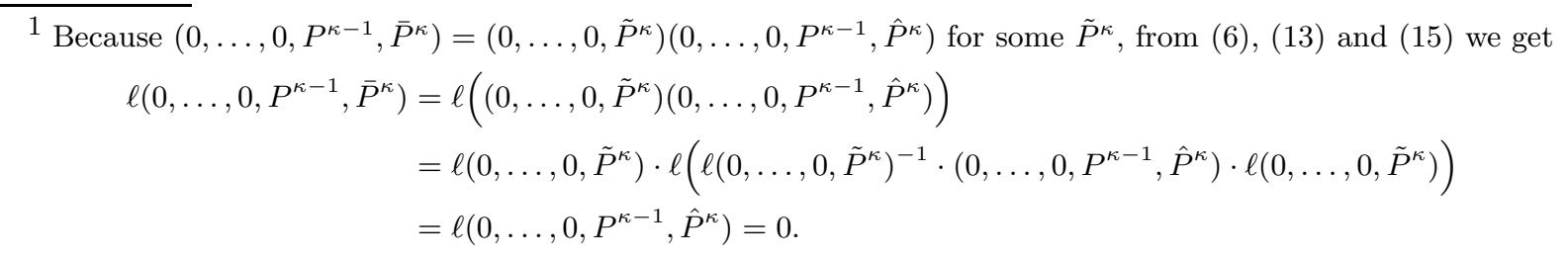


Proof. First notice that $\phi(B)-\phi(A)-d \phi_{A_{0}}\left(A^{-1} B\right)=d \phi_{A_{0}}\left(A^{-1} B\right)^{-1} \phi(A)^{-1} \phi(B)$ because both $d \phi_{A_{0}}$ and $\phi$ are valued in the horizontal subgroup $\mathbb{H}$. Because $\mathbb{W}$ is normal in $\mathbb{G}$, then for $P:=A \phi(A)$ and for all $B^{\prime} \in \mathcal{O}_{P^{-1}}$

$$
\phi_{P^{-1}}\left(B^{\prime}\right)=\phi(A)^{-1} \phi\left(A \phi(A) B^{\prime} \phi(A)^{-1}\right) .
$$

Then (77) yields that $\phi: \mathcal{O} \rightarrow \mathbb{H}$ is intrinsic differentiable in $A_{0} \in \mathcal{O}$ if there is an intrinsic linear $\operatorname{map} d \phi_{A_{0}}: \mathbb{W} \rightarrow \mathbb{H}$ such that

$$
\left\|d \phi_{A_{0}}\left(B^{\prime}\right)^{-1} \phi(A)^{-1} \phi\left(A \phi(A) B^{\prime} \phi(A)^{-1}\right)\right\|=o\left(\left\|B^{\prime}\right\|\right) \quad \text { as }\left\|B^{\prime}\right\| \rightarrow 0,
$$

that, setting $B:=A \phi(A) B^{\prime} \phi(A)^{-1}$ is equivalent to

$$
\left\|d \phi_{A_{0}}\left(\phi(A)^{-1} A^{-1} B \phi(A)\right)^{-1} \phi(A)^{-1} \phi(B)\right\|=o\left(\left\|\phi(A)^{-1} A^{-1} B \phi(A)\right\|\right) \quad \text { as }\left\|A^{-1} B\right\| \rightarrow 0 .
$$

Finally, from Proposition 3.4 we know that $d \phi_{A_{0}}$ depends only on the variables in the first layer of $\mathbb{W}$. The group operation on the first layer is commutative hence $d \phi_{A_{0}}\left(\phi(A)^{-1} A^{-1} B \phi(A)\right)=$ $d \phi_{A_{0}}\left(A^{-1} B\right)$.

Remark 3.6. If $k<m_{1}$ is the dimension of $\mathbb{H}$, and if, without loss of generality, we assume that

$$
\mathbb{H}=\left\{P: p_{k+1}=\cdots=p_{N}=0\right\} \quad \mathbb{W}=\left\{P: p_{1}=\cdots=p_{k}=0\right\}
$$

then, from (11), there is a $k \times\left(m_{1}-k\right)$ matrix, here denoted as $\nabla^{\phi} \phi\left(A_{0}\right)$, such that

$$
d \phi_{A_{0}}(B)=\left(\nabla^{\phi} \phi\left(A_{0}\right)\left(b_{k+1}, \ldots, b_{m_{1}}\right)^{T}, 0, \ldots, 0\right),
$$

for all $B=\left(b_{1}, \ldots, b_{N}\right) \in \mathbb{W}$. The matrix $\nabla^{\phi} \phi\left(A_{0}\right)$ is called the intrinsic horizontal Jacobian of $\phi$ in $A_{0}$ or the intrinsic horizontal gradient or even the intrinsic gradient if $k=1$.

The point (1) of the following proposition states precisely a natural relation between uniform intrinsic differentiability and intrinsic Lipschitz continuity (see Proposition 3.30 [3] for the Heisenberg groups). The point (2) is a generalization of what was previously known for u.i.d. functions in Heisenberg groups (see [2], Theorem 1.3).

Proposition 3.7. Let $\mathbb{H}, \mathbb{W}$ be complementary subgroups of $\mathbb{G}$ with $\mathbb{H}$ horizontal. Let $\mathcal{O}$ be open in $\mathbb{W}$ and $\phi: \mathcal{O} \rightarrow \mathbb{H}$ be u.i.d. in $\mathcal{O}$. Then

(1) $\phi$ is intrinsic Lipschitz continuous in every relatively compact subset of $\mathcal{O}$.

(2) $\phi \in h_{\text {loc }}^{1 / \kappa}(\mathcal{O})$, that is $\phi \in \mathbb{C}(\mathcal{O}, \mathbb{R})$ and for all $\mathcal{F} \Subset \mathcal{O}$ and $A, B \in \mathcal{F}$

$$
\lim _{r \rightarrow 0^{+}} \sup _{0<\left\|A^{-1} B\right\|<r} \frac{\|\phi(B)-\phi(A)\|}{\left\|A^{-1} B\right\|^{1 / \kappa}}=0 .
$$

(3) the function $A \mapsto d \phi_{A}$ is continuous in $\mathcal{O}$.

Proof. (Proof of 1) For each $A_{0} \in \mathcal{O}$ there is $r=r\left(A_{0}\right)>0$ s.t. for all $A, B \in \mathcal{U}\left(A_{0}, r\right) \cap \mathcal{O}$

$$
\left\|\phi(B)-\phi(A)-d \phi_{A_{0}}\left(A^{-1} B\right)\right\| \leq\left\|\phi(A)^{-1} A^{-1} B \phi(A)\right\| .
$$

Moreover from Proposition [3.4, because the intrinsic linear function $d \phi_{A_{0}}$ depends only on the variables on the first layer of $\mathbb{W}$, we have

$$
\left\|d \phi_{A_{0}}\left(A^{-1} B\right)\right\| \leq C_{L}\left|\left(A^{-1} B\right)^{1}\right|_{\mathbb{R}^{m_{1}}} \leq C_{L}\left\|\phi(A)^{-1} A^{-1} B \phi(A)\right\|,
$$

where $C_{L}$ is the intrinsic Lipschitz constant of $d \phi_{A_{0}}$. Finally

$$
\begin{aligned}
\|\phi(B)-\phi(A)\| & \leq\left\|\phi(B)-\phi(A)-d \phi_{A_{0}}\left(A^{-1} B\right)\right\|+\left\|d \phi_{A_{0}}\left(A^{-1} B\right)\right\| \\
& \leq\left(1+C_{L}\right)\left\|\phi(A)^{-1} A^{-1} B \phi(A)\right\| .
\end{aligned}
$$

Then (1) follows by a standard covering argument. 
(Proof of 2) For $A_{0} \in \mathcal{O}, A, B \in \mathcal{U}\left(A_{0}, r\right) \cap \mathcal{O}$ and $r>0$ let

$$
\rho(r):=\sup _{0<\left\|A^{-1} B\right\|<r} \frac{\left\|\phi(B)-\phi(A)-d \phi_{A_{0}}\left(A^{-1} B\right)\right\|}{\left\|\phi(A)^{-1} A^{-1} B \phi(A)\right\|}
$$

then $\lim _{r \rightarrow 0} \rho(r)=0$ because $\phi$ is u.i.d. at $A_{0}$. Moreover, by (1) of this Proposition, we know that $\phi$ is intrinsic Lipschitz in $\mathcal{U}\left(A_{0}, r\right) \cap \mathcal{O}$ and by Proposition 2.7 (1) we have that $\|\phi(A)\|<C_{1}$ for all $A \in \mathcal{U}\left(A_{0}, r\right) \cap \mathcal{O}$.

We recall (see [11, Lemma 2.2.10) that in any Carnot group $\mathbb{G}$ of step $\kappa$ there is $C=C(\mathbb{G})>0$ such that

$$
\left\|Q^{-1} P Q\right\| \leq\|P\|+C\left(\|P\|^{\frac{1}{\kappa}}\|Q\|^{\frac{\kappa-1}{\kappa}}+\|P\|^{\frac{\kappa-1}{\kappa}}\|Q\|^{\frac{1}{\kappa}}\right) \quad \text { for all } P, Q \in \mathbb{G} .
$$

From (18) with $P=A^{-1} B$ and $Q=\phi(A)$ we deduce the existence of $C=C\left(C_{1}\right)>0$ such that

$$
\left\|\phi(A)^{-1} A^{-1} B \phi(A)\right\| \leq C\left\|A^{-1} B\right\|^{1 / \kappa} \quad \text { for all } A, B \in \mathcal{U}\left(A_{0}, r\right) \cap \mathcal{O} .
$$

Therefore using (10)

$$
\begin{aligned}
& \frac{\|\phi(B)-\phi(A)\|}{\left\|A^{-1} B\right\|^{1 / \kappa}} \\
& \leq \frac{\left\|\phi(B)-\phi(A)-d \phi_{A_{0}}\left(A^{-1} B\right)\right\|}{\left\|\phi(A)^{-1} A^{-1} B \phi(A)\right\|} \frac{\left\|\phi(A)^{-1} A^{-1} B \phi(A)\right\|}{\left\|A^{-1} B\right\|^{1 / \kappa}}+\frac{\left\|d \phi_{A_{0}}\left(A^{-1} B\right)\right\|}{\left\|A^{-1} B\right\|^{1 / \kappa}} \\
& \leq C \rho(r)+C_{2} r^{1-1 / \kappa}
\end{aligned}
$$

for all $A, B \in \mathcal{U}\left(A_{0}, r\right) \cap \mathcal{O}$ with $A \neq B$. Hence $\frac{\|\phi(B)-\phi(A)\|}{\left\|A^{-1} B\right\|^{1 / \kappa}} \rightarrow 0$ for $r \rightarrow 0$ and the proof of (2) is complete.

(Proof of 3) In order to prove that $A \mapsto d \phi_{A}$ is continuous in $A_{0} \in \mathcal{O}$ we prove that for all $\epsilon>0$ there is $r=r\left(\epsilon, A_{0}\right)>0$ such that

$$
\left\|d \phi_{A_{1}}(P)-d \phi_{A_{0}}(P)\right\|<\epsilon\|P\|
$$

for $A_{1} \in \mathcal{U}\left(A_{0}, r\right) \cap \mathbb{W}$ and for all $P \in \mathbb{W}$.

Indeed, because $\phi$ is u.i.d. in $A_{0}$, for all $\epsilon>0$ there is $r_{0}=r_{0}\left(\epsilon, A_{0}\right)>0$ such that

$$
\left\|\phi(B)-\phi(A)-d \phi_{A_{0}}\left(A^{-1} B\right)\right\|<\epsilon\left\|\phi(A)^{-1} A^{-1} B \phi(A)\right\| \quad \text { for all } A, B \in \mathcal{U}\left(A_{0}, r_{0}\right) \cap \mathbb{W} \text {. }
$$

Let $A_{1} \in \mathcal{U}\left(A_{0}, r_{0}\right) \cap \mathbb{W}$. By assumption $\phi$ is u.i.d. also in $A_{1}$ hence there is $r_{1}=r_{1}\left(\epsilon, A_{1}\right)>0$ such that $\mathcal{U}\left(A_{1}, r_{1}\right) \subset \mathcal{U}\left(A_{0}, r_{0}\right)$ and

$$
\left\|\phi(B)-\phi(A)-d \phi_{A_{1}}\left(A^{-1} B\right)\right\|<\epsilon\left\|\phi(A)^{-1} A^{-1} B \phi(A)\right\| \quad \text { for all } A, B \in \mathcal{U}\left(A_{1}, r_{1}\right) \cap \mathbb{W} .
$$

Hence

$$
\left\|d \phi_{A_{1}}\left(A^{-1} B\right)-d \phi_{A_{0}}\left(A^{-1} B\right)\right\|<2 \epsilon\left\|\phi(A)^{-1} A^{-1} B \phi(A)\right\| \quad \text { for all } A, B \in \mathcal{U}\left(A_{1}, r_{1}\right) \cap \mathbb{W} .
$$

Let $\delta \in(0,1)$ be a constant to be chosen at the end. For all $P \in \mathcal{U}\left(0, \delta r_{1}\right) \cap \mathbb{W}$ let $B:=A_{1} P$. Then $B \in \mathcal{U}\left(A_{1}, r_{1}\right)$ and

$$
\left\|d \phi_{A_{1}}(P)-d \phi_{A_{0}}(P)\right\|<2 \epsilon\left\|\phi\left(A_{1}\right)^{-1} P \phi\left(A_{1}\right)\right\| .
$$

The proof is completed if we can put on the right hand side $\|P\|$ instead of $\left\|\phi\left(A_{1}\right)^{-1} P \phi\left(A_{1}\right)\right\|$.

Observe that $d \phi_{A_{1}}$ and $d \phi_{A_{0}}$ depend only on the components in the first layer of $\mathbb{W}$. Consequently we can change the components $P^{2}, \ldots, P^{\kappa}$ of $P$ without changing $d \phi_{A_{1}}(P)-d \phi_{A_{0}}(P)$.

An explicit computation shows that

$$
\phi\left(A_{1}\right)^{-1} P \phi\left(A_{1}\right)=\left(P^{1}, P^{2}+\mathcal{P}^{2}\left(P^{1}, \phi\left(A_{1}\right)\right), \ldots, P^{\kappa}+\mathcal{P}^{\kappa}\left(P^{1}, \ldots, P^{\kappa-1}, \phi\left(A_{1}\right)\right)\right)
$$

where $\mathcal{P}^{2}, \ldots, \mathcal{P}^{\kappa}$ are polynomials. Moreover each polynomial $\mathcal{P}^{i}$ depends only on the variables in the layers from 1 to $i-1$. 
Now we can conclude. Given $P=\left(P^{1}, \ldots, P^{\kappa}\right) \in \mathcal{U}\left(0, \delta r_{1}\right) \cap \mathbb{W}$, we define $\tilde{P}=\left(\tilde{P}^{1}, \ldots, \tilde{P}^{\kappa}\right)$ putting iteratively

$$
\begin{aligned}
& \tilde{P}^{1}:=P^{1} \\
& \tilde{P}^{2}:=-\mathcal{P}^{2}\left(\tilde{P}^{1}, \phi\left(A_{1}\right)\right) \\
& \ldots \\
& \tilde{P}^{\kappa}:=-\mathcal{P}^{\kappa}\left(\tilde{P}^{1}, \ldots, \tilde{P}^{\kappa-1}, \phi\left(A_{1}\right)\right) .
\end{aligned}
$$

Observe that, if $\delta$ is sufficiently small, $\tilde{P} \in \mathcal{U}\left(0, r_{1}\right)$, moreover $\phi\left(A_{1}\right)^{-1} \tilde{P} \phi\left(A_{1}\right)=\left(\tilde{P}^{1}, 0, \ldots, 0\right)$ and $\left\|\phi\left(A_{1}\right)^{-1} \tilde{P} \phi\left(A_{1}\right)\right\|=\left|\tilde{P}^{1}\right|=\left|P^{1}\right|$. Finally

$$
\left\|d \phi_{A_{1}}(P)-d \phi_{A_{0}}(P)\right\|=\left\|d \phi_{A_{1}}(\tilde{P})-d \phi_{A_{0}}(\tilde{P})\right\| \leq 2 \epsilon\left|P^{1}\right|_{\mathbb{R}^{m_{1}}}
$$

for all $P \in \mathcal{U}\left(0, \delta r_{1}\right) \cap \mathbb{W}$. By Proposition 3.1, it holds for all $P \in \mathbb{W}$ and the proof is completed.

\section{4. $\mathbb{G}$-REgUlaR SURFACES}

The main result of this section and of the first part of this paper is Theorem 4.1. In it we prove that, if $\mathbb{H}$ is a horizontal subgroup, the intrinsic graph of $\phi: \mathcal{O} \subset \mathbb{W} \rightarrow \mathbb{H}$ is a $\mathbb{G}$-regular $k$-codimensional surface if and only if $\phi$ is uniformly intrinsic differentiable in $\mathcal{O}$.

Theorem 4.1. Let $\mathbb{W}$ and $\mathbb{H}$ be complementary subgroups of a Carnot group $\mathbb{G}$ with $\mathbb{H}$ horizontal and $k$ dimensional. Let $\mathcal{O}$ be open in $\mathbb{W}, \phi: \mathcal{O} \subset \mathbb{W} \rightarrow \mathbb{H}$ and $S:=$ graph $(\phi)$. Then for every $A_{0} \in \mathcal{O}$ the following are equivalent:

(1) there are a neighbourhood $\mathcal{U}$ of $A_{0} \cdot \phi\left(A_{0}\right)$ and $f \in \mathbb{C}_{\mathbb{G}}^{1}\left(\mathcal{U} ; \mathbb{R}^{k}\right)$ such that

$$
\begin{aligned}
& S \cap \mathcal{U}=\{P \in \mathcal{U}: f(P)=0\} \\
& d_{\mathbf{P}} f(Q)_{\mid \mathbb{H}}: \mathbb{H} \rightarrow \mathbb{R}^{k} \quad \text { is bijective for all } Q \in \mathcal{U}
\end{aligned}
$$

and $Q \mapsto\left(d_{\mathbf{P}} f(Q)_{\mathbb{H}}\right)^{-1}$ is continuous.

(2) $\phi$ is u.i.d. in a neighbourhood $\mathcal{O}^{\prime} \subset \mathcal{O}$ of $A_{0}$.

Moreover, if (1) or equivalently (2), hold then, for all $A \in \mathcal{O}$ the intrinsic differential $d \phi_{A}$ is

$$
d \phi_{A}=-\left(d_{\mathbf{P}} f(A \phi(A))_{\mid \mathbb{H}}\right)^{-1} \circ d_{\mathbf{P}} f(A \phi(A))_{\mid \mathbb{W}} .
$$

Remark 4.2. If, without loss of generality, we choose a base $X_{1}, \ldots, X_{N}$ of $\mathfrak{g}$ such that $X_{1}, \ldots, X_{k}$ are horizontal vector fields, $\mathbb{H}=\exp \left(\operatorname{span}\left\{X_{1}, \ldots, X_{k}\right\}\right)$ and $\mathbb{W}=\exp \left(\operatorname{span}\left\{X_{k+1}, \ldots, X_{N}\right\}\right)$ then

$$
\mathbb{H}=\left\{P: p_{k+1}=\cdots=p_{N}=0\right\} \quad \mathbb{W}=\left\{P: p_{1}=\cdots=p_{k}=0\right\},
$$

and, if $f=\left(f_{1}, \ldots, f_{k}\right)$, then $\nabla_{\mathbb{G}} f=\left(\mathcal{M}_{1} \mid \mathcal{M}_{2}\right)$ where

$$
\mathcal{M}_{1}:=\left(\begin{array}{llr}
X_{1} f_{1} \ldots & X_{k} f_{1} \\
\vdots & \ddots & \vdots \\
X_{1} f_{k} \ldots & \ldots X_{k} f_{k}
\end{array}\right), \quad \mathcal{M}_{2}:=\left(\begin{array}{ccc}
X_{k+1} f_{1} & \ldots & X_{m_{1}} f_{1} \\
\vdots & \ddots & \vdots \\
X_{k+1} f_{k} & \ldots & X_{m_{1}} f_{k}
\end{array}\right) .
$$

Moreover, for all $Q \in \mathcal{U}$, for all $A \in \mathcal{O}$ and for all $P \in \mathbb{G}$

$$
\left(d_{\mathbf{P}} f(Q)\right)(P)=\left(\nabla_{\mathbb{G}} f(Q)\right) P^{1}
$$

and the intrinsic differential is

$$
\begin{aligned}
d \phi_{A}(B) & =\left(\left(\nabla^{\phi} \phi(A)\right)\left(b_{k+1}, \ldots, b_{m_{1}}\right)^{T}, 0, \ldots, 0\right) \\
& =\left(\left(-\mathcal{M}_{1}(A \phi(A))^{-1} \mathcal{M}_{2}(A \phi(A))\right)\left(b_{k+1}, \ldots, b_{m_{1}}\right)^{T}, 0, \ldots, 0\right),
\end{aligned}
$$


for all $B=\left(b_{1}, \ldots, b_{N}\right) \in \mathbb{W}($ see (16) $)$.

The proof of Theorem 4.1 requires both Whitney's Extension Theorem and Implicit Function Theorem in Carnot groups. The proof of Whitney's Extension Theorem can be found in [15] for Carnot groups of step two only, but it is identical for general Carnot groups (see [7], Theorem 2.3.8).

Theorem 4.3 (Implicit Function Theorem, see [22], Theorem 1.3). Let $\mathcal{U}$ be an open subset of $\mathbb{G}$. Let $f \in \mathbb{C}_{\mathbb{G}}^{1}\left(\mathcal{U}, \mathbb{R}^{k}\right)$ and assume that $\nabla_{\mathbb{G}} f(Q)$ has rank $k$ for all $Q \in \mathcal{U}$. We assume that for a fixed $P \in \mathcal{U}$ there are complementary subgroups $\mathbb{W}$ and $\mathbb{H}$ of $\mathbb{G}$ where $\mathbb{W}=k \operatorname{er}\left(\nabla_{\mathbb{G}} f(P)\right)$. Then there are $\mathcal{I} \subset \mathbb{W}$ and $\mathcal{J} \subset \mathbb{H}$, open and such that $P_{\mathbb{W}} \in \mathcal{I}$ and $P_{\mathbb{H}} \in \mathcal{J}$ and a unique continuous function $\phi: \mathcal{I} \rightarrow \mathcal{J}$ such that

$$
\{Q \in \mathcal{I} \mathcal{J}: f(Q)=f(P)\}=\{A \phi(A): A \in \mathcal{I}\}
$$

where $\mathcal{I} \mathcal{J}=\{A B: A \in \mathcal{I}, B \in \mathcal{J}\}$.

Theorem 4.4 (Whitney's Extension Theorem). Let $\mathcal{F} \subset \mathbb{G}$ be a closed set and let $f$ and $g$ be continuous functions where $f: \mathcal{F} \rightarrow \mathbb{R}^{k}$ and $g: \mathcal{F} \rightarrow \mathbf{M}_{k \times m_{1}}$ the space of $k \times m_{1}$ matrices. For $\mathcal{K} \subset \mathcal{F}, P$ and $Q \in \mathcal{K}, \delta>0$ let

$$
\rho_{\mathcal{K}}(\delta):=\sup _{0<\left\|Q^{-1} P\right\|<\delta} \frac{\left|f(P)-f(Q)-g(Q)\left(Q^{-1} P\right)^{1}\right|_{\mathbb{R}^{k}}}{\left\|Q^{-1} P\right\|}
$$

where $g(Q)\left(Q^{-1} P\right)^{1}$ is the usual product between matrix and vector. If, for all compact set $\mathcal{K} \subset \mathcal{F}$,

$$
\lim _{\delta \rightarrow 0} \rho_{\mathcal{K}}(\delta)=0
$$

then there exists $\hat{f} \in \mathbb{C}_{\mathbb{G}}^{1}\left(\mathbb{G}, \mathbb{R}^{k}\right)$ such that

$$
\hat{f}_{\mid \mathcal{F}}=f, \quad \nabla_{\mathbb{G}} \hat{f}_{\mid \mathcal{F}}=g .
$$

A proof is in [15] when $\mathbb{G}$ is a Carnot groups of step two. The general case is in [7].

Before proving Theorem 4.1 we state a Morrey type inequality for functions in $\mathbb{C}_{\mathbb{G}}^{1}\left(\mathbb{G}, \mathbb{R}^{k}\right.$ ) (see also Lemma 3.2.2 in [28]).

Lemma 4.5. Let $P \in \mathbb{G}, r_{0}>0$ and $f \in \mathbb{C}_{\mathbb{G}}^{1}\left(\mathcal{U}\left(P, r_{0}\right), \mathbb{R}^{k}\right)$. Then there is $C=C\left(P, r_{0}\right)>0$ such that, for each $\bar{Q} \in \mathcal{U}\left(P, r_{0} / 2\right)$ and $r \in\left(0, r_{0} / 4\right)$,

$$
\left|f(Q)-f(\bar{Q})-\nabla_{\mathbb{G}} f(\bar{Q})\left(\bar{Q}^{-1} Q\right)^{1}\right|_{\mathbb{R}^{k}} \leq C r\left\|\nabla_{\mathbb{G}} f-\nabla_{\mathbb{G}} f(\bar{Q})\right\|_{\mathcal{L}^{\infty}(\mathcal{U}(\bar{Q}, 2 r))}
$$

for all $Q \in \mathcal{U}(\bar{Q}, r)$.

Proof. Let $\hat{f}: \mathcal{U}(\bar{Q}, r) \rightarrow \mathbb{R}^{k}$ be defined as

$$
\hat{f}(Q):=f(Q)-\nabla_{\mathbb{G}} f(\bar{Q})\left(\bar{Q}^{-1} Q\right)^{1} .
$$

Then (see Theorem 1.1. in [20]) there are $p>1$ and $\hat{C}>0$ such that for all $Q \in \mathcal{U}(\bar{Q}, r)$

$$
|\hat{f}(Q)-\hat{f}(\bar{Q})|_{\mathbb{R}^{k}} \leq \hat{C} r\left(f_{\mathcal{U}(\bar{Q}, 2 r)}\left|\nabla_{\mathbb{G}} \hat{f}\right|^{p} d \mathcal{L}^{N}\right)^{1 / p}
$$


where $f_{\mathcal{U}} \cdot d \mathcal{L}^{N}:=\frac{1}{\mathcal{L}^{N}(\mathcal{U})} \int_{\mathcal{U}} \cdot d \mathcal{L}^{N}$. Then, from $\hat{f}(\bar{Q})=f(\bar{Q})$ and $\nabla_{\mathbb{G}} \hat{f}=\nabla_{\mathbb{G}} f-\nabla_{\mathbb{G}} f(\bar{Q})$, we have

$$
\begin{aligned}
\mid f(Q)- & f(\bar{Q})-\left.\nabla_{\mathbb{G}} f(\bar{Q})\left(\bar{Q}^{-1} Q\right)^{1}\right|_{\mathbb{R}^{k}}=|\hat{f}(Q)-\hat{f}(\bar{Q})|_{\mathbb{R}^{k}} \\
& \leq 2 \hat{C} r\left(f_{\mathcal{U}(\bar{Q}, 2 r)} \mid \nabla_{\mathbb{G}} \hat{f}^{p} d \mathcal{L}^{N}\right)^{1 / p} \\
& =2 \hat{C} r\left(f_{\mathcal{U}(\bar{Q}, 2 r)}\left|\nabla_{\mathbb{G}} f-\nabla_{\mathbb{G}} f(\bar{Q})\right|^{p} d \mathcal{L}^{N}\right)^{1 / p} \\
& \leq C r\left\|\nabla_{\mathbb{G}} f-\nabla_{\mathbb{G}} f(\bar{Q})\right\|_{\mathcal{L}^{\infty}(\mathcal{U}(\bar{Q}, 2 r)) .}
\end{aligned}
$$

Proof of Theorem 4.1. (1) $\Rightarrow(2)$.

Let $A_{0} \in \mathcal{O}, r>0$ such that $\mathcal{I}\left(A_{0}, r\right):=\mathcal{U}\left(A_{0}, r\right) \cap \mathbb{W} \subset \mathcal{O}$ and define $\Phi: \mathcal{O} \rightarrow \mathbb{G}$ as $\Phi(A):=A \phi(A)$ for all $A \in \mathcal{O}$.

The function $\phi$ is continuous in $\mathcal{O}$ as follows from a well known elementary argument in Implicit Function Theorem. Hence there is $\delta_{r}=\delta\left(A_{0}, r\right)>0$ such that $\left\|\Phi(A)^{-1} \cdot \Phi(B)\right\| \leq \delta_{r}$ for all $A, B \in \mathcal{I}\left(A_{0}, r\right)$. Then, recalling that $d_{\mathbf{P}} f=\nabla_{\mathbb{G}} f$ acting on the first layer, for all $A, B \in \mathcal{I}\left(A_{0}, r\right)$

$$
\begin{aligned}
\mid d_{\mathbf{P}} f(\Phi(A)) & \left.\left(\Phi(A)^{-1} \Phi(B)\right)\right|_{\mathbb{R}^{k}} \\
& =\left|f(\Phi(B))-f(\Phi(A))-d_{\mathbf{P}} f(\Phi(A))\left(\Phi(A)^{-1} \Phi(B)\right)\right|_{\mathbb{R}^{k}} \\
& \leq C \rho\left(\delta_{r}\right)\left\|\Phi(A)^{-1} \Phi(B)\right\| \\
& \leq C \rho\left(\delta_{r}\right)\left(\left\|\mathbf{P}_{\mathbb{H}}\left(\Phi(A)^{-1} \Phi(B)\right)\right\|+\left\|\mathbf{P}_{\mathbb{W}}\left(\Phi(A)^{-1} \Phi(B)\right)\right\|\right)
\end{aligned}
$$

where $C$ is the constant in Lemma 4.5, and

$$
\rho\left(\delta_{r}\right):=\left\|\nabla_{\mathbb{G}} f-\nabla_{\mathbb{G}} f\left(\Phi\left(A_{0}\right)\right)\right\|_{\mathcal{L}^{\infty}\left(\mathcal{U}\left(\Phi\left(A_{0}\right), 2 \delta_{r}\right)\right)} .
$$

Observe also that

$$
\lim _{r \rightarrow 0} \rho\left(\delta_{r}\right)=0 .
$$

The function $\phi$, beyond being simply continuous, is also intrinsic Lipschitz continuous i.e. (see Remark 2.6) there is a constant $C_{L}>0$ such that

$$
\left\|\phi(A)^{-1} \cdot \phi(B)\right\| \leq C_{L}\left\|\phi(A)^{-1} A^{-1} B \phi(A)\right\| .
$$

Indeed, because $\mathbf{P}_{\mathbb{H}}\left(\Phi(A)^{-1} \Phi(B)\right)=\phi(A)^{-1} \cdot \phi(B)$ and $\mathbf{P}_{\mathbb{W}}\left(\Phi(A)^{-1} \Phi(B)\right)=\phi(A)^{-1} A^{-1} B \phi(A)$, from (20), we get for all $A, B \in \mathcal{I}\left(A_{0}, \delta_{r}\right)$

$$
\begin{aligned}
& \left\|\left(d_{\mathbf{P}} f(\Phi(A))_{\mathbb{H}}^{-1} \circ d_{\mathbf{P}} f(\Phi(A))_{\mid \mathbb{W}}\right)\left(A^{-1} B\right) \cdot \phi(A)^{-1} \cdot \phi(B)\right\| \\
& =\left\|d_{\mathbf{P}} f(\Phi(A))_{\mathbb{H}}^{-1}\left(d_{\mathbf{P}} f(\Phi(A))_{\mid \mathbb{W}}\left(A^{-1} B\right)+d_{\mathbf{P}} f(\Phi(A))_{\mid \mathbb{H}}\left(\phi(A)^{-1} \cdot \phi(B)\right)\right)\right\|
\end{aligned}
$$

(because $d_{\mathbf{P}} f=\nabla_{\mathbb{G}} f$ which acts only on the first components, $d_{\mathbf{P}} f\left(A^{-1} B\right)=d_{\mathbf{P}} f\left(\phi(A)^{-1} A^{-1} B \phi(A)\right)$ and then we use the fact that $d_{\mathbf{P}} f$ is an homomorphism)

$$
\begin{aligned}
& =\left\|d_{\mathbf{P}} f(\Phi(A))_{\mid \mathbb{H}}^{-1}\left(d_{\mathbf{P}} f(\Phi(A))\left(\Phi(A)^{-1} \cdot \Phi(B)\right)\right)\right\| \\
& \leq C \rho\left(\delta_{r}\right)\left\|d_{\mathbf{P}} f(\Phi(A))_{\mid \mathbb{H}}^{-1}\right\|\left(\left\|\mathbf{P}_{\mathbb{H}}\left(\Phi(A)^{-1} \Phi(B)\right)\right\|+\left\|\mathbf{P}_{\mathbb{W}}\left(\Phi(A)^{-1} \Phi(B)\right)\right\|\right) \\
& \left.=C \rho\left(\delta_{r}\right)\left\|d_{\mathbf{P}} f(\Phi(A))_{\mid \mathbb{H}}^{-1}\right\|\left(\left\|\phi(A)^{-1} \cdot \phi(B)\right\|+\| \phi(A)^{-1} A^{-1} B \phi(A)\right) \|\right) . \\
& 16
\end{aligned}
$$


Now we choose $r$ small enough such that for all $A \in \mathcal{I}\left(A_{0}, r\right)$,

$$
C \rho\left(\delta_{r}\right)\left\|d_{\mathbf{P}} f(\Phi(A))_{\mid \mathbb{H}}^{-1}\right\| \leq \frac{1}{2} .
$$

Moreover, because $d_{\mathbf{P}} f(\Phi(A))_{\mid \mathbb{W}}=d_{\mathbf{P}} f(\Phi(A))_{\mathbb{W}^{1}}$ and the homogeneous norm defined in (44) restricted on the horizontal layer is the Euclidean norm, there is $C_{2}=C_{2}\left(A_{0}, r\right)>0$ such that for all $A, B \in \mathcal{I}\left(A_{0}, \delta_{r}\right)$,

$$
\left\|\left(d_{\mathbf{P}} f(\Phi(A))_{\mid \mathbb{H}}^{-1} \circ d_{\mathbf{P}} f(\Phi(A))_{\mid \mathbb{W}}\right)\left(A^{-1} B\right)\right\| \leq C_{2}\left|\left(A^{-1} B\right)^{1}\right|_{\mathbb{R}^{m_{1}}} .
$$

Putting all this together and by the homogeneous norm defined in (44) is symmetric, for all $A, B \in$ $\mathcal{I}\left(A_{0}, \delta_{r}\right)$

$$
\begin{aligned}
\left\|\phi(A)^{-1} \cdot \phi(B)\right\| \leq & \left\|\left(d_{\mathbf{P}} f(\Phi(A))_{\mid \mathbb{H}}^{-1} \circ d_{\mathbf{P}} f(\Phi(A))_{\mid \mathbb{W}}\right)\left(A^{-1} B\right) \cdot \phi(A)^{-1} \cdot \phi(B)\right\| \\
& +\left\|\left(d_{\mathbf{P}} f(\Phi(A))_{\mid \mathbb{H}}^{-1} \circ d_{\mathbf{P}} f(\Phi(A))_{\mid \mathbb{W}}\right)\left(A^{-1} B\right)\right\| \\
\leq & \left.1 / 2\left(\left\|\phi(A)^{-1} \cdot \phi(B)\right\|+\| \phi(A)^{-1} A^{-1} B \phi(A)\right) \|\right)+C_{2}\left|\left(A^{-1} B\right)^{1}\right|_{\mathbb{R}^{m_{1}}} \\
\leq & \left.1 / 2\left\|\phi(A)^{-1} \cdot \phi(B)\right\|+\left(1 / 2+C_{2}\right) \| \phi(A)^{-1} A^{-1} B \phi(A)\right) \| .
\end{aligned}
$$

This inequality implies that (21) holds. As a consequence from (22) there is $C_{3}=C_{3}\left(A_{0}, r\right)>0$ such that for all $A, B \in \mathcal{I}\left(A_{0}, \delta_{r}\right)$

$$
\left\|\left(d_{\mathbf{P}} f(\Phi(A))_{\mid \mathbb{H}}^{-1} \circ d_{\mathbf{P}} f(\Phi(A))_{\mid \mathbb{W}}\right)\left(A^{-1} B\right) \cdot \phi(A)^{-1} \cdot \phi(B)\right\| \leq C_{3} \rho\left(\delta_{r}\right)\left\|\phi(A)^{-1} A^{-1} B \phi(A)\right\| .
$$

Now the proof that $\phi$ is u.i.d. at $A_{0}$ follows readily. Indeed, for all $A, B \in \mathcal{I}\left(A_{0}, \delta_{r}\right)$,

$$
\left\|\left(d_{\mathbf{P}} f\left(\Phi\left(A_{0}\right)\right)_{\mid \mathbb{H}}^{-1} \circ d_{\mathbf{P}} f\left(\Phi\left(A_{0}\right)\right)_{\mid \mathbb{W}}\right)\left(A^{-1} B\right) \cdot \phi(A)^{-1} \cdot \phi(B)\right\|
$$

(using again the fact $\|P\|=\left\|P^{-1}\right\|$ )

$$
\begin{aligned}
& \leq\left\|\left(d_{\mathbf{P}} f(\Phi(A))_{\mid \mathbb{H}}^{-1} \circ d_{\mathbf{P}} f(\Phi(A))_{\mid \mathbb{W}}\right)\left(A^{-1} B\right) \cdot \phi(A)^{-1} \cdot \phi(B)\right\| \\
& \quad+\left\|\left(d_{\mathbf{P}} f\left(\Phi\left(A_{0}\right)\right)_{\mid \mathbb{H}}^{-1} \circ d_{\mathbf{P}} f\left(\Phi\left(A_{0}\right)\right)_{\mid \mathbb{W}}\right)\left(A^{-1} B\right) \cdot\left(\left(d_{\mathbf{P}} f(\Phi(A))_{\mid \mathbb{H}}^{-1} \circ d_{\mathbf{P}} f(\Phi(A))_{\mid \mathbb{W}}\right)\left(A^{-1} B\right)\right)^{-1}\right\| \\
& \leq\left(C_{3} \rho\left(\delta_{r}\right)+o(1)\right)\left\|\phi(A)^{-1} A^{-1} B \phi(A)\right\|
\end{aligned}
$$

That is $\phi$ is uniformly intrinsic differentiable at $A_{0}$ and moreover

$$
d \phi_{A_{0}}=-d_{\mathbf{P}} f\left(\Phi\left(A_{0}\right)\right)_{\mid \mathbb{H}}^{-1} \circ d_{\mathbf{P}} f\left(\Phi\left(A_{0}\right)\right)_{\mid \mathbb{W}}
$$

This completes the proof of $(1) \Rightarrow(2)$.

$(2) \Rightarrow(1)$ The proof of this second part uses Whitney's Extension Theorem to prove the existence of an appropriate function $f \in \mathbb{C}_{\mathbb{G}}^{1}\left(\mathbb{G}, \mathbb{R}^{k}\right)$.

Let $\mathcal{F} \subset \mathcal{O}^{\prime}$ be a closed set such that $S \subset \mathcal{F}$. Let $f: \mathcal{F} \rightarrow \mathbb{R}^{k}$ and $g: \mathcal{F} \rightarrow \mathbf{M}_{k \times m_{1}}$ be given by

$$
f(Q):=0, \quad g(Q):=\left(\mathcal{I}_{k} \mid-\nabla^{\phi} \phi\left(\Phi^{-1}(Q)\right)\right)
$$

for all $Q \in \mathcal{F}$, where $\mathcal{I}_{k}$ is the $k \times k$ identity matrix and $\nabla^{\phi} \phi\left(\Phi^{-1}(Q)\right)$ is the unique $k \times\left(m_{1}-k\right)$ matrix associated to the intrinsic differential $d \phi_{\left(\Phi^{-1}(Q)\right)}$ of $\phi$ at $\Phi^{-1}(Q)$.

For any $\mathcal{K}$ compact in $\mathcal{F}, Q, Q^{\prime} \in \mathcal{K}$ and $\delta>0$ let

$$
\rho_{\mathcal{K}}(\delta):=\sup _{0<\left\|Q^{-1} Q^{\prime}\right\|<\delta} \frac{\left|g(Q)\left(Q^{-1} Q^{\prime}\right)^{1}\right|_{\mathbb{R}^{k}}}{\left\|Q^{-1} Q^{\prime}\right\|} .
$$


Whenever $Q=A \cdot \phi(A)$ and $Q^{\prime}=B \cdot \phi(B)$, because the homogeneous norm on the first layer is exactly the Euclidean norm we have

$$
\left|g(Q)\left(Q^{-1} Q^{\prime}\right)^{1}\right|_{\mathbb{R}^{k}}=\left\|\phi(B)-\phi(A)-\nabla^{\phi} \phi(A)\left(A^{-1} B\right)^{1}\right\|
$$

and, from (5) and the fact that $\mathbb{W}$ is a normal subgroup,

$$
c_{0}\left\|\phi(A)^{-1} A^{-1} B \phi(A)\right\|=c_{0}\left\|\mathbf{P}_{\mathbb{W}}\left(Q^{-1} \cdot Q^{\prime}\right)\right\| \leq\left\|Q^{-1} \cdot Q^{\prime}\right\| .
$$

Hence for any $A_{0} \in \Phi^{-1}(\mathcal{K})$

$$
\begin{aligned}
& \frac{\left|g(Q)\left(Q^{-1} Q^{\prime}\right)^{1}\right|_{\mathbb{R}^{k}}}{\left\|Q^{-1} Q^{\prime}\right\|} \\
& \leq \frac{1}{c_{0}} \frac{\left\|\phi(B)-\phi(A)-\nabla^{\phi} \phi(A)\left(A^{-1} B\right)^{1}\right\|}{\left\|\phi(A)^{-1} A^{-1} B \phi(A)\right\|} \\
& \leq \frac{1}{c_{0}}\left(\frac{\left\|\phi(B)-\phi(A)-\nabla^{\phi} \phi\left(A_{0}\right)\left(A^{-1} B\right)^{1}\right\|}{\left\|\phi(A)^{-1} A^{-1} B \phi(A)\right\|}+\frac{\left\|\left(\nabla^{\phi} \phi\left(A_{0}\right)-\nabla^{\phi} \phi(A)\right)\left(A^{-1} B\right)^{1}\right\|}{\left\|\phi(A)^{-1} A^{-1} B \phi(A)\right\|}\right) \\
& \leq \frac{1}{c_{0}}\left(\frac{\left\|\phi(B)-\phi(A)-\nabla^{\phi} \phi\left(A_{0}\right)\left(A^{-1} B\right)^{1}\right\|}{\left\|\phi(A)^{-1} A^{-1} B \phi(A)\right\|}+\left\|\nabla^{\phi} \phi\left(A_{0}\right)-\nabla^{\phi} \phi(A)\right\|\right)
\end{aligned}
$$

By the uniformly intrinsic differentiability of $\phi$ in $A_{0} \in \mathcal{O}^{\prime}$, for all $A, B \in \Phi^{-1}(\mathcal{K})$

$$
\lim _{\delta \rightarrow 0^{+}} \sup _{\left\|A_{0}^{-1} A\right\|<\delta} \sup _{0<\left\|A^{-1} B\right\|<\delta} \frac{\left\|\phi(B)-\phi(A)-\nabla^{\phi} \phi\left(A_{0}\right)\left(A^{-1} B\right)^{1}\right\|}{\left\|\phi(A)^{-1} A^{-1} B \phi(A)\right\|}=0
$$

and, consequently using also the continuity of the intrinsic differential $d \phi_{\left(\Phi^{-1}(Q)\right)}$ (see Proposition $3.7(3))$ we have

$$
\lim _{\delta \rightarrow 0^{+}} \rho_{\mathcal{K}}(\delta)=0 .
$$

Hence it is possible to apply Theorem 4.4 and we obtain the existence of $\hat{f} \in \mathbb{C}_{\mathbb{G}}^{1}\left(\mathbb{G}, \mathbb{R}^{k}\right)$ such that, for all $Q \in \mathcal{F}$

$$
\begin{gathered}
\hat{f}(Q)=f(Q)=0 \\
\nabla_{\mathbb{G}} \hat{f}(Q)=g(Q)=\left(\mathcal{I}_{k} \mid-\nabla^{\phi} \phi\left(\Phi^{-1}(Q)\right)\right)
\end{gathered}
$$

and, in particular, $\operatorname{rank} \nabla_{\mathbb{G}} \hat{f}(Q)=k$ for all $Q \in \mathcal{F}$.

Let $P=A \phi(A) \in S$ and $S^{\prime}:=\left\{Q \in \mathbb{G}: \hat{f}(Q)=0\right.$ and $\left.\operatorname{rank}\left(\nabla_{\mathbb{G}} \hat{f}(Q)\right)=k\right\}$. We just prove that there is a neighbourhood $\mathcal{U}$ of $P$ such that $\mathcal{U} \cap S \subset S^{\prime}$ and $\operatorname{rank} \nabla_{\mathbb{G}} \hat{f}(Q)=k$ for all $Q \in S$.

In order to get the implication $(2) \Rightarrow(1)$ we need to find a certain neighbourhood $\mathcal{U}\left(P, r_{0}\right)$ of $P$ such that

$$
\mathcal{U}\left(P, r_{0}\right) \cap S=\mathcal{U}\left(P, r_{0}\right) \cap S^{\prime}
$$

As $P \in \mathcal{U} \cap S \subset S^{\prime}$ we get

$$
\hat{f}(P)=0, \quad \nabla_{\mathbb{G}} \hat{f}(P)=\left(\mathcal{I}_{k} \mid-\nabla^{\phi} \phi\left(\Phi^{-1}(P)\right)\right)
$$

and by Implicit Function Theorem there exist an open neighborhood $\mathcal{U}^{\prime}$ of $P$ and a continuous function $\phi^{\prime}: \mathcal{I}\left(A, \delta^{\prime}\right) \rightarrow \mathbb{H}$ such that

$$
\Phi^{\prime}: \mathcal{I}\left(A, \delta^{\prime}\right) \rightarrow S^{\prime} \cap \overline{\mathcal{U}^{\prime}}
$$

is an homeomorphism given by

$$
\Phi^{\prime}(B)=B \phi^{\prime}(B), \quad \text { for all } B \in \mathcal{I}\left(A, \delta^{\prime}\right) .
$$

Moreover $\Phi^{\prime-1}\left(S^{\prime} \cap \overline{\mathcal{U}^{\prime}}\right)$ is an open subset of $\mathcal{I}\left(A, \delta^{\prime}\right)$, with $A \in \Phi^{\prime-1}\left(S^{\prime} \cap \overline{\mathcal{U}^{\prime}}\right)$ because $P \in$ $S^{\prime} \cap \mathcal{U}^{\prime}$. So there is $\delta^{\prime \prime} \in\left(0, \delta^{\prime}\right)$ such that $\mathcal{I}\left(A, \delta^{\prime \prime}\right) \subset \Phi^{\prime-1}\left(S^{\prime} \cap \mathcal{U}^{\prime}\right)$ and, by the uniqueness of the parametrization, we obtain that $\Phi^{\prime} \equiv \Phi$ on $\mathcal{I}\left(A, \delta^{\prime \prime}\right)$. 
Now, set $\mathcal{U}^{\prime \prime}$ and $\mathcal{U}^{\prime \prime \prime}$ be an open neighborhood of $P$ in $\mathbb{G}$ such that

$$
S \cap \mathcal{U}^{\prime \prime}=\Phi\left(\mathcal{I}\left(A, \delta^{\prime \prime}\right)\right)=\Phi^{\prime}\left(\mathcal{I}\left(A, \delta^{\prime \prime}\right)\right)=S^{\prime} \cap \mathcal{U}^{\prime \prime \prime}
$$

and set $r_{0}>0$ be such that $\mathcal{U}\left(P, r_{0}\right) \subset \mathcal{U}^{\prime \prime} \cap \mathcal{U}^{\prime \prime \prime}$. Therefore, since the last equality holds, we observe that $\mathcal{U}\left(P, r_{0}\right) \cap S=\mathcal{U}\left(P, r_{0}\right) \cap S^{\prime}$, i.e. (23) holds.

This completes the proof of the theorem. fact:

We notice that the proof of $(1) \Rightarrow(2)$ and Implicit Function Theorem give the following general

Theorem 4.6. Let $S$ be a $k$-codimensional $\mathbb{G}$-regular surface and $\mathcal{U}$ be a neighbourhood of $P \in S$. We have, for $f \in \mathbb{C}_{\mathbb{G}}^{1}\left(\mathcal{U} ; \mathbb{R}^{k}\right)$, that

$$
S \cap \mathcal{U}=\{Q \in \mathcal{U}: f(Q)=0\},
$$

and $\mathbb{W}=\operatorname{ker}\left(d_{\mathbf{P}} f(P)\right)$ has a complementary horizontal $k$-dimensional subgroup $\mathbb{H}$ in $\mathbb{G}$. Then there exist $\mathcal{O}$ open in $\mathbb{W}, \phi: \mathcal{O} \rightarrow \mathbb{H}$ u.i.d. and a smaller neighbourhood of $P, \mathcal{U}^{\prime} \subset \mathcal{U}$, such that

$$
S \cap \mathcal{U}^{\prime}=\operatorname{graph}(\phi) \cap \mathcal{U}^{\prime} .
$$

Proof. The existence of $\phi: \mathcal{O} \subset \mathbb{W} \rightarrow \mathbb{V}$ follows from Implicit Function Theorem. Moreover $\phi$ is uniformly intrinsic differentiable in $\mathcal{O}$ by Theorem 4.1. By the definition of $\mathbb{W}$ and $\mathbb{H}$ we have that $d_{\mathbf{P}} f(P): \mathbb{H} \rightarrow \mathbb{R}^{k}$ is bijective and by continuity this holds also in some neighbourhood of $P$. The same holds for the map $Q \mapsto\left(d_{\mathbf{P}} f(Q)_{\mathbb{H}}\right)^{-1}$ because the map $Q \mapsto d_{\mathbf{P}} f(Q)_{\mid \mathbb{H}}$ is a linear isomorphism between $\mathbb{H}$ and $\mathbb{R}^{k}$ which depends continuously on $Q$ in a proper neighbourhood of $P$. Then we can argue as in $(1) \Rightarrow(2)$ of Theorem 4.1 and we obtain the thesis.

As a corollary of Theorem 4.1 and Theorem 2.2, we get a comparison between the Reifenberg vanishing flat set and the uniformly intrinsic differentiable map:

Corollary 4.7. Let $\mathbb{W}$ and $\mathbb{H}$ be complementary subgroups of a Carnot group $\mathbb{G}$ with $\mathbb{H}$ horizontal and $k$ dimensional. Let $\mathcal{O}$ be open in $\mathbb{W}, \phi: \mathcal{O} \subset \mathbb{W} \rightarrow \mathbb{H}$ and $S:=$ graph $(\phi)$. Moreover we assume that there is a family $\left\{\mathbb{W}_{P}: P \in S\right\}$ of vertical subgroup complementary to $\mathbb{H}$ (hence of codimension $k$ ) and for every relatively compact subset $S^{\prime} \Subset S$ there is an increasing function $\beta:(0, \infty) \rightarrow(0, \infty), \beta(t) \rightarrow 0^{+}$when $t \rightarrow 0^{+}$, such that

$$
\operatorname{dist}_{d}\left(\mathcal{U}(P, r) \cap S, \mathcal{U}(P, r) \cap\left(P \cdot \mathbb{W}_{P}\right)\right) \leq \beta(r) r, \quad r>0
$$

for any $P \in S^{\prime}$. Then, for every $A_{0} \in \mathcal{O}$,

(1) there are a neighbourhood $\mathcal{U}$ of $A_{0} \cdot \phi\left(A_{0}\right)$ and $f \in \mathbb{C}_{\mathbb{G}}^{1}\left(\mathcal{U} ; \mathbb{R}^{k}\right)$ such that

$$
\begin{aligned}
& S \cap \mathcal{U}=\{P \in \mathcal{U}: f(P)=0\} \\
& d_{\mathbf{P}} f(Q)_{\mid \mathbb{H}}: \mathbb{H} \rightarrow \mathbb{R}^{k} \quad \text { is bijective for all } Q \in \mathcal{U}
\end{aligned}
$$

and $Q \mapsto\left(d_{\mathbf{P}} f(Q)_{\mathbb{H}}\right)^{-1}$ is continuous.

(2) $\phi$ is u.i.d. in a neighbourhood $\mathcal{O}^{\prime} \subset \mathcal{O}$ of $A_{0}$.

Proof. (1) Theorem 2.2 states there are a neighbourhood $\mathcal{U}$ of $A_{0} \cdot \phi\left(A_{0}\right)$ and $f \in \mathbb{C}_{\mathbb{G}}^{1}\left(\mathcal{U} ; \mathbb{R}^{k}\right)$ such that $S \cap \mathcal{U}=\{P \in \mathcal{U}: f(P)=0\}$. Using again Theorem 2.2 we know that $\mathbb{W}_{P}=\operatorname{ker}\left(d_{\mathbf{P}} f(P)\right)$ and so the thesis follows from the fact that $\mathbb{W}_{P}$ is complementary subgroup of $\mathbb{H}$.

The condition (2) is equivalent to the condition (1) thanks to Theorem 4.1.

Corollary 4.8. Under the same assumptions of Theorem 4.1, if $S:=\operatorname{graph}(\phi)$ satisfies the condition (1) of Theorem 4.1, then

(1) the function $B \mapsto d \phi(B)$ is continuous in $\mathcal{O}$. 
(2) $\phi \in h_{\text {loc }}^{1 / \kappa}(\mathcal{O})$, that is $\phi \in \mathbb{C}(\mathcal{O}, \mathbb{R})$ and for all $\mathcal{F} \Subset \mathcal{O}$ and $A, B \in \mathcal{F}$

$$
\lim _{r \rightarrow 0^{+}} \sup _{0<\left\|A^{-1} B\right\|<r} \frac{\|\phi(B)-\phi(A)\|}{\left\|A^{-1} B\right\|^{1 / \kappa}}=0 .
$$

Observe that u.i.d. functions do exist. In particular, when $\mathbb{H}$ is a horizontal subgroup, $\mathbb{H}$ valued Euclidean $\mathbb{C}^{1}$ functions are u.i.d.

Theorem 4.9. If $\mathbb{W}$ and $\mathbb{H}$ are complementary subgroups of a Carnot group $\mathbb{G}$ with $\mathbb{H}$ horizontal and $k$ dimensional. If $\mathcal{O}$ is open in $\mathbb{W}$ and $\phi: \mathcal{O} \subset \mathbb{W} \rightarrow \mathbb{H}$ is such that $\phi \in \mathbb{C}^{1}(\mathcal{O}, \mathbb{H})$ then $\phi$ is u.i.d. in $\mathcal{O}$.

Proof. Assume without loss of generality that $X_{1}, \ldots, X_{k}$ are horizontal vector fields such that $\mathbb{H}=\exp \left(\operatorname{span}\left\{X_{1}, \ldots, X_{k}\right\}\right)$. Then $\phi=\exp \sum_{i=1}^{k} \phi_{i} X_{i}$, where $\phi_{i}: \mathcal{O} \rightarrow \mathbb{R}$ are $\mathbb{C}^{1}$ functions. Let $\mathcal{U}:=\mathcal{O} \cdot \mathbb{H}$. If $P \in \mathcal{U}$ then $P=P_{\mathbb{W}} \cdot \exp \sum_{i=1}^{k} x_{i} X_{i}$ with $P_{\mathbb{W}} \in \mathcal{O}$ and we define $f: \mathcal{U} \rightarrow \mathbb{R}^{k}$ as

$$
f(P)=\left(x_{1}-\phi_{1}\left(P_{\mathbb{W}}\right), \ldots, x_{k}-\phi_{k}\left(P_{\mathbb{W}}\right)\right) .
$$

With this definition $f \in \mathbb{C}^{1}\left(\mathcal{U}, \mathbb{R}^{k}\right)$, hence $f \in \mathbb{C}_{\mathbb{G}}^{1}\left(\mathcal{U}, \mathbb{R}^{k}\right)$. Moreover

$$
X_{l} f_{j}(P)=\frac{d}{d s} f_{j}\left(P \exp \left(s X_{l}\right)\right)_{\mid s=0}= \begin{cases}1 & \text { if } j=l \\ 0 & \text { if } j \neq l\end{cases}
$$

hence $\operatorname{rank}\left(\nabla_{\mathbb{G}} f(P)\right)=k$.

By construction $\{Q \in \mathcal{U}: f(Q)=0\}=\operatorname{graph}(\phi)$ then graph $(\phi)$ is a non critical level set of $f \in \mathbb{C}_{\mathbb{G}}^{1}\left(\mathcal{U}, \mathbb{R}^{k}\right)$ hence is a $\mathbb{G}$-regular surface. From Theorem 4.1 it follows that $\phi$ is u.i.d. in $\mathcal{O}$.

\section{1-Codimensional INTRINSIC GRAPHS IN CARNOT GROUPS OF STEP 2}

In this section we characterize uniformly intrinsic differentiable functions $\phi: \mathcal{O} \subset \mathbb{W} \rightarrow \mathbb{V}$, when $\mathbb{V}$ is one dimensional and horizontal, in terms of existence and continuity of suitable intrinsic derivatives of $\phi$. Intrinsic derivatives are first order non linear differential operators depending on the structure of the ambient space $\mathbb{G}$ and on the two complementary subgroups $\mathbb{W}$ and $\mathbb{V}$.

In order to do this we have to restrict the ambient space $\mathbb{G}$ under consideration to a subclass of Carnot groups of step two. These groups, denoted here as groups of class $\mathcal{B}$, are described in the next subsection where we follow the notations of Chapter 3 of [6].

\subsection{Carnot groups of class $\mathcal{B}$.}

Definition 5.1. We say that $\mathbb{G}:=\left(\mathbb{R}^{m+n}, \cdot, \delta_{\lambda}\right)$ is a Carnot group of class $\mathcal{B}$ if there are $n$ linearly independent, skew-symmetric $m \times m$ real matrices $\mathcal{B}^{(1)}, \ldots, \mathcal{B}^{(n)}$ such that for all $P=\left(P^{1}, P^{2}\right)$ and $Q=\left(Q^{1}, Q^{2}\right) \in \mathbb{R}^{m} \times \mathbb{R}^{n}$ and for all $\lambda>0$

$$
P \cdot Q=\left(P^{1}+Q^{1}, P^{2}+Q^{2}+\frac{1}{2}\left\langle\mathcal{B} P^{1}, Q^{1}\right\rangle\right)
$$

where $\left\langle\mathcal{B} P^{1}, Q^{1}\right\rangle:=\left(\left\langle\mathcal{B}^{(1)} P^{1}, Q^{1}\right\rangle, \ldots,\left\langle\mathcal{B}^{(n)} P^{1}, Q^{1}\right\rangle\right)$ and $\langle\cdot, \cdot\rangle$ is the inner product in $\mathbb{R}^{m}$ and

$$
\delta_{\lambda} P:=\left(\lambda P^{1}, \lambda^{2} P^{2}\right) \text {. }
$$

Under these assumptions $\mathbb{G}$ is a Carnot group of step 2 with $\mathbb{R}^{m}$ the horizontal layer and $\mathbb{R}^{n}$ the vertical layer.

We recall also that, by Proposition 3.4. [3], for any homogeneous norm in $\mathbb{G}$ there is $c_{1}>1$ such that for all $P=\left(P^{1}, P^{2}\right) \in \mathbb{G}$

$$
c_{1}^{-1}\left(\left|P^{1}\right|_{\mathbb{R}^{m}}+\left|P^{2}\right|_{\mathbb{R}^{n}}^{1 / 2}\right) \leq\|P\| \leq c_{1}\left(\left|P^{1}\right|_{\mathbb{R}^{m}}+\left|P^{2}\right|_{\mathbb{R}^{n}}^{1 / 2}\right)
$$


From now on we will depart slightly from the notations of the previous sections. Precisely, instead of writing $P=\left(p_{1}, \ldots, p_{m+n}\right)$ we will write

$$
P=\left(x_{1}, \ldots, x_{m}, y_{1}, \ldots, y_{n}\right) .
$$

With this notation, when $\mathcal{B}^{(s)}:=\left(b_{i j}^{s}\right)_{i, j=1}^{m}$, a basis of the Lie algebra $\mathfrak{g}$ of $\mathbb{G}$, is given by the $m+n$ left invariant vector fields

$$
X_{j}(P)=\partial_{x_{j}}+\frac{1}{2} \sum_{s=1}^{n} \sum_{i=1}^{m} b_{j i}^{s} x_{i} \partial_{y_{s}}, \quad Y_{s}(P)=\partial_{y_{s}},
$$

where $j=1, \ldots, m$, and $s=1, \ldots, n$.

Remark 5.1. The space of skew-symmetric $m \times m$ matrices has dimension $\frac{m(m-1)}{2}$. Hence in any group $\mathbb{G}$ of class $\mathcal{B}$ the dimensions $m$ of the horizontal layer and $n$ of the vertical layer are related by the inequality

$$
n \leq \frac{m(m-1)}{2} .
$$

Remark 5.2. Heisenberg groups are groups of class $\mathcal{B}$. Indeed $\mathbb{H}^{k}=\mathbb{R}^{2 k} \times \mathbb{R}$ and the group law is of the form (24) with

$$
\mathcal{B}^{(1)}=\left(\begin{array}{cc}
0 & \mathcal{I}_{k} \\
-\mathcal{I}_{k} & 0
\end{array}\right)
$$

where $\mathcal{I}_{k}$ is the $k \times k$ identity matrix.

More generally, $\mathrm{H}$-type groups are examples of groups of class $\mathcal{B}$ (see Definition 3.6.1 and Remark 3.6.7 in [6]). In this case $\mathbb{G}=\mathbb{R}^{m} \times \mathbb{R}^{n}$ with

$$
n<8 p+q, \quad \text { with } m=\text { (odd) } 2^{4 p+q} \text { and } 0 \leq q \leq 3 .
$$

Observe that if $m$ is odd then $n=0$, hence in the non trivial cases $m$ is even. The composition law is of the form (24) where the matrices $\mathcal{B}^{(1)}, \ldots, \mathcal{B}^{(n)}$ have the following additional properties:

(1) $\mathcal{B}^{(s)}$ is an $m \times m$ orthogonal matrix for all $s=1, \ldots n$

$(2) \mathcal{B}^{(s)} \mathcal{B}^{(l)}=-\mathcal{B}^{(l)} \mathcal{B}^{(s)}$ for every $s, l=1, \ldots, n$ with $s \neq l$.

Another example of Carnot groups of class $\mathcal{B}$ is provided by the class $\mathbb{F}_{m, 2}$ of free groups of step-2 (see Section 3.3 in [6]). Here $\mathbb{F}_{m, 2}=\mathbb{R}^{m} \times \mathbb{R}^{\frac{m(m-1)}{2}}$ and the composition law (24) is defined by the matrices $\mathcal{B}^{(s)} \equiv \mathcal{B}^{(i, j)}$ where $1 \leq j<i \leq m$ and $\mathcal{B}^{(i, j)}$ has entries -1 in position $(i, j), 1$ in position $(j, i)$ and 0 everywhere else.

Notice that Heisenberg groups are H-type groups while $\mathbb{H}^{1}$ is also a free step- 2 group.

5.2. The intrinsic gradient. Let $\mathbb{G}=\left(\mathbb{R}^{m+n}, \cdot, \delta_{\lambda}\right)$ be a group of class $\mathcal{B}$ as in Definition 5.1 and $\mathbb{W}, \mathbb{V}$ be complementary subgroups in $\mathbb{G}$ with $\mathbb{V}$ horizontal and one dimensional.

Remark 5.3. To keep notations simpler, through all this section we assume, without loss of generality, that the complementary subgroups $\mathbb{W}, \mathbb{V}$ are

$$
\mathbb{V}:=\left\{\left(x_{1}, 0 \ldots, 0\right)\right\}, \quad \mathbb{W}:=\left\{\left(0, x_{2}, \ldots, x_{m+n}\right)\right\} .
$$

This amounts simply to a linear change of variables in the first layer of the algebra $\mathfrak{g}$. If we denote $\mathcal{M}$ a non singular $m \times m$ matrix, the linear change of coordinates associated to $\mathcal{M}$ is

$$
P=\left(P^{1}, P^{2}\right) \mapsto\left(\mathcal{M} P^{1}, P^{2}\right)
$$

The new composition law $\star$ in $\mathbb{R}^{m+n}$, obtained by writing $\cdot$ in the new coordinates, is

$$
\left(\mathcal{M} P^{1}, P^{2}\right) \star\left(\mathcal{M} Q^{1}, Q^{2}\right):=\left(\mathcal{M} P^{1}+\mathcal{M} Q^{1}, P^{2}+Q^{2}+\frac{1}{2}\left\langle\tilde{\mathcal{B}} \mathcal{M} P^{1}, \mathcal{M} Q^{1}\right\rangle\right),
$$


where $\tilde{\mathcal{B}}:=\left(\tilde{\mathcal{B}}^{(1)}, \ldots, \tilde{\mathcal{B}}^{(n)}\right)$ and $\tilde{\mathcal{B}}^{(s)}=\left(\mathcal{M}^{-1}\right)^{T} \mathcal{B}^{(s)} \mathcal{M}^{-1}$ for $s=1, \ldots, n$. It is easy to check that the matrices $\tilde{\mathcal{B}}^{(1)}, \ldots, \tilde{\mathcal{B}}^{(n)}$ are skew-symmetric and that $\left(\mathbb{R}^{m+n}, \star, \delta_{\lambda}\right)$ is a Carnot group of class $\mathcal{B}$ isomorphic to $\mathbb{G}=\left(\mathbb{R}^{m+n}, \cdot, \delta_{\lambda}\right)$.

When $\mathbb{V}$ and $\mathbb{W}$ are defined as in (26) there is a natural inclusion $i: \mathbb{R}^{m+n-1} \rightarrow \mathbb{W}$ such that, for all $\left(x_{2}, \ldots x_{m}, y_{1}, \ldots, y_{n}\right) \in \mathbb{R}^{m+n-1}$,

$$
i\left(\left(x_{2}, \ldots x_{m}, y_{1}, \ldots, y_{n}\right)\right):=\left(0, x_{2}, \ldots x_{m}, y_{1}, \ldots, y_{n}\right) \in \mathbb{W}
$$

If $\mathcal{O}$ and $\phi$ are respectively an open set in $\mathbb{R}^{m+n-1}$ and a function $\phi: \mathcal{O} \rightarrow \mathbb{R}$ we denote $\hat{\mathcal{O}}:=$ $i(\mathcal{O}) \subset \mathbb{W}$ and $\hat{\phi}: \hat{\mathcal{O}} \rightarrow \mathbb{V}$ the function defined as

$$
\hat{\phi}(i(A)):=(\phi(A), 0, \ldots, 0)
$$

for all $A \in \mathcal{O}$.

From (19) in Theorem 4.1, if $\hat{\phi}: \hat{\mathcal{O}} \subset \mathbb{W} \rightarrow \mathbb{V}$ is such that graph $(\hat{\phi})$ is locally a non critical level set of $f \in \mathbb{C}_{\mathbb{G}}^{1}(\mathbb{G}, \mathbb{R})$ with $X_{1} f \neq 0$, then $\hat{\phi}$ is u.i.d. in $\hat{\mathcal{O}}$ and the following representation of the intrinsic gradient $\nabla^{\hat{\phi}} \hat{\phi}$ holds

$$
\nabla^{\hat{\phi}} \hat{\phi}(P)=-\left(\frac{X_{2} f}{X_{1} f}, \ldots, \frac{X_{m} f}{X_{1} f}\right)(P \cdot \hat{\phi}(P))
$$

for all $P \in \hat{\mathcal{O}}$.

Moreover, there is an explicit expression of the intrinsic gradient of $\hat{\phi}$, not involving $f$, but only derivatives of the real valued function $\phi$. In Proposition 5.4 (see Proposition 4.6 in [2] in Heisenberg groups) we motivate the following definition:

Definition 5.2. Let $\mathcal{O}$ be open in $\mathbb{R}^{m+n-1}, \psi: \mathcal{O} \rightarrow \mathbb{R}$ be continuous in $\mathcal{O}$. The intrinsic derivatives $D_{j}^{\psi}$, for $j=2, \ldots, m$, are the differential operators with continuous coefficients

$$
\begin{aligned}
D_{j}^{\psi} & :=\partial_{x_{j}}+\sum_{s=1}^{n}\left(\psi b_{j 1}^{s}+\frac{1}{2} \sum_{l=2}^{m} x_{l} b_{j l}^{s}\right) \partial_{y_{s}} \\
& =X_{j \mid \mathbb{W}}+\psi \sum_{s=1}^{n} b_{j 1}^{s} Y_{s \mid \mathbb{W}}
\end{aligned}
$$

where, in the second line with abuse of notation, we denote with the same symbols $X_{j}$ and $Y_{s}$ the vector fields acting on functions defined in $\mathcal{O}$.

If $\hat{\psi}:=(\psi, 0, \ldots, 0): \hat{\mathcal{O}} \rightarrow \mathbb{V}$, we denote intrinsic horizontal gradient $\nabla^{\hat{\psi}}$ the differential operator

$$
\nabla^{\hat{\psi}}:=\left(D_{2}^{\psi}, \ldots, D_{m}^{\psi}\right) \text {. }
$$

Proposition 5.4. Let $\mathbb{G}:=\left(\mathbb{R}^{m+n}, \cdot, \delta_{\lambda}\right)$ be a Carnot group of class $\mathcal{B}$ and $\mathbb{V}, \mathbb{W}$ the complementary subgroups defined in (26). Let $\mathcal{U}$ be open in $\mathbb{G}, f \in \mathbb{C}_{\mathbb{G}}^{1}(\mathcal{U}, \mathbb{R})$ with $X_{1} f>0$ and assume that $S:=\{P \in \mathcal{U}: f(P)=0\}$ is non empty. Then

(i) for every $P \in \mathcal{U}$ there exist $\mathcal{U}^{\prime}$ open neighbourhood of $P$ in $\mathbb{G}, \hat{\mathcal{O}}$ open in $\mathbb{W}$ and $\hat{\psi}: \hat{\mathcal{O}} \rightarrow \mathbb{V}$ such that $S \cap \mathcal{U}^{\prime}=\operatorname{graph}(\hat{\psi}) \cap \mathcal{U}^{\prime}$. Moreover $\hat{\psi}$ is u.i.d. in $\hat{\mathcal{O}}$ and the distributional intrinsic gradient $\left(D_{2}^{\psi} \psi, \ldots, D_{m}^{\psi} \psi\right)$ of the associated function $\psi: \mathcal{O} \rightarrow \mathbb{R}$ is defined as

$$
D_{j}^{\psi} \psi=X_{j} \psi+\psi \sum_{s=1}^{n} b_{j 1}^{s} Y_{s} \psi, \quad \text { for } j=2, \ldots, m .
$$

Moreover the distributional intrinsic gradient of $\psi$ has a continuous representative, which is $\nabla^{\hat{\psi}} \hat{\psi}$ (continuous because of (28) ), i.e.

$$
\nabla^{\hat{\psi}} \hat{\psi}(i(\cdot))=\left(\begin{array}{c}
\left.D_{2}^{\psi} \psi(\cdot), \ldots, D_{m}^{\psi} \psi(\cdot)\right) \\
22
\end{array}\right.
$$


holds in the sense of distribution.

(ii) The subgraph $\mathcal{E}:=\{P \in \mathcal{U}: f(P)<0\}$ has locally finite $\mathbb{G}$-perimeter in $\mathcal{U}$ and its $\mathbb{G}$ perimeter measure $|\partial \mathcal{E}|_{\mathbb{G}}$ has the integral representation

$$
|\partial \mathcal{E}|_{\mathbb{G}}(\mathcal{F})=\int_{\Phi^{-1}(\mathcal{F})} \sqrt{1+\left|\nabla^{\hat{\psi}} \hat{\psi}\right|_{\mathbb{R}^{m-1}}^{2}} d \mathcal{L}^{m+n-1}
$$

for every Borel set $\mathcal{F} \subset \mathcal{U}$ where $\Phi: \mathcal{O} \rightarrow \mathbb{G}$ is defined as $\Phi(A):=i(A) \cdot \hat{\psi}(i(A))$ for all $A \in \mathcal{O}$.

Proof. The first part of statement (i) follows from Implicit Function Theorem and Theorem 4.6, Moreover $\hat{\psi}$ is uniformly intrinsic differentiable in $\hat{\mathcal{O}}$ by Theorem 4.1. Let $\psi: \mathcal{O} \subset \mathbb{R}^{m+n-1} \rightarrow \mathbb{R}$ be the real valued continuous function associated to $\hat{\psi}$ as in (27).

Fix $A \in \mathcal{O}$. Let $P:=\Phi(A)$ where $\Phi$ is the graph map of $\hat{\psi}$ defined as $\Phi(A):=i(A) \cdot \hat{\psi}(i(A))$. Because $S:=\operatorname{graph}(\hat{\psi})$ is a $\mathbb{G}$-regular hypersurface we know that there are $r>0, \delta>0$ and $f \in \mathbb{C}_{\mathbb{G}}^{1}(\mathcal{U}(P, r), \mathbb{R})$ such that $f \circ \Phi=0$ in $\mathcal{I}(A, \delta)$.

Now we use some results proved in Implicit Function Theorem in [13] (see Theorem 2.1 in [13]). Arguing as in Step 1 of this theorem we can prove the existence of $0<r^{\prime}<r$ and of a family $\left(f_{\epsilon}\right)_{\epsilon>0} \subset \mathbb{C}^{1}\left(\mathcal{U}\left(P, r^{\prime}\right), \mathbb{R}\right)$ such that

$$
f_{\epsilon} \rightarrow f \text { and } \nabla_{\mathbb{G}} f_{\epsilon} \rightarrow \nabla_{\mathbb{G}} f \quad \text { uniformly on } \mathcal{U}\left(P, r^{\prime}\right) \text {, as } \epsilon \rightarrow 0 .
$$

Moreover as in Step 3 of Implicit Function Theorem in [13] there is $\left(\psi_{\epsilon}\right)_{\epsilon>0} \subset \mathbb{C}^{1}(\mathcal{I}(A, \delta), \mathbb{R})$ satisfying

$$
\psi_{\epsilon} \rightarrow \psi \text { and }-\frac{\widehat{\nabla_{\mathbb{G}}} f_{\epsilon}}{X_{1} f_{\epsilon}} \circ \Phi_{\epsilon} \rightarrow-\frac{\widehat{\nabla_{\mathbb{G}}} f}{X_{1} f} \circ \Phi
$$

uniformly on $\mathcal{I}(A, \delta)$ as $\epsilon \rightarrow 0$, where $\widehat{\nabla_{\mathbb{G}}} f:=\left(X_{2} f, \ldots, X_{m} f\right)$ and the graph maps $\Phi_{\epsilon}$ of $\hat{\psi}_{\epsilon}=$ $\left(\psi_{\epsilon}, 0, \ldots, 0\right)$ are such that $f_{\epsilon} \circ \Phi_{\epsilon} \equiv 0$.

Moreover, the set $S_{\epsilon}:=\left\{Q \in \mathcal{U}\left(P, r^{\prime}\right): f_{\epsilon}(Q)=0\right\} \supset \Phi_{\epsilon}(\mathcal{I}(A, \delta))$ is an Euclidean $\mathbb{C}^{1}$ surface. Hence, because $f_{\epsilon} \in \mathbb{C}^{1}\left(\mathcal{U}\left(P, r^{\prime}\right)\right) \subset \mathbb{C}_{\mathbb{G}}^{1}\left(\mathcal{U}\left(P, r^{\prime}\right)\right)$ and because of Theorem 4.1 we have that $\hat{\psi}_{\epsilon}$ (i.e. the parametrization of $S_{\epsilon}$ ) is uniformly intrinsic differentiable and by (28), (30) and (31) we get

$$
\nabla^{\hat{\psi}_{\epsilon}} \hat{\psi}_{\epsilon}(i(\cdot))=-\frac{\widehat{\nabla_{\mathbb{G}}} f_{\epsilon}}{X_{1} f_{\epsilon}} \circ \Phi_{\epsilon}(\cdot) \rightarrow \nabla^{\hat{\psi}} \hat{\psi}(i(\cdot))
$$

uniformly on $\mathcal{I}(A, \delta)$ as $\epsilon \rightarrow 0$.

Differentiating the equality $f_{\epsilon}\left(i(B) \cdot \hat{\psi}_{\epsilon}(i(B))\right)=f_{\epsilon}\left(\Phi_{\epsilon}(B)\right)=0$, for $B=\left(x_{2}, \ldots, x_{m}, y_{1}, \ldots, y_{n}\right) \epsilon$ $\mathcal{I}(A, \delta)$, we get

$$
\begin{array}{ll}
\partial_{x_{j}} \psi_{\epsilon}(B)=-\frac{\partial_{x_{j}} f_{\epsilon}\left(\Phi_{\epsilon}(B)\right)-\frac{1}{2} \psi_{\epsilon}(B) \sum_{s=1}^{n} b_{j 1}^{s} \partial_{y_{s}} f_{\epsilon}\left(\Phi_{\epsilon}(B)\right)}{X_{1} f_{\epsilon}\left(\Phi_{\epsilon}(B)\right)}, & j=2, \ldots, m \\
\partial_{y_{s}} \psi_{\epsilon}(B)=-\frac{\partial_{y_{s}} f_{\epsilon}\left(\Phi_{\epsilon}(B)\right)}{X_{1} f_{\epsilon}\left(\Phi_{\epsilon}(B)\right)}, & s=1, \ldots, n
\end{array}
$$

Then 


$$
\begin{aligned}
& -\frac{X_{j} f_{\epsilon}\left(\Phi_{\epsilon}(B)\right)}{X_{1} f_{\epsilon}\left(\Phi_{\epsilon}(B)\right)} \\
& =-\frac{\partial_{x_{j}} f_{\epsilon}\left(\Phi_{\epsilon}(B)\right)+\frac{1}{2} \sum_{s=1}^{n}\left(\psi_{\epsilon}(B) b_{j 1}^{s}+\sum_{l=2}^{m} x_{l} b_{j l}^{s}\right) \partial_{y_{s}} f_{\epsilon}\left(\Phi_{\epsilon}(B)\right)}{X_{1} f_{\epsilon}\left(\Phi_{\epsilon}(B)\right)}
\end{aligned}
$$

(here we use the skew symmetry of the matrices $\mathcal{B}^{(s)}$ )

$$
\begin{aligned}
&=-\frac{\partial_{x_{j}} f_{\epsilon}\left(\Phi_{\epsilon}(B)\right)-\frac{1}{2} \psi_{\epsilon}(B) \sum_{s=1}^{n} b_{j 1}^{s} \partial_{y_{s}} f_{\epsilon}\left(\Phi_{\epsilon}(B)\right)}{X_{1} f_{\epsilon}\left(\Phi_{\epsilon}(B)\right)} \\
&-\frac{\sum_{s=1}^{n}\left(\psi_{\epsilon}(B) b_{j 1}^{s}+\frac{1}{2} \sum_{l=2}^{m} x_{l} b_{j l}^{s}\right) \partial_{y_{s}} f_{\epsilon}\left(\Phi_{\epsilon}(B)\right)}{X_{1} f_{\epsilon}\left(\Phi_{\epsilon}(B)\right)} \\
&=\partial_{x_{j}} \psi_{\epsilon}(B)+\sum_{s=1}^{n}\left(\psi_{\epsilon}(B) b_{j 1}^{s}+\frac{1}{2} \sum_{l=2}^{m} x_{l} b_{j l}^{s}\right) \partial_{y_{s}} \psi_{\epsilon}(B) .
\end{aligned}
$$

Then, from (28)

$$
D_{j}^{\psi_{\epsilon}} \psi_{\epsilon}(B)=\partial_{x_{j}} \psi_{\epsilon}(B)+\sum_{s=1}^{n}\left(\psi_{\epsilon}(B) b_{j 1}^{s}+\frac{1}{2} \sum_{l=2}^{m} x_{l} b_{j l}^{s}\right) \partial_{y_{s}} \psi_{\epsilon}(B) .
$$

Letting $\epsilon \rightarrow 0^{+}$(29) follows from (31) and so

$$
\nabla^{\hat{\psi}} \hat{\psi}(i(\cdot))=\left(D_{2}^{\psi} \psi(\cdot), \ldots, D_{m}^{\psi} \psi(\cdot)\right)
$$

holds in the sense of distribution.

Finally using again Implicit Function Theorem in [13], we know that

$$
|\partial \mathcal{E}|_{\mathbb{G}}(\mathcal{F})=\int_{\Phi^{-1}(\mathcal{F})} \frac{\left|\nabla_{\mathbb{G}} f(\Phi(B))\right|_{\mathbb{R}^{m+n-1}}}{X_{1} f(\Phi(B))} d \mathcal{L}^{m+n-1}(B)
$$

and, consequently, the integral representation of the perimeter $|\partial \mathcal{E}|_{\mathbb{G}}$ is true because, from (28)), $D_{j}^{\psi} \psi=-\frac{X_{j} f}{X_{1} f} \circ \Phi$, in the sense of distributions, for $j=2, \ldots, m$. This completes the proof of (ii).

Remark 5.5. We emphasize that the intrinsic horizontal gradient $\nabla^{\hat{\psi}}$ defined after Definition 5.2 and the continuous representative of the distributional intrinsic gradient as in (19) are different; but according to the approximation argument of Proposition 5.4, they are equal in distributional sense.

In the following proposition we prove that, if $\psi$ is sufficiently regular, the intrinsic derivatives $D_{j}^{\psi} \psi$ are the derivatives of $\psi$ along the integral curves of the vector fields $D_{j}^{\psi}$. We use a similar technique exploited in Proposition 3.7 in [2] in Heisenberg groups.

Proposition 5.6. Let $\mathcal{O}$ be open in $\mathbb{W}, A \in \mathcal{O}$ and $\hat{\psi}=(\psi, 0, \ldots, 0): \mathcal{O} \rightarrow \mathbb{V}$. For $j=2, \ldots, m$ and $\delta>0$ denote $\gamma^{j}:[-\delta, \delta] \rightarrow \mathcal{O}$ an integral curve of the vector field $D_{j}^{\psi}$ such that $\gamma^{j}(0)=A$.

If we assume that

$$
\hat{\psi} \text { is u.i.d. in } A \in \mathcal{O} \text { and } t \mapsto \psi\left(\gamma^{j}(t)\right) \text { is in } \mathbb{C}^{1}(-\delta, \delta) \text {, }
$$

then

$$
\lim _{t \rightarrow 0} \frac{\psi\left(\gamma^{j}(t)\right)-\psi(A)}{t}=\left(\nabla^{\hat{\psi}} \hat{\psi}\right)_{j}(A),
$$


where with $\left(\nabla^{\hat{\psi}} \hat{\psi}\right)_{j}$ we mean the $j$-th component of a representation of the intrinsic differential as in (19).

Proof. Fix $j=2, \ldots, m$. Let $A=(x, y) \in \mathcal{O}$ such that $\gamma^{j}(0)=A$. We know that $\gamma^{j}(t)=$ $\left(\gamma_{2}^{j}(t), \ldots, \gamma_{m+n}^{j}(t)\right)$ is given by

$$
\gamma_{h}^{j}(t)= \begin{cases}x_{h} & h=2, \ldots, m, h \neq j \\ x_{h}+t & h=j \\ y_{h}+\frac{1}{2} t \sum_{l=2}^{m} x_{l} b_{j l}^{h}+b_{j 1}^{h} \int_{0}^{t} \psi\left(\gamma^{j}(r)\right) d r & h=m+1, \ldots, m+n\end{cases}
$$

and then

$$
\begin{aligned}
\left\|\hat{\psi}\left(\gamma^{j}(t)\right)-\hat{\psi}(A)-d \hat{\psi}_{A}\left(A^{-1} \gamma^{j}(t)\right)\right\| & =\left|\psi\left(\gamma^{j}(t)\right)-\psi(A)-\left(\nabla^{\hat{\psi}} \hat{\psi}\right)_{j}(A) t\right| \\
\left\|\hat{\psi}(A)^{-1} \cdot A^{-1} \cdot \gamma^{j}(t) \cdot \hat{\psi}(A)\right\| & \leq C|t| .
\end{aligned}
$$

for an appropriate $C>1$. We notice that in the first equality we use the fact that $d \hat{\psi}_{A}$ acts only on the first components (see (19)) and that the homogeneous norm on the first layer is the Euclidean norm. Indeed we have

$$
\begin{aligned}
c_{1}^{-1}\left\|\hat{\psi}(A)^{-1} \cdot A^{-1} \cdot \gamma^{j}(t) \cdot \hat{\psi}(A)\right\| & \leq|t|+\sum_{s=1}^{n}\left|b_{j 1}^{s} \int_{0}^{t} \psi\left(\gamma^{j}(r)\right) d r-t b_{j 1}^{s} \psi(A)\right|^{1 / 2} \\
& =|t|+\sum_{s=1}^{n}\left|b_{j 1}^{s} \int_{0}^{t}\left(\psi\left(\gamma^{j}(r)\right)-\psi(A)\right) d r\right|^{1 / 2}
\end{aligned}
$$

Moreover for $h=m+1, \ldots, m+n$, the map $t \mapsto \gamma_{h}^{j}(t)$ is of class $\mathbb{C}^{2}$ (because of (32) and the hypothesis that $t \mapsto \psi\left(\gamma^{j}(t)\right)$ is $\left.\mathbb{C}^{1}\right)$, hence for all $s=1, \ldots, n$

$$
b_{j 1}^{s} \int_{0}^{t}\left(\psi\left(\gamma^{j}(r)\right)-\psi(A)\right) d r=O\left(t^{2}\right) .
$$

Hence (33) holds with a appropriate $C=C\left(c_{1}\right)>0$, and from (33) we get

$$
\frac{\left|\psi\left(\gamma^{j}(t)\right)-\psi(A)-\left(\nabla^{\hat{\psi}} \hat{\psi}\right)_{j}(A) t\right|}{t} \leq C \frac{\left|\hat{\psi}\left(\gamma^{j}(t)\right)-\hat{\psi}(A)-d \hat{\psi}_{A}\left(A^{-1} \gamma^{j}(t)\right)\right|}{\left\|\hat{\psi}(A)^{-1} \cdot A^{-1} \cdot \gamma^{j}(t) \cdot \hat{\psi}(A)\right\|}
$$

where $d \hat{\psi}_{A}$ is the intrinsic differential of $\hat{\psi}$ at $A$. By letting $t \rightarrow 0$ and using the assumption of intrinsic differentiability of $\hat{\psi}$ at $A$, we obtain the thesis.

5.3. Broad* solutions and $\nabla^{\psi}$-exponential maps. In this section we prove a converse of the previous results. In sections 3 and 4 we proved that if $\operatorname{graph}(\hat{\psi})$ is a $\mathbb{G}$-regular hypersurface then the intrinsic derivatives $D_{j}^{\psi} \psi$ are continuous. Here we show that if $\psi: \mathcal{O} \subset \mathbb{R}^{m+n-1} \rightarrow \mathbb{R}$ is a continuous solution in an open set $\mathcal{O}$ of the non linear first order system

$$
\left(D_{2}^{\psi} \psi, \ldots, D_{m}^{\psi} \psi\right)=w
$$

where $w: \mathcal{O} \rightarrow \mathbb{R}^{m-1}$ is a given continuous function then $\hat{\psi}: \hat{\mathcal{O}} \rightarrow \mathbb{V}$ is uniformly intrinsic differentiable in $\hat{\mathcal{O}}$ and consequently its graph is a $\mathbb{G}$-regular hypersurface. The main equivalence result is contained in Theorem 5.8.

In order to state the equivalence result we have to be precise about the meaning of being a solution of (34). To this aim we recall a notion of generalized solutions of systems of this kind. These generalized solutions, denoted broad* solutions were introduced and studied for the system (34) inside Heisenberg groups in [2, 5]. For a more complete bibliography we refer to the bibliography in [2]. 
Definition 5.3. Let $\mathcal{O} \subset \mathbb{R}^{m+n-1}$ be open and $w:=\left(w_{2}, \ldots, w_{m}\right): \mathcal{O} \rightarrow \mathbb{R}^{m-1}$ a continuous function. With the notations of Definition 5.2 we say that $\psi \in \mathbb{C}(\mathcal{O}, \mathbb{R})$ is a broad ${ }^{*}$ solution in $\mathcal{O}$ of the system

$$
\left(D_{2}^{\psi} \psi, \ldots, D_{m}^{\psi} \psi\right)=w
$$

if for every $A \in \mathcal{O}$ there are $0<\delta_{2}<\delta_{1}$ and $m-1 \operatorname{maps} \exp _{A}\left(\cdot D_{j}^{\psi}\right)(\cdot)$

$$
\begin{array}{r}
\exp _{A}\left(\cdot D_{j}^{\psi}\right)(\cdot):\left[-\delta_{2}, \delta_{2}\right] \times \mathcal{I}\left(A, \delta_{2}\right) \rightarrow \mathcal{I}\left(A, \delta_{1}\right) \\
(t, B) \mapsto \exp _{A}\left(t D_{j}^{\psi}\right)(B)
\end{array}
$$

for $j=2, \ldots, m$, where $\mathcal{I}(A, \delta):=\mathcal{U}(A, \delta) \cap \mathbb{W}$ and $\mathcal{I}\left(A, \delta_{1}\right) \subset \mathcal{O}$. Moreover these maps, called exponential maps of the vector fields $D_{2}^{\psi}, \ldots, D_{m}^{\psi}$, are required to have the following properties

$$
t \mapsto \gamma_{B}^{j}(t):=\exp _{A}\left(t D_{j}^{\psi}\right)(B) \in \mathbb{C}^{1}\left(\left[-\delta_{2}, \delta_{2}\right], \mathbb{R}^{m+n-1}\right)
$$

for all $B \in \mathcal{I}\left(A, \delta_{2}\right)$ and

$$
\begin{gathered}
\left\{\begin{array}{l}
\dot{\gamma}_{B}^{j}=D_{j}^{\psi} \circ \gamma_{B}^{j} \\
\gamma_{B}^{j}(0)=B
\end{array}\right. \\
\psi\left(\gamma_{B}^{j}(t)\right)-\psi\left(\gamma_{B}^{j}(0)\right)=\int_{0}^{t} w_{j}\left(\gamma_{B}^{j}(r)\right) d r \quad \text { for } t \in\left[-\delta_{2}, \delta_{2}\right]
\end{gathered}
$$

once more for all $B \in \mathcal{I}\left(A, \delta_{2}\right)$.

Remark 5.7. If the exponential maps of $D_{2}^{\psi}, \ldots, D_{m}^{\psi}$ at $A$ exist, then the map

$$
\left[-\delta_{2}, \delta_{2}\right] \ni t \longmapsto \psi\left(\exp _{A}\left(t D_{j}^{\psi}\right)(B)\right)
$$

is of class $\mathbb{C}^{1}$ for every $j=2, \ldots, m$ and for each $B \in \mathcal{I}_{\delta_{2}}(A)$.

Theorem 5.8. Let $\mathbb{G}$ be a group of class $\mathcal{B}$ and that

$$
\mathbb{V}:=\left\{\left(x_{1}, 0 \ldots, 0\right)\right\}, \quad \mathbb{W}:=\left\{\left(0, x_{2}, \ldots, x_{m+n}\right)\right\} .
$$

Let $\mathcal{O}$ open in $\mathbb{R}^{m+n-1}$ and $\psi: \mathcal{O} \rightarrow \mathbb{R}, \hat{\psi}: \hat{\mathcal{O}} \rightarrow \mathbb{V}$ be a continuous function. Then the following conditions are equivalent:

(1) $\operatorname{graph}(\hat{\psi})$ is a $\mathbb{G}$-regular hypersurface, i.e. for all $A \in \operatorname{graph}(\hat{\psi})$ there is $r=r(A)>0$ and $\left.f \in \mathbb{C}_{\mathbb{G}}^{1}(\mathcal{U}(A, r)), \mathbb{R}\right)$ with $X_{1} f>0$ such that

$$
\operatorname{graph}(\hat{\psi}) \cap \mathcal{U}(A, r)=\{P: f(P)=0\}
$$

(2) $\hat{\psi}$ is u.i.d. in $\hat{\mathcal{O}}$.

(3) there exists $w \in \mathbb{C}\left(\mathcal{O}, \mathbb{R}^{m-1}\right)$ such that, if we consider the distribution $D_{j}^{\psi} \psi$ defined as in (29), we have $\left(D_{2}^{\psi} \psi, \ldots, D_{m}^{\psi} \psi\right)=w$ in the sense of distribution. Moreover, for $0<\epsilon<1$, there is a family of functions $\psi_{\epsilon} \in \mathbb{C}^{1}(\mathcal{O}, \mathbb{R})$ such that for all $\mathcal{O}^{\prime} \Subset \mathcal{O}$,

$$
\psi_{\epsilon} \rightarrow \psi \quad \text { and } \quad D_{j}^{\psi_{\epsilon}} \psi_{\epsilon} \rightarrow w
$$

for $j=2, \ldots, m$, uniformly on $\mathcal{O}^{\prime}$ as $\epsilon \rightarrow 0^{+}$.

(4) $\psi \in h_{\text {loc }}^{1 / 2}(\mathcal{O})$ (see Proposition $3.7(2)$ ) and there exists $w \in \mathbb{C}\left(\mathcal{O}, \mathbb{R}^{m-1}\right.$ ) such that $\psi$ is a broad $^{*}$ solution of

$$
\left(D_{2}^{\psi} \psi, \ldots, D_{m}^{\psi} \psi\right)=w
$$

in $\mathcal{O}$. 
Proof. We split the proof in several steps. We should cite as inspiration Theorem 5.1 in 2 for the proof of $(\mathbf{2}) \Longrightarrow(3)$; Lemma 5.6 in [2] for the proof of $(\mathbf{3}) \Longrightarrow(4)$ and Lemma 5.7 in [2] for the proof of $(4) \Longrightarrow(2)$.

$(\mathbf{1}) \Longleftrightarrow(\mathbf{2})$ see Theorem 4.1 .

$(2) \Longrightarrow(3)$

In the proof of Proposition 5.4 (i), we prove that for all $A \in \mathcal{O}$ there are $\delta=\delta(A)>0$ with $\mathcal{I}(A, \delta):=\mathcal{U}(A, \delta) \cap \mathcal{O}$, a map $w \in \mathbb{C}\left(\mathcal{O}, \mathbb{R}^{m-1}\right)$ and a family $\left(\psi_{\epsilon}\right)_{\epsilon>0} \subset \mathbb{C}^{1}(\mathcal{I}(A, \delta), \mathbb{R})$ such that

$$
\psi_{\epsilon} \rightarrow \psi \text { and } D_{j}^{\psi_{\epsilon}} \psi_{\epsilon} \rightarrow w \quad \text { uniformly on } \mathcal{I}(A, \delta) \text {, as } \epsilon \rightarrow 0 \text {. }
$$

Moreover, Proposition 5.4 (i) states that the distribution $D^{\psi} \psi$ as (29) is equal to $w$ in the distributional sense.

From what proved up to now we know that for all $B \in \mathcal{O}$ there is $\delta=\delta(B)>0$ such that $\mathcal{I}(B, \delta) \Subset \mathcal{O}$ and there is family $\mathcal{A}=\mathcal{A}(B)$ of $\mathbb{C}^{1}$ functions defined as $\mathcal{A}:=\left\{\psi_{\epsilon, B}: \mathcal{I}(B, \delta) \rightarrow\right.$ $\mathbb{R}\}_{0<\epsilon<1}$ such that (35) holds.

Now let $\hat{\mathcal{F}}:=\{\operatorname{Int}(\mathcal{I}(B, \delta)): B \in \mathcal{O}\}$ be open covering of $\mathcal{O}$. Then there exists a locally finite countable subcovering $\mathcal{F}$ of $\hat{\mathcal{F}}$ and $\left\{\theta_{h}: \mathcal{O} \rightarrow \mathbb{R} \mid h \in \mathbb{N}\right\}$ a partition of the unity subordinate to $\mathcal{F}$.

Let $\psi_{\epsilon, B_{h}} \in \mathcal{A}$. We consider $\psi_{\epsilon, h}:=\psi_{\epsilon, B_{h}}: \mathbb{R}^{m+n-1} \rightarrow \mathbb{R}$ where from now on, if necessary, we use the convention of extending functions by letting them vanish outside an enlargement of their domain, to preserve the regularity. Let $\psi_{\epsilon}:=\sum_{h=1}^{\infty} \theta_{h} \psi_{\epsilon, h}$; by construction $\psi_{\epsilon} \in \mathbb{C}^{1}(\mathcal{O}, \mathbb{R})$ and

$$
D_{j}^{\psi_{\epsilon}} \psi_{\epsilon}=\sum_{h=1}^{\infty}\left(\psi_{\epsilon, h} D_{j}^{\psi_{\epsilon}} \theta_{h}+\theta_{h} D_{j}^{\psi_{\epsilon}} \psi_{\epsilon, h}\right) \quad \text { on } \mathcal{O}
$$

Because the partition is locally finite, there are only a finite number of $h_{1}, \ldots, h_{l}$ such that $\overline{\mathcal{O}^{\prime}} \cap$ $\operatorname{spt} \theta_{h_{\tau}} \neq \emptyset$ for each $\tau=1, \ldots, l$ and $\overline{\mathcal{O}^{\prime}} \subset \bigcup_{\tau=1}^{l} \operatorname{spt} \theta_{h_{\tau}}$. Then

$$
\begin{gathered}
\psi_{\epsilon}=\sum_{\tau=1}^{l} \theta_{h_{\tau}} \psi_{\epsilon, h_{\tau}} \text { and } \psi=\sum_{\tau=1}^{l} \theta_{h_{\tau}} \psi \text { on } \overline{\mathcal{O}^{\prime}} \\
D_{j}^{\psi_{\epsilon}} \psi_{\epsilon}=\sum_{\tau=1}^{l}\left(\psi_{\epsilon, h_{\tau}} D_{j}^{\psi_{\epsilon}} \theta_{h_{\tau}}+\theta_{h_{\tau}} D_{j}^{\psi_{\epsilon}} \psi_{\epsilon, h_{\tau}}\right) \text { on } \overline{\mathcal{O}^{\prime}}
\end{gathered}
$$

Putting together the last equalities and (35) we get

$$
\psi_{\epsilon} \rightarrow \psi \quad \text { and } \quad D_{j}^{\psi_{\epsilon}} \psi_{\epsilon} \rightarrow \sum_{\tau=1}^{l}\left(\psi D_{j}^{\psi} \theta_{h_{\tau}}+\theta_{h_{\tau}} D_{j}^{\psi} \psi\right)=w_{j} \quad \text { uniformly on } \overline{\mathcal{O}^{\prime}}, \quad \text { as } \epsilon \rightarrow 0
$$

This completes the proof of the implication $(2) \Longrightarrow(3)$.

(3) $\Longrightarrow(4)$

The proof of $\psi \in h_{l o c}^{1 / 2}(\mathcal{O})$ is the content of Theorem [5.9.

To prove that $\psi$ is a broad* solution we have to show that for each $A \in \mathcal{O}$ there exist $\delta_{1}, \delta_{2}>0$ with $\delta_{1}>\delta_{2}$ such that for $j=2, \ldots, m$ there is an exponential map $\exp _{A}\left(t D_{j}^{\psi}\right)(B) \in \mathcal{I}\left(A, \delta_{1}\right) \Subset \mathcal{O}$ for all $(t, B) \in\left[-\delta_{2}, \delta_{2}\right] \times \mathcal{I}\left(A, \delta_{2}\right)$; moreover,

for all $B \in \mathcal{I}\left(A, \delta_{2}\right)$.

$$
w_{j}(B)=\frac{d}{d t} \psi\left(\exp _{A}\left(t D_{j}^{\psi}\right)(B)\right)_{\mid t=0}
$$

Fix $j=2, \ldots, m$. For $\epsilon>0$ we consider the Cauchy problem

$$
\left\{\begin{array}{c}
\dot{\gamma}_{B, \epsilon}^{j}(t)=D_{j}^{\psi_{\epsilon}}\left(\gamma_{B, \epsilon}^{j}(t)\right) \\
\gamma_{B, \epsilon}^{j}(0)=B \\
27
\end{array}\right.
$$


which has a solution $\gamma_{\epsilon}:\left[-\delta_{2}(\epsilon), \delta_{2}(\epsilon)\right] \times \overline{\mathcal{I}\left(A, \delta_{2}(\epsilon)\right)} \rightarrow \overline{\mathcal{I}\left(A, \delta_{1}\right)}$. By Peano's estimate on the existence time for solutions of ordinary differential equations we get that $\delta_{2}(\epsilon)$ is greater than $C /\left\|\psi_{\epsilon}\right\|_{\mathcal{L}^{\infty}\left(\mathcal{I}\left(A, \delta_{1}(\epsilon)\right)\right)}$, with $C$ depending only on $\delta_{1}$. So it is sufficient to take $\delta_{2}>0$ such that $\delta_{2} \leq \delta_{2}(\epsilon)$ for all $\epsilon$. Because $\gamma_{\epsilon}$ are uniformly continuous on the compact $\left[-\delta_{2}, \delta_{2}\right] \times \overline{\mathcal{I}\left(A, \delta_{2}\right)}$, by Ascoli-Arzelá Theorem, we have a sequence $\left(\epsilon_{h}\right)_{h}$ such that $\epsilon_{h} \rightarrow 0$ as $h \rightarrow \infty$ and $\gamma_{\epsilon_{h}} \rightarrow \gamma$ uniformly on $\left[-\delta_{2}, \delta_{2}\right] \times \overline{\mathcal{I}\left(A, \delta_{2}\right)}$. Obviously,

$$
\begin{gathered}
\gamma_{B, \epsilon_{h}}^{j}(t)=B+\int_{0}^{t} D_{j}^{\psi_{\epsilon_{h}}}\left(\gamma_{B, \epsilon_{h}}^{j}(r)\right) d r \\
\psi_{\epsilon_{h}}\left(\gamma_{B, \epsilon_{h}}^{j}(t)\right)-\psi_{\epsilon_{h}}\left(\gamma_{B, \epsilon_{h}}^{j}(0)\right)=\int_{0}^{t} D_{j}^{\psi_{\epsilon_{h}}} \psi_{\epsilon_{h}}\left(\gamma_{B, \epsilon_{h}}^{j}(r)\right) d r
\end{gathered}
$$

and for $h \rightarrow \infty$ using that all the involved convergences are uniform we conclude

$$
\begin{gathered}
\gamma_{B}^{j}(t)=B+\int_{0}^{t} D_{j}^{\psi}\left(\gamma_{B}^{j}(r)\right) d r \\
\psi\left(\gamma_{B}^{j}(t)\right)-\psi\left(\gamma_{B}^{j}(0)\right)=\int_{0}^{t} w_{j}\left(\gamma_{B}^{j}(r)\right) d r
\end{gathered}
$$

i.e. the conditions of the Definition 5.3 are satisfied.

$(4) \Longrightarrow(2)$.

Let us fix $A=(\bar{x}, \bar{y}) \in \mathcal{O}$. First let $B=(x, y), B^{\prime}=\left(x^{\prime}, y^{\prime}\right) \in \mathcal{I}(A, \delta)$ for a sufficiently small $\delta>0$.

Here we can not integrate along the vector field $D_{j}^{\psi}$; however this obstacle can be solved using the exponential maps, more precisely by posing

$$
B_{i}:=\exp _{A}\left(\bar{D}_{i}\right)\left(B_{i-1}\right) \quad \text { for } i=2, \ldots, m
$$

where $\bar{D}:=\left(\bar{D}_{2}, \ldots, \bar{D}_{m}\right)$ is the family of vector fields given by $\bar{D}_{j}=\left(x_{j}^{\prime}-x_{j}\right) D_{j}^{\psi}$ for $j \in\{2, \ldots, m\}$. A computation gives that

$$
B_{j}=\left(x_{2}^{\prime}, \ldots, x_{j}^{\prime}, x_{j+1}, \ldots, x_{m}, y^{B_{j}}\right)
$$

with

$$
\begin{aligned}
y_{s}^{B_{j}} & =y_{s}+\sum_{l=2}^{j}\left(-b_{1 l}^{s} \int_{0}^{x_{l}^{\prime}-x_{l}} \psi\left(\exp _{A}\left(r D_{l}^{\psi}\right)\left(B_{l-1}\right)\right) d r+\frac{1}{2}\left(x_{l}^{\prime}-x_{l}\right)\left(\sum_{i=2}^{l} x_{i}^{\prime} b_{l i}^{s}+\sum_{i=l+1}^{m} x_{i} b_{l i}^{s}\right)\right) \\
& =y_{s}^{B_{j-1}}-b_{1 j}^{s} \int_{0}^{x_{j}^{\prime}-x_{j}} \psi\left(\exp _{A}\left(r D_{j}^{\psi}\right)\left(B_{j-1}\right)\right) d r+\frac{1}{2}\left(x_{j}^{\prime}-x_{j}\right)\left(\sum_{i=2}^{j} x_{i}^{\prime} b_{j i}^{s}+\sum_{i=j+1}^{m} x_{i} b_{j i}^{s}\right)
\end{aligned}
$$

for $s=1, \ldots, n$. Observe that $B_{2}, \ldots, B_{m}$ are well defined for a sufficiently small $\delta$. Because $\psi$ is of class $\mathbb{C}^{1}$ (see Remark 5.7) we have

$$
\begin{aligned}
\psi\left(B^{\prime}\right)-\psi(B) & =\left[\psi\left(B^{\prime}\right)-\psi\left(B_{m}\right)\right]+\sum_{l=2}^{m}\left[\psi\left(B_{l}\right)-\psi\left(B_{l-1}\right)\right] \\
& =\left[\psi\left(B^{\prime}\right)-\psi\left(B_{m}\right)\right]+\sum_{l=2}^{m}\left(\bar{D}_{l} \psi\left(B_{l-1}\right)+o\left(\left|x_{l}^{\prime}-x_{l}\right|\right)\right)
\end{aligned}
$$


Notice that, in the last equality, we used the fact

$$
\begin{aligned}
& \sum_{l=2}^{m}\left(\psi\left(B_{l}\right)-\psi\left(B_{l-1}\right)\right)= \sum_{l=2}^{m}\left(\int_{0}^{1}\left(x_{l}^{\prime}-x_{l}\right) w_{l}\left(\exp _{A}\left(r \bar{D}_{l}\right)\left(B_{l-1}\right)\right) d r\right) \\
& \quad(\text { by the continuity of } w) \\
&=\sum_{l=2}^{m}\left(\left(x_{l}^{\prime}-x_{l}\right) w_{l}\left(B_{l-1}\right)+o\left(\left|x_{l}^{\prime}-x_{l}\right|\right)\right)
\end{aligned}
$$

Now, using again the continuity of $w$, we have

$$
\lim _{\delta \rightarrow 0} \frac{\sum_{l=2}^{m}\left(x_{l}^{\prime}-x_{l}\right) w_{l}\left(B_{l-1}\right)-\left\langle w(A), x^{\prime}-x\right\rangle}{\left|x^{\prime}-x\right|_{\mathbb{R}^{m-1}}}=0
$$

and so

$$
\begin{aligned}
\psi\left(B^{\prime}\right)-\psi(B)= & \psi\left(B^{\prime}\right)-\psi\left(B_{m}\right)+\left\langle w(A), x^{\prime}-x\right\rangle+\left(\sum_{l=2}^{m}\left(x_{l}^{\prime}-x_{l}\right) w_{l}\left(B_{l-1}\right)-\left\langle w(A), x^{\prime}-x\right\rangle\right) \\
& +o\left(\left|x^{\prime}-x\right|_{\mathbb{R}^{m-1}}\right) \\
= & \psi\left(B^{\prime}\right)-\psi\left(B_{m}\right)+\left\langle w(A), x^{\prime}-x\right\rangle+o\left(\left\|\hat{\psi}(B)^{-1} B^{-1} B^{\prime} \hat{\psi}(B)\right\|\right) .
\end{aligned}
$$

Consequently, it is sufficient to show that $\psi\left(B^{\prime}\right)-\psi\left(B_{m}\right)=o\left(\left\|\hat{\psi}(B)^{-1} B^{-1} B^{\prime} \hat{\psi}(B)\right\|\right)$. First we observe that

$$
\frac{\left|\psi\left(B^{\prime}\right)-\psi\left(B_{m}\right)\right|}{\left\|\hat{\psi}(B)^{-1} B^{-1} B^{\prime} \hat{\psi}(B)\right\|} \leq C_{\psi}(\delta) \frac{\left|y^{\prime}-y^{B_{m}}\right|_{\mathbb{R}^{n}}^{1 / 2}}{\left\|\hat{\psi}(B)^{-1} B^{-1} B^{\prime} \hat{\psi}(B)\right\|}
$$

with

$$
C_{\psi}(\delta):=\sup \left\{\frac{\left|\psi\left(A^{\prime}\right)-\psi\left(A^{\prime \prime}\right)\right|}{\left|A^{\prime}-A^{\prime \prime}\right|_{\mathbb{R}^{m+n-1}}^{1 / 2}}: A^{\prime} \neq A^{\prime \prime}, A^{\prime}, A^{\prime \prime} \in \mathcal{I}(A, \delta)\right\}
$$

We know also that $\lim _{\delta \rightarrow 0} C_{\psi}(\delta)=0$ because $\psi \in h_{\text {loc }}^{1 / 2}(\mathcal{O})$. So it is evident that remains to prove $\left|y^{\prime}-y^{B_{m}}\right|_{\mathbb{R}^{n}}^{1 / 2} /\left\|\hat{\psi}(B)^{-1} B^{-1} B^{\prime} \hat{\psi}(B)\right\|$ is bounded in a proper neighborhood of $A$. 
If we put $\mathcal{B}_{M}=\max \left\{b_{i j}^{s} \mid i, j=1, \ldots, m, s=1, \ldots, n\right\}$ (observe that $\mathcal{B}_{M}>0$ because the matrices $\mathcal{B}^{(s)}$ are skew-symmetric), then

$$
\begin{aligned}
\left|y^{\prime}-y^{B_{m}}\right|_{\mathbb{R}^{n}} \leq & \sum_{s=1}^{n} \mid y_{s}^{\prime}-y_{s}+\sum_{l=2}^{m}\left(b_{1 l}^{s} \int_{0}^{x_{l}^{\prime}-x_{l}} \psi\left(\exp _{A}\left(r D_{l}^{\psi}\right)\left(B_{l-1}\right)\right) d r+\right. \\
& \left.-\frac{1}{2}\left(x_{l}^{\prime}-x_{l}\right)\left(\sum_{i=2}^{l} x_{i}^{\prime} b_{l i}^{s}+\sum_{i=l+1}^{m} x_{i} b_{l i}^{s}\right)\right) \mid \\
\leq & \sum_{s=1}^{n}\left|y_{s}^{\prime}-y_{s}+\psi(B) \sum_{l=2}^{m}\left(x_{l}^{\prime}-x_{l}\right) b_{1 l}^{s}-\frac{1}{2}\left\langle\mathcal{B}^{(s)} x, x^{\prime}-x\right\rangle\right| \\
& +\sum_{s=1}^{n}\left|-\frac{1}{2}\left(x_{l}^{\prime}-x_{l}\right)\left(\sum_{i=2}^{l} x_{i}^{\prime} b_{l i}^{s}+\sum_{i=l+1}^{m} x_{i} b_{l i}^{s}\right)+\frac{1}{2}\left\langle\mathcal{B}^{(s)} x, x^{\prime}-x\right\rangle\right| \\
& +\sum_{s=1}^{n}\left|-\psi(B) \sum_{l=2}^{m}\left(x_{l}^{\prime}-x_{l}\right) b_{1 l}^{s}+\sum_{l=2}^{m} b_{1 l}^{s} \int_{0}^{x_{l}^{\prime}-x_{l}} \psi\left(\exp _{A}\left(r D_{l}^{\psi}\right)\left(B_{l-1}\right)\right) d r\right| \\
\leq & c_{1}\left\|\hat{\phi}(i(B))^{-1} i(B)^{-1} i\left(B^{\prime}\right) \hat{\phi}(i(B))\right\|^{2}+\frac{1}{2} n \mathcal{B}_{M}\left|x^{\prime}-x\right|_{\mathbb{R}^{m-1}}^{2} \\
& +\sum_{s=1}^{n}\left|-\psi(B) \sum_{l=2}^{m}\left(x_{l}^{\prime}-x_{l}\right) b_{1 l}^{s}+\sum_{l=2}^{m} b_{1 l}^{s} \int_{0}^{x_{l}^{\prime}-x_{l}} \psi\left(\exp _{A}\left(r D_{l}^{\psi}\right)\left(B_{l-1}\right)\right) d r\right|
\end{aligned}
$$

where $c_{1}$ is given by (25). Note that we have used

$$
\begin{aligned}
\left|\frac{1}{2}\left\langle\mathcal{B}^{(s)} x, x^{\prime}-x\right\rangle-\frac{1}{2}\left(x_{l}^{\prime}-x_{l}\right)\left(\sum_{i=2}^{l} x_{i}^{\prime} b_{l i}^{s}+\sum_{i=l+1}^{m} x_{i} b_{l i}^{s}\right)\right| \\
=\left|-\frac{1}{2}\left(x_{l}^{\prime}-x_{l}\right)\left(\sum_{i=2}^{l} x_{i}^{\prime} b_{l i}^{s}+\sum_{i=l+1}^{m} x_{i} b_{l i}^{s}-\sum_{i=2}^{m} x_{i} b_{l i}^{s}\right)\right| \leq \frac{1}{2} \mathcal{B}_{M}\left|x^{\prime}-x\right|_{\mathbb{R}^{m-1}}^{2} .
\end{aligned}
$$

Finally, we consider the last term of (36)

$$
\begin{aligned}
\sum_{s=1}^{n} \mid & -\psi(B) \sum_{l=2}^{m}\left(x_{l}^{\prime}-x_{l}\right) b_{1 l}^{s}+\sum_{l=2}^{m} b_{1 l}^{s} \int_{0}^{x_{l}^{\prime}-x_{l}} \psi\left(\exp _{A}\left(r D_{l}^{\psi}\right)\left(B_{l-1}\right)\right) d r \mid \\
& \leq R_{1}\left(B, B^{\prime}\right)+R_{2}\left(B, B^{\prime}\right)
\end{aligned}
$$

where

$$
\begin{aligned}
& R_{1}\left(B, B^{\prime}\right):=\sum_{s=1}^{n} \sum_{l=2}^{m}\left|b_{1 l}^{s} \int_{0}^{x_{l}^{\prime}-x_{l}} \psi\left(\exp _{A}\left(r D_{l}^{\psi}\right)\left(B_{l-1}\right)\right) d r-b_{1 l}^{s} \psi\left(B_{l-1}\right)\left(x_{l}^{\prime}-x_{l}\right)\right| \\
& R_{2}\left(B, B^{\prime}\right):=\sum_{s=1}^{n}\left|\sum_{l=2}^{m} b_{1 l}^{s}\left(x_{l}^{\prime}-x_{l}\right)\left(\psi\left(B_{l-1}\right)-\psi(B)\right)\right|
\end{aligned}
$$

We would show that there exist $C_{1}, C_{2}>0$ such that

$$
\begin{aligned}
& R_{1}\left(B, B^{\prime}\right) \leq C_{1}\left|x^{\prime}-x\right|_{\mathbb{R}^{m-1}}^{2} \\
& R_{2}\left(B, B^{\prime}\right) \leq C_{2}\left|x^{\prime}-x\right|_{\mathbb{R}^{m-1}}^{2}
\end{aligned}
$$

for all $B, B^{\prime} \in \mathcal{I}(A, \delta)$, and consequently

$$
\left|y^{\prime}-y^{B_{m}}\right|_{\mathbb{R}^{n}} \leq c_{1}\left\|\hat{\phi}(i(B))^{-1} i(B)^{-1} i\left(B^{\prime}\right) \hat{\phi}(i(B))\right\|^{2}+\left(\frac{1}{2} n \mathcal{B}_{M}+C_{1}+C_{2}\right)\left|x^{\prime}-x\right|_{\mathbb{R}^{m-1}}^{2}
$$


Hence there is $C_{3}>0$ such that

$$
\left|y^{\prime}-y^{B_{m}}\right|_{\mathbb{R}^{n}}^{1 / 2} \leq C_{3}\left\|\hat{\psi}(B)^{-1} B^{-1} B^{\prime} \hat{\psi}(B)\right\|
$$

which is the thesis.

We start to consider $R_{1}\left(B, B^{\prime}\right)$. Fix $l=2, \ldots, m$ and $s=1, \ldots, n$. For $t \in[-\delta, \delta]$ we define

$$
g_{l}^{s}(t):=b_{1 l}^{s}\left(\int_{0}^{t} \psi\left(\exp _{A}\left(r D_{l}^{\psi}\right)\left(B_{l-1}\right)\right) d r-t \psi\left(B_{l-1}\right)\right)
$$

working as in Proposition 5.6 we show the existence of $C_{l}^{s}>0$ such that

$$
\left|g_{l}^{s}(t)\right| \leq C_{l}^{s} t^{2}, \quad \forall t \in[-\delta, \delta]
$$

So set $t=x_{l}^{\prime}-x_{l}$ we get

$$
\left|g_{l}^{s}\left(x_{l}^{\prime}-x_{l}\right)\right| \leq C_{l}^{s}\left(x_{l}^{\prime}-x_{l}\right)^{2}
$$

and consequently (37) follows from

$$
\sum_{l=2}^{m} \sum_{s=1}^{n}\left|g_{l}^{s}\left(x_{l}^{\prime}-x_{l}\right)\right| \leq \sum_{l=2}^{m} \sum_{s=1}^{n} C_{l}^{s}\left(x_{l}^{\prime}-x_{l}\right)^{2} \leq C_{1}\left|x^{\prime}-x\right|_{\mathbb{R}^{m-1}}^{2} .
$$

Now we consider $R_{2}\left(B, B^{\prime}\right)$. Observe that

$$
\begin{aligned}
\sum_{s=1}^{n} \mid \sum_{l=2}^{m} b_{1 l}^{s}\left(x_{l}^{\prime}\right. & \left.-x_{l}\right)\left(\psi\left(B_{l-1}\right)-\psi(B)\right)\left|\leq n \mathcal{B}_{M} \sum_{l=3}^{m}\right| x_{l}^{\prime}-x_{l}|| \psi\left(B_{l-1}\right)-\psi(B) \mid \\
& \leq n \mathcal{B}_{M} \sum_{l=3}^{m}\left|x_{l}^{\prime}-x_{l}\right|\left(\sum_{i=2}^{l-1}\left|\psi\left(B_{i}\right)-\psi\left(B_{i-1}\right)\right|\right) \\
& \leq n \mathcal{B}_{M} \sum_{l=3}^{m}\left|x_{l}^{\prime}-x_{l}\right|\left(\sum_{i=2}^{l-1}\left|\int_{0}^{1}\left(x_{i}^{\prime}-x_{i}\right) w_{i}\left(\exp _{A}\left(r \bar{D}_{i}\right)\left(B_{i-1}\right)\right) d r\right|\right) \\
& \leq n \mathcal{B}_{M} \sum_{l=3}^{m}\left|x_{l}^{\prime}-x_{l}\right|\left(\sum_{i=2}^{l-1}\left|\left(x_{i}^{\prime}-x_{i}\right)\left(w_{i}\left(B_{i-1}\right)+o(1)\right)\right|\right) \\
& \leq n \mathcal{B}_{M} C\left|x^{\prime}-x\right|_{\mathbb{R}^{m-1}}^{2}
\end{aligned}
$$

Then (38) follows with $C_{2}:=\frac{1}{2} n \mathcal{B}_{M} C$. As a consequence the (4) $\Longrightarrow(2)$ is proved.

We prove now that the solutions of the system $\left(D_{2}^{\psi} \psi, \ldots, D_{m}^{\psi} \psi\right)=w$ when $w \in \mathbb{C}\left(\mathcal{O}, \mathbb{R}^{m-1}\right)$ are Hölder continuous. We use the similar technique exploited in Theorem 5.8 and Theorem 5.9 in [2] in the context of Heisenberg groups.

Theorem 5.9. Let $\psi \in \mathbb{C}(\mathcal{O}, \mathbb{R})$ where $\mathcal{O}$ is open in $\mathbb{R}^{m+n-1}$. Assume that there exist $w:=$ $\left(w_{2}, \ldots, w_{m}\right) \in \mathbb{C}\left(\mathcal{O}, \mathbb{R}^{m-1}\right)$ and a family $\left(\psi_{\epsilon}\right)_{\epsilon>0} \subset \mathbb{C}^{1}(\mathcal{O}, \mathbb{R})$ such that, for any open $\mathcal{O}^{\prime} \Subset \mathcal{O}$,

$$
\psi_{\epsilon} \rightarrow \psi \quad \text { and } \quad D^{\psi_{\epsilon}} \psi_{\epsilon} \rightarrow w \quad \text { uniformly on } \mathcal{O}^{\prime} \text {, as } \epsilon \rightarrow 0^{+} \text {. }
$$

Then, for $\mathcal{O}^{\prime} \Subset \mathcal{O}^{\prime \prime} \Subset \mathcal{O}$ there exists $\alpha:(0,+\infty) \rightarrow[0,+\infty)$ depending only on $\mathcal{O}^{\prime \prime},\|\psi\|_{\mathcal{L}^{\infty}\left(\mathcal{O}^{\prime \prime}\right)}$, $\|w\|_{\mathcal{L}^{\infty}\left(\mathcal{O}^{\prime \prime}\right)}$, on $\mathcal{B}^{(1)}, \ldots, \mathcal{B}^{(n)}$ and on the modulus of continuity of $w$ on $\mathcal{O}^{\prime \prime}$ such that

$$
\lim _{r \rightarrow 0} \alpha(r)=0
$$

and

$$
\sup \left\{\frac{\left|\psi(A)-\psi\left(A^{\prime}\right)\right|}{\left|A-A^{\prime}\right|_{\mathbb{R}^{m+n-1}}^{1 / 2}}: A, A^{\prime} \in \mathcal{O}^{\prime}, 0<\left|A-A^{\prime}\right|_{\mathbb{R}^{m+n-1}} \leq r\right\} \leq \alpha(r) .
$$


Proof. It is sufficient to prove the theorem for $\psi \in \mathbb{C}^{1}(\mathcal{O}, \mathbb{R})$. Indeed from the uniform convergence of $\psi_{\epsilon}$ and $\left(D_{2}^{\psi_{\epsilon}} \psi_{\epsilon}, \ldots, D_{m}^{\psi_{\epsilon}} \psi_{\epsilon}\right)$, we can estimate $\left\|\psi_{\epsilon}\right\|_{\mathcal{L}^{\infty}\left(\mathcal{O}^{\prime}\right)}$, on $\left\|\left(D_{2}^{\psi_{\epsilon}} \psi_{\epsilon}, \ldots, D_{m}^{\psi_{\epsilon}} \psi_{\epsilon}\right)\right\|_{\mathcal{L}^{\infty}\left(\mathcal{O}^{\prime}\right)}$ uniformly in $\epsilon$ for any $\mathcal{O}^{\prime} \Subset \mathcal{O}$. Moreover the uniform convergence of $\left(D_{2}^{\psi_{\epsilon}} \psi_{\epsilon}, \ldots, D_{m}^{\psi_{\epsilon}} \psi_{\epsilon}\right)$ allows the choice of a modulus of continuity for $D_{j}^{\psi_{\epsilon}} \psi_{\epsilon}$ which is indipendent of $\epsilon$ for all $j$. Therefore there is $\alpha:(0,+\infty) \rightarrow[0,+\infty)$, not depending on $\epsilon$, such that (39) and (40) follow.

We are going to prove that for each point of $\mathcal{O}^{\prime}$ there are sufficiently small rectangular neighborhoods $\mathcal{I} \Subset \mathcal{I}^{\prime} \Subset \mathcal{O}$ and a function $\alpha:(0,+\infty) \rightarrow[0,+\infty)$ such that $\lim _{r \rightarrow 0} \alpha(r)=0$ and

$$
\sup \left\{\frac{\left|\psi(A)-\psi\left(A^{\prime}\right)\right|}{\left|A-A^{\prime}\right|_{\mathbb{R}^{m+n-1}}^{1 / 2}}: A, A^{\prime} \in \mathcal{I}, 0<\left|A-A^{\prime}\right|_{\mathbb{R}^{m+n-1}} \leq r\right\} \leq \alpha(r)
$$

By a standard covering argument the general statement follows.

Precisely we are going to prove (41) with $\alpha$ defined as

$$
\alpha(r):=\frac{3(1+h)}{\mathcal{B}_{m}} \delta\left(\max \left\{1, h^{2}\right\} r\right)+N r^{1 / 2}
$$

where if we put

$$
K:=\sup _{A=(x, y) \in \mathcal{I}^{\prime}} \sum_{i=2}^{m}\left|x_{i}\right|, \quad M:=\|\psi\|_{\mathcal{L}^{\infty}\left(\mathcal{I}^{\prime}\right)}, \quad N:=\|w\|_{\mathcal{L}^{\infty}\left(\mathcal{I}^{\prime}\right)}
$$

and $\beta:(0,+\infty) \rightarrow[0,+\infty)$, increasing, such that $\lim _{r \rightarrow 0^{+}} \beta(r)=0$ and

$$
\left|w(A)-w\left(A^{\prime}\right)\right| \leq \beta\left(\left|A-A^{\prime}\right|_{\mathbb{R}^{m+n-1}}\right) \quad \text { for all } A, A^{\prime} \in \mathcal{I}^{\prime}
$$

then $h:=\left(n \mathcal{B}_{M}(K+M)\right)^{1 / 2}, \mathcal{B}_{m}=\min \left\{\left|b_{i l}^{s}\right|: i, l=1, \ldots, m, s=1, \ldots, n\right.$ and $\left.b_{i l}^{s} \neq 0\right\}, \mathcal{B}_{M}=$ $\max \left\{b_{i l}^{s}: i, l=1, \ldots, m\right.$ and $\left.s=1, \ldots, n\right\}$ and

$$
\delta(r):=\max \left\{r^{1 / 4} ;\left(\mathcal{B}_{M} \beta\left(E r^{1 / 4}\right)\right)^{1 / 2}\right\} .
$$

Here $E>0$ is a constant such that $\left|y-y^{\prime}\right|_{\mathbb{R}^{n}}+\mathcal{B}_{M}(K+2 M)\left|y-y^{\prime}\right|_{\mathbb{R}^{n}}^{1 / 4} \leq E\left|y-y^{\prime}\right|_{\mathbb{R}^{n}}^{1 / 4}$ for all $(x, y),\left(x^{\prime}, y^{\prime}\right) \in \mathcal{I}^{\prime}$.

We split the proof in several steps.

Step 1. By standard considerations on ordinary differential equations, we know that for each point of $\mathcal{O}^{\prime}$ there are $r_{0}>0$ and rectangular neighborhoods $\mathcal{I} \Subset \mathcal{I}^{\prime} \Subset \mathcal{O}$ such that for all $A=$ $(x, y) \in \mathcal{I}$ there is a unique solution $\gamma_{A}^{j} \in \mathbb{C}^{1}\left(\left[x_{j}-r_{0}, x_{j}+r_{0}\right], \mathcal{I}^{\prime}\right)$ of the Cauchy problem

$$
\left\{\begin{array}{l}
\dot{\gamma}_{A}^{j}(t)=D_{j}^{\psi}\left(\gamma_{A}^{j}(t)\right)=X_{j} \psi\left(\gamma_{A}^{j}(t)\right)+\psi\left(\gamma_{A}^{j}(t)\right) \sum_{s=1}^{n} b_{j 1}^{s} Y_{s} \psi\left(\gamma_{A}^{j}(t)\right) \\
\gamma_{A}^{j}\left(x_{j}\right)=A
\end{array}\right.
$$

More precisely,

$$
\begin{aligned}
& \gamma_{A}^{j}(t)=\left(x_{2}, \ldots, x_{j-1}, t, x_{j+1}, \ldots, x_{m}, y_{1, A}^{j}(t), \ldots, y_{n, A}^{j}(t)\right), \quad \text { where } \\
& y_{s, A}^{j}(t)=y_{s}+\frac{1}{2}\left(t-x_{j}\right) \sum_{i=2}^{m} x_{i} b_{j i}^{s}+b_{j 1}^{s} \int_{x_{j}}^{t} \psi\left(\gamma_{A}^{j}(r)\right) d r, \quad \text { for } s=1, \ldots, n .
\end{aligned}
$$

Moreover observe that

$$
\frac{d^{2}}{d t^{2}} y_{s, A}^{j}(t)=\frac{d}{d t}\left[\frac{1}{2} \sum_{i=2}^{m} x_{i} b_{j i}^{s}+b_{j 1}^{s} \psi\left(\gamma_{A}^{j}(t)\right)\right]=b_{j 1}^{s} w_{j}\left(\gamma_{A}^{j}(t)\right) .
$$

Step 2. Assume $A, B \in \mathcal{I}$ (up to reducing $\mathcal{I}$ ) with $A=(x, y)$ and $B=(x, \bar{y})$. We prove that

$$
\frac{|\psi(A)-\psi(B)|}{|y-\bar{y}|_{\mathbb{R}^{n}}^{1 / 2}} \leq \frac{3}{\mathcal{B}_{m}} \delta
$$


where $\delta:=\delta\left(|y-\bar{y}|_{\mathbb{R}^{n}}\right)$. Suppose on the contrary that (45) is not true, i.e.

$$
\frac{|\psi(A)-\psi(B)|}{|y-\bar{y}|_{\mathbb{R}^{n}}^{1 / 2}}>\frac{3}{\mathcal{B}_{m}} \delta .
$$

Let $b_{j 1}^{s} \neq 0$ for some $s \in\{1, \ldots, n\}$ and $j=2, \ldots, m$. Observe that if $b_{j 1}^{s}=0$ for all $s \in\{1, \ldots, n\}$ and $j=2, \ldots, m$, then $w_{j}$ is smooth for each $j=2, \ldots, m$.

Let $\gamma_{A}^{j}$ and $\gamma_{B}^{j}$ with

$$
\gamma_{A}^{j}(t)=\left(x_{2}, \ldots, x_{j-1}, t, x_{j+1}, \ldots, x_{m}, y_{1}(t), \ldots, y_{n}(t)\right)
$$

and

$$
\gamma_{B}^{j}(t)=\left(x_{2}, \ldots, x_{j-1}, t, x_{j+1}, \ldots, x_{m}, \bar{y}_{1}(t), \ldots, \bar{y}_{n}(t)\right) .
$$

Suppose that $y_{s} \geq \bar{y}_{s}$ (for the other case it is sufficient to exchange the roles of $A$ and $B$ ). By (43) and (44), for $t \in\left[x_{j}-r_{0}, x_{j}+r_{0}\right]$ we have

$$
\begin{aligned}
& y_{s}(t)-\bar{y}_{s}(t)-\left(y_{s}-\bar{y}_{s}\right) \\
& =\int_{x_{j}}^{t}\left[\dot{y}_{s}\left(x_{j}\right)-\dot{\bar{y}}_{s}\left(x_{j}\right)+\int_{x_{j}}^{r^{\prime}}\left(\ddot{\bar{y}}_{s}(r)-\ddot{y}_{s}^{\prime}(r)\right) d r\right] d r^{\prime} \\
& =b_{j 1}^{s}\left(t-x_{j}\right)(\psi(A)-\psi(B))+b_{j 1}^{s} \int_{x_{j}}^{t} \int_{x_{j}}^{r^{\prime}}\left(w_{j}\left(\gamma_{A}^{j}(r)\right)-w_{j}\left(\gamma_{B}^{j}(r)\right)\right) d r d r^{\prime} .
\end{aligned}
$$

Now using the following facts

$$
\max _{r}\left|\dot{y}_{s}(r)\right|=\max _{r}\left|\frac{1}{2} \sum_{i=1}^{m} x_{i} b_{j i}^{s}+b_{j 1}^{s} \psi\left(\gamma_{A}^{j}(r)\right)\right| \leq \mathcal{B}_{M}\left(\frac{1}{2} K+M\right)
$$

and

$$
\begin{aligned}
& \left|\gamma_{A}^{j}(r)-\gamma_{B}^{j}(r)\right| \leq\left|\gamma_{A}^{j}\left(x_{j}\right)-\gamma_{B}^{j}\left(x_{j}\right)\right|+\left|r-x_{j}\right|\left(\max _{r}\left|\dot{y}_{s}(r)\right|+\max _{r}\left|\dot{\bar{y}}_{s}(r)\right|\right) \\
& \leq|A-B|_{\mathbb{R}^{m+n-1}}+\left|t-x_{j}\right|\left(\max _{r}\left|\dot{y}_{s}(r)\right|+\max _{\hat{r}}\left|\dot{\bar{y}}_{s}(r)\right|\right) \\
& \leq|y-\bar{y}|_{\mathbb{R}^{n}}+\left|t-x_{j}\right| \mathcal{B}_{M}(K+2 M)
\end{aligned}
$$

we obtain

$$
\begin{aligned}
& y_{s}(t)-\bar{y}_{s}(t)-\left(y_{s}-\bar{y}_{s}\right) \\
& \leq b_{j 1}^{s}\left(t-x_{j}\right)(\psi(A)-\psi(B))+\left|b_{j 1}^{s}\right|\left(t-x_{j}\right)^{2} \sup _{r} \beta\left(\left|\gamma_{A}^{j}(r)-\gamma_{B}^{j}(r)\right|\right) \\
& \leq b_{j 1}^{s}\left(t-x_{j}\right)(\psi(A)-\psi(B))+\left|b_{j 1}^{s}\right|\left(t-x_{j}\right)^{2} \beta\left(|y-\bar{y}|_{\mathbb{R}^{n}}+\left|t-x_{j}\right| \mathcal{B}_{M}(K+2 M)\right)
\end{aligned}
$$

So if $b_{j 1}^{s}(\psi(A)-\psi(B))>0$ put $t:=x_{j}-\frac{|y-\bar{y}|_{\mathbb{R}^{n}}^{1 / 2}}{\delta}$ in (46) and $t:=x_{j}+\frac{|y-\bar{y}|_{\mathbb{R}^{n}}^{1 / 2}}{\delta}$ otherwise. Observe that in both cases we conclude that

$$
-\left|b_{j 1}^{s}\right||\psi(A)-\psi(B)|<-3 \delta|y-\bar{y}|_{\mathbb{R}^{n}}^{1 / 2}
$$

If $|y-\bar{y}|_{\mathbb{R}^{n}}$ is "sufficiently small" $\gamma_{A}^{j}$ and $\gamma_{B}^{j}$ are well defined; it is sufficient to take $r_{0} \geq|y-\bar{y}|_{\mathbb{R}^{n}}^{1 / 4} \geq$ $|y-\bar{y}|_{\mathbb{R}^{n}}^{1 / 2} / \delta=\left|t-x_{j}\right|$ (it is exactly here that we reduce $\mathcal{I}$ ). Using (46), (47) and the definition of 
$\beta$ we obtain in both cases

$$
\begin{aligned}
y_{s}(t)-\bar{y}_{s}(t) \leq & y_{s}-\bar{y}_{s}+\left|b_{j 1}^{s}\right||y-\bar{y}|_{\mathbb{R}^{n}}^{1 / 2} \frac{-|\psi(A)-\psi(B)|}{\delta}+ \\
& +\frac{1}{\delta^{2}}\left|b_{j 1}^{s}\right||y-\bar{y}|_{\mathbb{R}^{n}} \beta\left(|y-\bar{y}|_{\mathbb{R}^{n}}+\mathcal{B}_{M}(K+2 M) \frac{|y-\bar{y}|_{\mathbb{R}^{n}}^{1 / 2}}{\delta}\right) \\
\leq & y_{s}-\bar{y}_{s}-3|y-\bar{y}|_{\mathbb{R}^{n}}^{1 / 2}|y-\bar{y}|_{\mathbb{R}^{n}}^{1 / 2}+\left|b_{j 1}^{s}\right||y-\bar{y}|_{\mathbb{R}^{n}} \frac{\beta\left(E|y-\bar{y}|_{\mathbb{R}^{n}}^{1 / 4}\right.}{\delta^{2}} \\
\leq & y_{s}-\bar{y}_{s}-3|y-\bar{y}|_{\mathbb{R}^{n}}+\mathcal{B}_{M}\left(\frac{1}{\mathcal{B}_{M}}\right)|y-\bar{y}|_{\mathbb{R}^{n}} \leq-|y-\bar{y}|_{\mathbb{R}^{n}}<0 .
\end{aligned}
$$

This leads to a contradiction; indeed if $y_{s}>\bar{y}_{s}$, then $y_{s}(\cdot)$ and $\bar{y}_{s}(\cdot)$ are solutions of the same Cauchy problem

$$
\dot{y}_{s}(r)=\frac{1}{2} \sum_{i=2}^{m} x_{i} b_{j i}^{s}+b_{j 1}^{s} \psi\left(x_{2}, \ldots, x_{j-1}, r, x_{j+1}, \ldots, x_{m}, y_{1}, \ldots, y_{s-1}, y_{s}(r), y_{s+1}, \ldots, y_{n}\right)
$$

with initial data $y_{s}\left(x_{j}\right)=y_{s}$ and $\bar{y}_{s}$ respectively, but two such solutions cannot meet, while $y_{s}\left(x_{j}\right)-$ $\bar{y}_{s}\left(x_{j}\right)>0$ and $y_{s}(t)-\bar{y}_{s}(t)<0$ for a certain $t \in\left(x_{j}-r_{0}, x_{j}+r_{0}\right)$ with $r_{0}$ sufficiently large.

On the other hand if $y_{s}=\bar{y}_{s}$, by (48) we conclude that $y_{s}(t) \neq \bar{y}_{s}(t)$ for $t=x_{j}+\frac{|y-\bar{y}|_{\mathbb{R} n}^{1 / 2}}{\delta}$ or

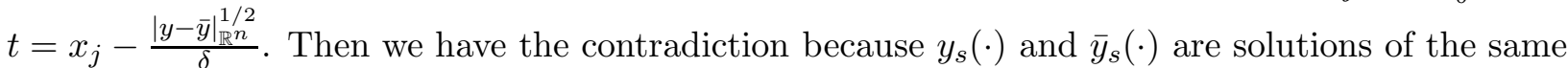
Cauchy problem (49) with initial data $y_{s}(t)$ and $\bar{y}_{s}(t)$ but two such solutions cannot meet, while $y_{s}=\bar{y}_{s}$.

Hence (45) follows.

Step 3. Now let $A, A^{\prime}, B \in \mathcal{I}$ (up to reducing $\mathcal{I}$ ) with $A=(x, y), A^{\prime}=\left(x^{\prime}, y^{\prime}\right)$ and $B=\left(x, y^{\prime}\right)$. We want to show that

$$
\frac{\left|\psi(B)-\psi\left(A^{\prime}\right)\right|}{\left|x-x^{\prime}\right|_{\mathbb{R}^{m-1}}^{1 / 2}} \leq N\left|A-A^{\prime}\right|_{\mathbb{R}^{m+n-1}}^{1 / 2}+\frac{3 h \delta}{\mathcal{B}_{m}}
$$

where $\delta=\delta\left(h^{2}\left|x-x^{\prime}\right|_{\mathbb{R}^{m-1}}\right)$. We suppose on the contrary that

$$
\frac{\left|\psi(B)-\psi\left(A^{\prime}\right)\right|}{\left|x-x^{\prime}\right|_{\mathbb{R}^{m-1}}^{1 / 2}}>N\left|A-A^{\prime}\right|_{\mathbb{R}^{m+n-1}}^{1 / 2}+\frac{3 h \delta}{\mathcal{B}_{m}} .
$$

Set

$$
D_{j}:=\gamma_{D_{j-1}}^{j}\left(x_{j}\right) \quad \text { for } j=2, \ldots, m
$$

with $D_{1}:=A^{\prime}$. A computation gives that

$$
D_{j}=\left(x_{2}, \ldots, x_{j}, x_{j+1}^{\prime}, \ldots, x_{m}^{\prime}, y^{D_{j}}\right)
$$

with

$$
y_{s}^{D_{j}}=y_{s}^{\prime}+\sum_{l=2}^{j}\left(b_{l 1}^{s} \int_{x_{l}^{\prime}}^{x_{l}} \psi\left(\gamma_{D_{j-1}}^{j}(r)\right) d r+\frac{1}{2}\left(x_{l}-x_{l}^{\prime}\right)\left(\sum_{i=2}^{l} x_{i} b_{l i}^{s}+\sum_{i=l+1}^{m} x_{i}^{\prime} b_{l i}^{s}\right)\right)
$$

for $s=1, \ldots, n$ and consequently, recalling that $h=\left(n \mathcal{B}_{M}(K+M)\right)^{1 / 2}$

$$
\left|y^{\prime}-y^{D_{m}}\right|_{\mathbb{R}^{n}} \leq n \mathcal{B}_{M}(M+K)\left|x-x^{\prime}\right|_{\mathbb{R}^{m-1}}=h^{2}\left|x-x^{\prime}\right|_{\mathbb{R}^{m-1}} .
$$

Moreover we have

$$
\sum_{j=2}^{m}\left|\psi\left(D_{j-1}\right)-\psi\left(D_{j}\right)\right|=\sum_{j=2}^{m}\left|\int_{x_{j}^{\prime}}^{x_{j}} w_{j}\left(\gamma_{D_{j-1}}^{j}(t)\right) d t\right| \leq N\left|x-x^{\prime}\right|_{\mathbb{R}^{m-1}}
$$


In order to stay in the $\mathcal{I}$ of the previous step, we want $x^{\prime}-x$ sufficiently small (and precisely when $\left.N\left|x-x^{\prime}\right|_{\mathbb{R}^{m-1}}^{1 / 2} \leq\left|x-x^{\prime}\right|_{\mathbb{R}^{m-1}}^{1 / 4} \leq \delta\right)$. By (151) and (52), we get

$$
\begin{aligned}
\left|\psi(B)-\psi\left(D_{m}\right)\right| & \geq\left|\psi(B)-\psi\left(A^{\prime}\right)\right|-\sum_{l=2}^{m}\left|\psi\left(D_{l-1}\right)-\psi\left(D_{l}\right)\right| \\
& >\left(N\left|A-A^{\prime}\right|_{\mathbb{R}^{m+n-1}}^{1 / 2}+\frac{3 h}{\mathcal{B}_{m}} \delta-N\left|x-x^{\prime}\right|_{\mathbb{R}^{m-1}}^{1 / 2}\right)\left|x-x^{\prime}\right|_{\mathbb{R}^{m-1}}^{1 / 2} \\
& \geq \frac{3 h}{\mathcal{B}_{m}} \delta\left|x-x^{\prime}\right|_{\mathbb{R}^{m-1}}^{1 / 2} \\
& \geq \frac{3}{\mathcal{B}_{m}} \delta\left|y^{\prime}-y^{D_{m}}\right|_{\mathbb{R}^{n}}^{1 / 2}
\end{aligned}
$$

so that we are in the first case again (see (45)) with the couple $B=\left(x, y^{\prime}\right), D_{m}=\left(x, y^{D_{m}}\right)$ instead $A, B$ respectively) which we have seen is not possible. Hence (50) holds.

Step 4. Using Step 2. and Step 3. we deduce that

$$
\begin{aligned}
\frac{\left|\psi(A)-\psi\left(A^{\prime}\right)\right|}{\left|A-A^{\prime}\right|_{\mathbb{R}^{m+n-1}}^{1 / 2}} & \leq \frac{|\psi(A)-\psi(B)|}{\left|y-y^{\prime}\right|_{\mathbb{R}^{n}}^{1 / 2}}+\frac{\left|\psi(B)-\psi\left(A^{\prime}\right)\right|}{\left|x-x^{\prime}\right|_{\mathbb{R}^{m-1}}^{1 / 2}} \\
& \leq \frac{3(1+h)}{\mathcal{B}_{m}} \delta\left(\max \left\{1, h^{2}\right\}\left|A-A^{\prime}\right|_{\mathbb{R}^{m+n-1}}\right)+N\left|A-A^{\prime}\right|_{\mathbb{R}^{m+n-1}}^{1 / 2} \\
& =\alpha\left(\left|A-A^{\prime}\right|_{\mathbb{R}^{m+n-1}}\right)
\end{aligned}
$$

for all $A=(x, y), A^{\prime}=\left(x^{\prime}, y^{\prime}\right), B=\left(x, y^{\prime}\right) \in \mathcal{I}$. Then according to (41) and (42) we have that $\lim _{r \rightarrow 0} \alpha(r)=0$ and we are able to control $\alpha$ with only $K, M, N, \mathcal{B}_{M}, \mathcal{B}_{m}$ and $\beta$.

\section{REFERENCES}

[1] L.Ambrosio, B.Kirchheim, Rectifiable sets in metric and Banach spaces, Math. Ann. 318 (2000), no. 3, 527-555.

[2] L.Ambrosio, F. Serra Cassano, D. Vittone, Intrinsic regular hypersurfaces in Heisenberg groups, J. Geom. Anal. 16, (2006), 187-232.

[3] G.Arena, R.Serapioni, Intrinsic regular submanifolds in Heisenberg groups are differentiable graphs, Calc. Var. Partial Differential Equations 35, (2009), no. 4, 517-536.

[4] F.Bigolin, L.Caravenna, F.Serra Cassano, Intrinsic Lipschitz graphs in Heisenberg groups and continuous solutions of a balance equation, Ann. I.H. Poincaré -AN (2014).

[5] F.Bigolin, F.Serra Cassano, Intrinsic regular graphs in Heisenberg groups vs. weak solutions of non linear firstorder PDEs, Adv. Calc. Var. 3, (2010), 69-97.

[6] A.Bonfiglioli, E.Lanconelli, F.Uguzzoni, Stratified Lie Groups and Potential Theory for their Sub-Laplacians, Springer Monographs in Mathematics, Springer-Verlag Berlin Heidelberg, New York (2007).

[7] D.Di Donato, Intrinsic differentiability and Intrinsic Regular Surfaces in Carnot groups, PhD Thesis, University of Trento, (2017).

[8] L.C.Evans, R.F.Gariepy, Measure Theory and Fine Properties of functions, CRC Press, BocaRaton, (1992).

[9] H.Federer, Geometric Measure Theory, Springer, (1969).

[10] B.Franchi, M.Marchi, R.Serapioni, Differentiability and Approximate Differentiability for intrinsic Lipschitz functions in Carnot groups and a Rademarcher Theorem, Anal. Geom. Metr. Spaces 2, (2014), 258-281.

[11] B.Franchi, R.Serapioni, Intrinsic Lipschitz Graphs within Carnot groups, The Journal of Geometric Analysis, Vol. 26 (3) (2016), 1946-1994.

[12] B.Franchi, R.Serapioni, F.Serra Cassano, Rectifiability and perimeter in the Heisenberg group, Math. Ann. 321 (2001), 479-531.

[13] B.Franchi, R.Serapioni, F.Serra Cassano, Regular hypersurfaces, Intrinsic Perimeter and Implicit Function Theorem in Carnot Groups, Comm. Anal. Geom. 11 (2003), no. 5, 909-944.

[14] B.Franchi, R.Serapioni, F.Serra Cassano, Regular Submanifolds, Graphs and area formula in Heisenberg Groups, Advances in Math. 211 (2007), no. 1, 152-203.

[15] B.Franchi, R.Serapioni, F.Serra Cassano, On the Structure of Finite Perimeter Sets in Step 2 Carnot Groups, The Journal of Geometric Analysis 13 (2003), no. 3, 421-466. 
[16] N.Garofalo, D.M.Nhieu, Isoperimetric and Sobolev inequalities for Carnot-Carathéodory spaces and the existence of minimal surfaces, Comm. Pure Appl. Math. 49 (1996), 1081-1144.

[17] B.Kirchheim, F.Serra Cassano, Rectifiability and parametrization of intrinsic regular surfaces in the Heisenberg group, Ann. Scuola Norm. Sup. Pisa Cl. Sci. (5) III, (2004), 871-896.

[18] A.Kozhevnikov, Propriétés métriques des ensembles de niveau des applications différentiables sur les groupes de Carnot, PhD Thesis, Université Paris-Sud, (2015).

[19] E.Le Donne, A Primer on Carnot Groups: Homogenous Groups, Carnot-Carathéodory Spaces, and Regularity of Their Isometries, Anal. Geom. Metr. Spaces, (2017), 5: 116-137.

[20] G.Lu, Embedding theorems into Lipschitz and BMO spaces and applications to quasilinear sub elliptic differential equations, Publ. Mat. 40 (1996), 301-329.

[21] V.Magnani, Elements of Geometric Measure Theory on Sub-Riemannian Groups, PhD Thesis, Scuola Normale Superiore, Edizioni della Normale, Pisa, (2002).

[22] V.Magnani, Towards Differential Calculus in Stratified Groups, J. Aust. Math. Soc. 95, n.1, (2013), 76-128.

[23] P.Mattila, Geometry of Sets and Measures in Euclidean Spaces, Cambridge University Press, Cambridge, (1995).

[24] J.Mitchell, On Carnot-Carathéodory metrics, J. Differ. Geom. 21, (1985), 35-45.

[25] P.Pansu, Métriques de Carnot-Carathéodory et quasiisométries des espaces symétriques de rang un, Ann. of Math., 129, (1989), 1-60.

[26] R.Serapioni, Intrinsic difference quotients, Harmonic Analysis, Partial Differential Equations and Applications, Birkhäuser (2017), 165-192.

[27] F.Serra Cassano, Some topics of geometric measure theory in Carnot groups, Geometry, Analysis and Dynamics on sub-Riemannian Manifolds, Vol. I, European Mathematical Society (2016), 1-121.

[28] D.Vittone, Submanifolds in Carnot Groups, PhD Thesis, Scuola Normale Superiore, Edizioni della Normale, Pisa, (2008).

Daniela Di Donato: Dipartimento di Matematica, Università di Trento, Via Sommarive 14, 38123, Povo (Trento) - ItAly,

E-mail address: daniela.didonato@unitn.it 